\title{
The Meaning of Negative Premises in Transition System Specifications
}

\author{
ROLAND BOL \\ Uppsala University, Uppsala, Sweden
}

\begin{abstract}
AND
JAN FRISO GROOTE

Centre for Mathematics and Computer Science, Amsterdam, The Netherlands
\end{abstract}

\begin{abstract}
We present a general theory for the use of negative premises in the rules of Transition System Specifications (TSSs). We formulate a criterion that should be satisfied by a TSS in order to be meaningful, that is, to unequivocally define a transition relation. We also provide powerful techniques for proving that a TSS satisfies this criterion, meanwhile constructing this transition relation. Both the criterion and the techniques originate from logic programming [van Gelder et al. 1988; Gelfond and Lifschitz 1988] to which TSSs are close. In an appendix we provide an extensive comparison between them.

As in Groote [1993], we show that the bisimulation relation induced by a TSS is a congruence, provided that it is in ntyft/ntyxt-format and can be proved meaningful using our techniques. We also considerably extend the conservativity theorems of Groote [1993] and Groote and Vaandrager [1992]. As a running example, we study the combined addition of priorities and abstraction to Basic Process Algebra (BPA). Under some reasonable conditions we show that this TSS is indeed meaningful, which could not be shown by other methods [Bloom et al. 1995; Groote 1993]. Finally, we provide a sound and complete axiomatization for this example.
\end{abstract}

Categories and Subject Descriptors: D.3.1 [Programming Languages]: Formal Definitions and Theory; F.3.1 [Logics and Meanings of Programs]: Specifying and Verifying and Reasoning about Programs; F.3.2 [Logics and Meanings of Programs]: Semantics of Programming Languages; 1.2.3 [Artificial Intelligence]: Deduction and Theorem Proving.

General Terms: Algebra, Semantics

Additional Key Words and Phrases: Bisimulation, congruence, conservative extension of TSSs, logic programming, negative premises, ntyft/ntyxt-format, priorities and abstraction, process algebra,

R. Bol was partly supported by the European Communities under ESPIRIT Basic Research Action 3020 (Integration).

J. F. Groote was supported by the European Communities under RACE project no. 1046 (SPECS) and ESPIRIT Basic Research Action 3006 (CONCUR).

Authors' present addresses: R. Bol, Department of Computer Systems, Uppsala University, P.O. Box 325, S-751-05 Uppsala, Sweden, e-mail: rolandb@docs.uu.se; J. F. Groote, Centre for Mathematics and Computer Science, P.O. Box 4079, 1000 AB Amsterdam, The Netherlands, e-mail: jfg@cwi.al.

Permission to make digital/hard copy of part or all of this work for personal or classroom use is granted without fee provided that the copies are not made or distributed for profit or commercial advantage, the copyright notice, the title of the publication, and its date appear, and notice is given that copying is by permission of the Association for Computing Machinery (ACM), Inc. To copy otherwise, to republish, to post on servers, or to redistribute to lists, requires prior specific permission and/or a fee.

(c) 1996 ACM 0004-5411/96/0900-0863 $\$ 03.50$ 
stable model semantics, stratification, structured operational semantics (SOS), sum of TSSs, transition relation, transition system specification (TSS), well founded model semantics

\section{Introduction}

Since its introduction in Hennessy and Plotkin [1979] and Plotkin [1981], Plotkin-style operational semantics has become a popular means for giving meaning to process calculi, specification and programming languages in terms of transition systems. A transition system consists mainly of a transition relation which is specified by a set of rules forming a Transition System Specification (TSS) [Groote and Vaandrager 1992]. Recently, the use of negative premises in these rules has become popular ${ }^{1}$ because it allows one to define more operators in an easy way. However, the logical meaning of those negative premises is not always clear. Therefore, the formal foundation of some of these articles is somewhat questionable. The problematic nature of negative premises has already been observed in Baeten and Bergstra [1988], Bloom et al. [1995], and Groote [1993].

In this paper, we provide a way to treat negative premises in general and we study some of the consequences of this treatment. The fundamental problem of negative premises in TSSs is that they cannot be proved in the same way positive premises can. In order to overcome this problem, we resort to a non-classical treatment of negation, similar to default logic [Reiter 1980] and logic programming [Gelfond and Lifschitz 1988]. Without negative premises the notion of proof is standard. With negative premises we may only use the rules for which the negative premises hold. A negative premise holds by default, that is unless the opposite can be proved. Now suppose $\rightarrow$ contains all transitions that can be proved in this way. Then it must satisfy:

$\rightarrow$ is the set of transitions that are provable by those rules of which the negative premises are consistent with $\rightarrow$.

Following Gelfond and Lifschitz [1988], we call such a transition relation stable for the TSS.

It is possible that a TSS has zero, one, or more stable transition relations. If a TSS $P$ has exactly one stable transition relation then we propose that this relation is the transition relation associated with $P$. If a TSS has zero or more than one stable transition relation, it is hard to imagine that any specific transition relation can be associated with it on reasonable grounds. That is, unless one is prepared to associate with a TSS a transition relation that is not decisive about all transitions. But this is not considered appropriate for the field of operational semantics.

In general, it is difficult to show that a TSS has a unique stable transition relation. However, some techniques have been developed for showing its existence. The first technique, called stratification, is presented in Groote [1993]. It is based on the notion of local stratification in logic programming [Przymusinski 1987]. In this article we show that the transition relation associated with a

\footnotetext{
${ }^{1}$ See Baeten and Bergstra [1986], Bloom et al. [1995], Bolognesi et al. [1990], Groote [1993], Hennessy and Regan [1990], Ichikawa et al. [1990], Janicki [1987], Langerak [1989], Nicollin et al. [1990], and Pneuli [1985].
} 
stratified TSS according to Groote [1993] is indeed the unique stable transition relation for it. This also implies the same fact for positive TSSs and TSSs in the so-called GSOS-format [Bloom et al. 1995], as they are stratified.

Stratification is an intuitively appealing technique, and quite easy to use, but it is not always strong enough. Here, we introduce a more powerful technique, based on well-founded models in logic programming [van Gelder et al. 1988; Przymusinski 1989]. This technique, which we call reduction, is more powerful than stratification, but also more difficult to use. The two techniques can be amalgamated, using reduction when necessary and stratification when possible. This is demonstrated in our running example, showing that under some reasonable conditions a transition relation can be associated with it.

A desirable property for a TSS is that the strong bisimulation equivalence induced by it [Milner 1980; Park 1981] is a congruence. In Groote and Vaandrager [1992] the tyft/tyxt-format was introduced, as a syntactical condition on TSSs that guarantees this property for positive TSSs. In Groote [1993], this condition was generalized to the ntyft/ntyxt-format for stratified TSSs. Here we show that the same condition is sufficient for all TSSs for which the reduction technique works. In contrast, we show that the condition is not sufficient for TSSs having an associated transition relation that is not produced by reduction.

It can be useful to enrich a given language with additional language constructs. In order to do this in a systematic way, the sum of two TSSs has been introduced [Groote and Vaandrager 1992]. The sum of two TSSs $P_{0}$ and $P_{1}$ is called a conservative extension of $P_{0}$ if certain relevant properties of terms over the signature of $P_{0}$ are preserved. In Groote [1993], syntactical conditions on stratified TSSs were given ensuring that their sum is conservative. Here we generalize these conditions considerably and we extend them to TSSs for which the reduction technique works.

Throughout the paper, we use an example to illustrate these techniques: a TSS specifying the operational semantics of Basic Process Algebra (BPA) extended with priorities [Baeten et al. 1986] and abstraction [van Glabbeek 1987; Milner 1980]. We show using reduction and stratification that this TSS is meaningful. In Section 10 we give a sound and complete axiomatization of strong bisimulation equivalence induced by this TSS. It turns out that most of the standard techniques for positive TSSs can still be used.

1.1. Related Work. For those readers who are interested in reading more about TSSs, we give a short summary of recent results in this area. With regard to negative premises in TSSs the reader is referred to van Glabbeek [1995], which provides an neat overview of potential semantics for negative premises in rules, including an alternative formulation of our notion of TSSs that are positive after reduction. In van Glabbeek [1995], such TSSs are called (ws-)complete. An argument is given why a TSS with a unique stable model but which is not (ws-)complete should not be considered as meaningful.

It is interesting to note that Fokkink and van Glabbeek [1995] have shown that the well-foundedness condition on TSSs in order to show the Congruence Theorem to hold (Theorem 8.14) can be omitted. Their main argument is that for every ws-complete TSS in ntyft/ntyxt format an equivalent well-founded ws-complete TSS in the same format can be constructed. 
There appear two main streams in extending the format of operational rules. In the first one, explicit predicates are allowed in rules [Baeten and Verhoef 1993; Verhoef 1995]. It is shown that for the path and panth formats the Congruence Theorem for bisimulation still holds. In Fokkink and Verhoef [1995] and Howe [1996], transition system specifications that allow substitutions are being studied. In Fokkink and Verhoef [1995] and Verhoef [1994], sufficient criteria are provided to guarantee conservativity of extensions (cf. Theorem 9.5). In Howe [1996], structured evaluation systems are introduced, and it is proven that bisimulation is a congruence for the operators defined using such systems.

In Aceto et al. [1994], it is shown how to generate automatically a complete axiom system for bisimulation semantics given a Transition System Specification where the rules are in a certain format. In Aceto [1994], this format is somewhat relaxed but such that still finitary sets of axioms are generated. In Bloom [1995], the issue of generating an axiom system is also shortly touched upon.

In the category of miscellaneous results, we find [D'Argenio 1995] who shows a conservativity result for preorders. In Bloom [1995] and van Glabbeek [1987], formats for observation equivalences are being studied. In Aceto and Ingólfsdóttir [1994/1995], denotational models for languages specified using particular types of rules are given.

This paper is structured as follows:

1. Introduction

2. Preliminaries

Transition relations, TSSs and the running example are introduced.

3. Transition relations for TSSs

Several proposals for associating a transition relation with a TSS are given and stratified TSSs are defined. It is concluded that none of the existing techniques can handle the running example.

4. TSSs and their associated transition relations

We identify meaningful TSSs using stable transition relations and 'stripping' of TSSs.

5. Reducing TSSs

The reduction technique is presented and its soundness is proved.

6. Reduction and stratification

Reduction is stronger than stratification. An amalgamation of both techniques provides a meaning to the running example.

7. Bisimulation relations

$P$-bisimulation and $P \Rightarrow Q$-bisimulation are introduced.

8. The ntyft/ntyxt-format and the Congruence Theorem

The Congruence Theorem holds for TSSs in ntyft/ntyxt-format for which reduction works.

9. Conservative extensions of TSSs

The sum of TSSs is conservative under some liberal conditions.

10. An axiomatization of priorities with abstraction

A sound and complete axiomatization for the running example is given. 
Appendix A. The relation between TSSs and logic programs.

We provide an extensive comparison between TSSs and logic programs.

\section{Preliminaries}

In this section we provide the basic concepts of this paper: transition relations and Transition System Specifications (TSSs). An example of a TSS is given in which priorities and abstraction are integrated in BPA. This example will serve as a running example throughout this article.

We assume the presence of an infinite set $V$ of variables with typical elements $x, y, z \ldots$.

Definition 2.1. A (single sorted) signature is a structure $\Sigma=(F$, rank) where: $-F$ is a set of function names disjoint with $V$,

- rank: $F \rightarrow \mathbf{N}$ is a rank function which gives the arity of a function name; if $f \in F$ and $\operatorname{rank}(f)=0$ then $f$ is called a constant name.

Let $W \subseteq V$ be a set of variables. The set of $\Sigma$-terms over $W$, notation $T(\Sigma, W)$, is the least set satisfying:

$-W \subseteq T(\Sigma, W)$,

-if $f \in F$ and $t_{1}, \ldots, t_{\operatorname{rank}(f)} \in T(\Sigma, W)$, then $f\left(t_{1}, \ldots, t_{\operatorname{rank}(f)}\right) \in T(\Sigma, W)$.

$T(\Sigma, \emptyset)$ is abbreviated by $T(\Sigma)$; elements from $T(\Sigma)$ are called closed or ground terms. $T(\Sigma)$ is used to abbreviate $T(\Sigma, V)$, the set of open terms. Clearly, $T(\Sigma) \subset$ $\mathbb{T}(\Sigma)$. $\operatorname{Var}(t) \subseteq V$ is the set of variables in a term $t \in \mathbb{T}(\Sigma)$. A substitution $\sigma$ is a mapping in $V \rightarrow \mathbb{T}(\Sigma)$. A substitution $\sigma$ is extended to a mapping $\sigma: \mathbb{T}(\Sigma) \rightarrow$ $\mathbb{T}(\Sigma)$ in a standard way by the following definition:

$-\sigma\left(f\left(t_{1}, \ldots, t_{\operatorname{rank}(f)}\right)\right)=f\left(\sigma\left(t_{1}\right), \ldots, \sigma\left(t_{\operatorname{rank}(f)}\right)\right)$ for $f \in F$ and $t_{1}, \ldots$, $t_{\text {rank }(f)} \in \mathbb{T}(\Sigma)$.

A substitution is closed (or ground) if it maps all variables onto closed terms.

A transition relation prescribes what activities, represented by labeled transitions, can be performed by terms over some signature. Generally, the signature represents a programming or a specification language and the terms are programs. The transition relation models the operational behavior of these terms.

Definition 2.2. Let $\Sigma$ be a signature and $A$ a set of labels. A (labeled) transition relation is a subset $\rightarrow$ of $\operatorname{Tr}(\Sigma, A)$, where $\operatorname{Tr}(\Sigma, A)=T(\Sigma) \times A \times$ $T(\Sigma)$. Elements $\left(t, a, t^{\prime}\right)$ of a transition relation are written as $t \stackrel{a}{\rightarrow} t^{\prime}$.

A transition relation is often defined by means of a Transition System Specification (TSS). Plotkin [Hennessy and Plotkin 1979; Plotkin 1981] defended the use of TSSs to give an operational semantics, and therefore a TSS is sometimes called an operational semantics in Plotkin style. The term, TSS, was first coined in Groote and Vaandrager [1992] for a system in which rules had only positive premises. Negative premises were added in Groote [1993].

Definition 2.3. A TSS (Transition System Specification) is a triple $P=$ $(\Sigma, A, R)$ with $\Sigma$ a signature, $A$ a set of labels and $R$ a set of rules of the form:

$$
\frac{\left\{t_{k} \stackrel{a_{k}}{\rightarrow} t_{k}^{\prime} \mid k \in K\right\} \cup\left\{t_{l} \stackrel{b_{l}}{\rightarrow} \mid l \in L\right\}}{t \stackrel{a}{\rightarrow} t^{\prime}}
$$


with $K, L$ (possibly infinite) index sets, $t_{k}, t_{k}^{\prime}, t_{l}, t, t^{\prime} \in \mathbb{T}(\Sigma), a_{k}, b_{l}, a \in$ $A(k \in K, l \in L)$. An expression of the form $t \stackrel{a}{\rightarrow} t^{\prime}$ is called a (positive) literal. $t \rightarrow$ is called a negative literal. $\varphi, \psi, \chi$ are used to range over literals. For a literal $\psi$, source $(\psi)$ denotes the term at the left hand side of $\psi$ and, if $\psi$ is positive, target $(\psi)$ denotes the term at the right hand side. For any rule $r \in R$ the literals above the line are called the premises of $r$, notation prem $(r)$, and the literal below the line is called the conclusion of $r$, denoted as conc $(r)$. Furthermore, we write pprem $(r)$ for the set of positive premises of $r$ and nprem $(r)$ for the set of negative premises of $r$. A rule $r$ is called positive if there are no negative premises, that is, $n$ prem $(r)=\emptyset$. A TSS is called positive if it has only positive rules. A rule is called an axiom if its set of premises is empty. An axiom $\frac{\emptyset}{t \rightarrow a} t^{\prime}$ is often written as $t \stackrel{a}{\rightarrow} t$ '. The notions 'substitution', 'Var' and 'closed' extend to literals and rules as expected.

Throughout this paper, we use the following Transition System Specification scheme to illustrate the techniques we introduce. It describes the semantics of a small basic process language extended with priorities and abstraction. This combination has not been studied before due to the technical complications that are involved. Priorities are investigated in Baeten et al. [1986], Best and Koutny [1990], Camilleri [1989], and Cleaveland and Hennessy [1988]. We follow the line set out by Baeten et al. [1985] who introduced a priority operator $\theta$. We base our example on $\mathrm{BPA}_{\delta \epsilon \tau}$, Basic Process Algebra with $\tau, \epsilon$, and $\delta$ as introduced in Groote and Vaandrager [1992], and extend it with recursion and priorities. For a general overview of process algebra, the reader is referred to Baeten and Weijland [1990].

Example 2.4 (BPA $_{\delta \epsilon \tau}$ with priorities). We assume that we have a given set $A c t$ of actions that represent the basic activities that can be performed by processes. $A c t_{\tau}=A c t \cup\{\tau\}$ is a set of actions containing the symbol $\tau$ representing internal or hidden activity. Moreover, we assume a partial ordering $<$ on $A c t_{\tau}$, which we call the priority relation: actions higher in the ordering have a higher priority than actions lower in the ordering. We assume that $<$ is backwardly well-founded, that is, the inverse of $<$ constitutes a well-founded ordering.

Our signature contains a constant $a$ for each action $a \in A c t_{\tau}$. Moreover, we have two special constants $\delta$ and $\epsilon . \delta$ is called inaction (or deadlock) and it represents the process that cannot do anything at all. In particular, $\delta$ cannot terminate. $\epsilon$ is called the empty process, which cannot do anything but terminate.

Two basic operators compose smaller into larger processes: sequential composition is written as '" and alternative composition is denoted by + . We often leave out the '.' and assume that '.' binds stronger than + .

Actions can be abstracted away: for all $I \subseteq A c t$ the unary abstraction operator $\tau_{I}$ performs this task by renaming all actions in $I$ to $\tau$.

For recursion it is assumed that there is some given set $\Xi$ of process names. Each process name $X \in \Xi$ is treated as a constant in the signature. Furthermore, we assume that a set $E$ of process declarations is given. For each process name $X$ in $\Xi$ there is a declaration $X \Leftarrow t_{X} \in E$ where $t_{X}$ is a closed term over the signature. Terms that do not contain process names are called recursion free.

The remaining operators in the signature deal with priorities. The priority operator $\theta$ acts as a sieve: $\theta(x)$ only allows those actions from $x$ that have highest 


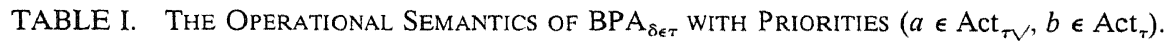

\begin{tabular}{|c|c|c|c|c|}
\hline$\epsilon:$ & R1: & $\epsilon \stackrel{\vee}{\longrightarrow} \delta$ & & \\
\hline$a:$ & $\mathrm{R} 2:$ & $a \stackrel{a}{\longrightarrow} \epsilon$ if $a \in A c t_{\tau}$ & & \\
\hline \multirow{2}{*}{$+:$} & \multirow{2}{*}{ R3.1: } & $x \stackrel{a}{\longrightarrow} x^{\prime}$ & \multirow{2}{*}{ R3.2: } & $y \stackrel{a}{\longrightarrow} y^{\prime}$ \\
\hline & & $x+y \stackrel{a}{\rightarrow} x^{\prime}$ & & $\overline{x+y \stackrel{a}{\longrightarrow} y^{\prime}}$ \\
\hline \multirow{2}{*}{$\cdot:$} & \multirow{2}{*}{ R4.1: } & $\stackrel{x}{\longrightarrow} x^{\prime}$ if $a \in A c t_{\tau}$ & \multirow{2}{*}{ R4.2: } & $\stackrel{\vee^{\prime}}{\longrightarrow} x^{\prime} \quad y \stackrel{a}{\longrightarrow} y^{\prime}$ \\
\hline & & $x \cdot y \stackrel{a}{\longrightarrow} x^{\prime} \cdot y$ & & $x \cdot y \stackrel{a}{\longrightarrow} y^{\prime}$ \\
\hline \multirow{2}{*}{$\theta:$} & \multirow{2}{*}{ R5.1: } & 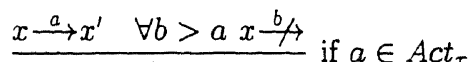 & \multirow{2}{*}{ R5.2: } & $\stackrel{\sqrt{ }}{\rightarrow} x^{\prime}$ \\
\hline & & $\theta(x) \stackrel{a}{\longrightarrow} \theta\left(x^{\prime}\right)$ & & $\theta(x) \stackrel{\sqrt{ }}{\longrightarrow} \theta\left(x^{\prime}\right)$ \\
\hline \multirow{2}{*}{$\triangleleft:$} & \multirow{2}{*}{ R6.1: } & 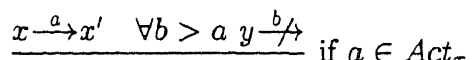 & \multirow{2}{*}{ R6.2: } & $x \stackrel{\vee}{\longrightarrow} x^{\prime}$ \\
\hline & & $x \triangleleft y \stackrel{a}{\longrightarrow} x^{\prime}$ & & $\overline{x \triangleleft y \stackrel{\sqrt{ }}{\rightarrow} x^{\prime}}$ \\
\hline \multirow{2}{*}{$\tau_{I}:$} & \multirow{2}{*}{ R7.1: } & $\stackrel{x^{a}}{\longrightarrow} x^{\prime}$ if $a \notin I$ & \multirow{2}{*}{ R7.2: } & $x \stackrel{a}{\longrightarrow} x^{\prime}$ \\
\hline & & $\tau_{I}(x) \stackrel{a}{\longrightarrow} \tau_{I}\left(x^{\prime}\right)$ & & $\tau_{I}(x) \stackrel{\tau}{\longrightarrow} \tau_{I}\left(x^{\prime}\right)$ \\
\hline \multirow[t]{3}{*}{ recursion: } & R8: & $\frac{t_{X \stackrel{a}{\longrightarrow}} y}{X \stackrel{a}{\longrightarrow} y}$ if $X \Leftarrow t_{X} \in E$ & & \\
\hline & R9.1: & $a \stackrel{a}{\longrightarrow} \tau$ if $a \in A c t_{\tau}$ & & \\
\hline & R9.2: & $\frac{x \stackrel{\tau}{\longrightarrow} y \quad y \stackrel{a}{\longrightarrow} z}{x \stackrel{a}{\longrightarrow} z}$ & R9.3: & $\frac{x \stackrel{a}{\longrightarrow} y \quad y \stackrel{\tau}{\longrightarrow} z}{x \stackrel{a}{\longrightarrow} z}$ \\
\hline
\end{tabular}

priority. For the axiomatization of $\mathrm{BPA}_{\delta \epsilon \tau}$ with priorities, which is given in Section 10, we need the unless operator $\triangleleft$, which was introduced in Baeten et al. [1986]. This operator is applied on two operands and only allows an action in its left-hand side provided the right-hand side cannot do any action with higher priority.

When $\left(A c t_{\tau},<\right)$ and $(\Xi, E)$ are fixed, we obtain a TSS which is an instance of $\mathrm{BPA}_{\delta \epsilon \tau}$ with priorities. Such an instance will be denoted as $P_{\theta}=\left(\Sigma_{\theta}, A_{\theta}, R_{\theta}\right)$. The signature $\Sigma_{\theta}=\left(F_{\theta}, r a n k_{\theta}\right)$ is described above. The labels in $A_{\theta}$ are exactly those in $A c t_{\tau}$ together with one special symbol $V$ which is used to signal termination. If a process term $t$ can perform a $\vee$-step, that is, $t \stackrel{\vee}{\rightarrow} t^{\prime}$, this means that $t$ has an option to terminate.

The rules in $R_{\theta}$ are given in Table I. Here, the action $a$ ranges over $A c t_{\tau \vee}=$ $A c t_{\tau} \cup\{\vee\}$ and $b$ ranges over $A c t_{\tau}$. In rules R5.1 and R6.1 we use the notation $\forall b>a x \stackrel{b}{\rightarrow}$ which means that for all $b$ with higher priority than $a$, there is a negative premise $x \stackrel{b}{\Rightarrow}$. Rule R5.1 is intuitively appealing. It says that $\theta(x)$ may do an $a$-action if $x$ can do this action and $x$ cannot do any action with higher priority. But there is a snag in it. Due to the negative premises, it is not at all straightforward to see that $P_{\theta}$ defines a transition relation. In fact, in Example 4.8 , we will present a case in which it does not make sense at all. 
Rules R9.1-R9.3 are explained below. We think that the remaining rules are self-explanatory, although we like to point out that rule R4.2 makes use of a process that explicitly signals termination.

Rules R9.1-R9.3 [van Glabbeek 1987] model the properties of $\tau$. R9.2 and R9.3 say that whenever an action $a$ is observed in some time interval, numerous unobservable $\tau$-actions can also happen during the same time, both before and after $a$. Rule R9.1 says that if an action $a$ is observed, some internal activity may exist before the next action can take place.

Through these rules, our interpretation of $\tau$ becomes observation equivalence as introduced by van Glabbeek [1987]. That is, two terms are observation equivalent in a transition system defined by a TSS if and only if the terms are strongly bisimilar (as defined in Section 7) in the transition system defined by the TSS augmented with rules R9.1-R9.3 (assuming, of course, that these TSSs indeed define transition relations).

Van Glabbeek's equivalence is a minor variant of the observation equivalence as introduced by Milner [1980]. The difference boils down to the rule KFAR [Baeten and Weijland 1990] which is valid in Milner's observation equivalence, whereas in van Glabbeek's only $\mathrm{KFAR}^{-}$holds [Baeten and Weijland 1990]. Both rules deal with divergence through the assumption of fair abstraction, but in a slightly different way. Without this modification, we cannot allow internal actions to have any priority relation with ordinary actions. In order to make this statement concrete, we must give a precise definition of the intuition "the priority of internal actions." Our intuition is that the priority operator $\theta$ should at least obey the following laws (also when $a$ or $b$ are $\tau$-actions):

$-\theta(a x)=a \theta(x)$, that is, $\theta$ distributes over sequential composition.

$-\theta(a x+b y)=\theta(a x)$ if $b<a$, that is, in a context where $a$ has a higher priority than $b, b$ can never be chosen over $a$.

The first of the following two examples shows that under these assumptions Milner's observation equivalence does not allow internal actions to have priority over ordinary actions. The second example shows that internal actions can also not have lower priority than ordinary actions. This second example does apply equally well to our setting and provides one of the reasons why we disallow visible actions to have priority over internal actions (cf. discussion before Theorem 6.6).

Example 2.5. Let $=$ denote observation equivalence in the sense of Milner, and let $\tau^{*}$ denote the process that can execute $\tau$ an arbitrary number of times (formally: $\tau_{\{i\}}(X)$, with $X \Leftarrow i \cdot X+\epsilon \in E$ ). According to KFAR, we have $a \tau^{*} b=a b$ and $a \tau^{*} \delta=a \delta$. Then $\tau>b$ leads to $a b=\theta(a b)=\theta\left(a \tau^{*} b\right)=$ $\theta\left(a \tau^{*} \delta\right)=\theta(a \delta)=a \delta$, which should not be valid in any reasonable equivalence.

Example 2.6. Consider the following consequence of the second tau law $\tau(a+b)=a+\tau(a+b)$ that is valid in both Milner's observation equivalence and van Glabbeek's equivalence. Assuming that $\tau$ has lower priority than $a$, and $a$ and $b$ are independent, we find $\tau(a+b)=\theta(\tau(a+b))=\theta(a+\tau(a+b))$ $=a$, which should not be valid in any reasonable equivalence. 


\section{Transition relations for TSSS}

We have introduced TSSs as a formalism for specifying transition relations. Thus, a most fundamental question is, which transition relation is actually defined by a TSS? In this section we outline some answers proposed in the literature for several classes of TSSs. Then we show that these techniques are not capable of handling our running example satisfactorily. In the next sections we show how to solve this problem.

As a first step, a link between the transitions in a transition relation and the literals in TSSs is established.

Definition 3.1. Let $\rightarrow$ be a transition relation. A positive ground literal $\psi$ holds in $\rightarrow$ or $\psi$ is valid in $\rightarrow$, notation $\rightarrow \vDash \psi$, if the transition $\psi \in \rightarrow$. A negative ground literal $t \stackrel{a}{\rightarrow}$ holds in $\rightarrow$, notation $\rightarrow \vDash t \stackrel{a}{\rightarrow}$, if for no $t^{\prime} \in T(\Sigma)$ the transition $t \stackrel{a}{\rightarrow} t^{\prime} \in \rightarrow$. For a set of ground literals $\Psi$, we write $\rightarrow \vDash \Psi$ iff $\forall \psi$ $\in \Psi: \rightarrow \vDash \psi$.

Remark 3.2. Suppose we have two transition relations $\rightarrow_{1}$ and $\rightarrow_{2}$ such that $\rightarrow_{1} \subseteq \rightarrow_{2}$. For any set of positive literals $\Psi$ it is clear that $\rightarrow_{1} \vDash \Psi$ implies $\rightarrow_{2} \vDash$ $\Psi$. However, if $\Psi$ is a set of negative literals, then $\rightarrow_{2} \vDash \Psi$ implies $\rightarrow_{1} \vDash \Psi$. We shall often use this kind of reasoning.

What is the transition relation defined by a TSS? At least one may require that a transition relation associated with a TSS $P$ obeys the rules of $P$, that is, if the premises of a ground instance of a rule in $P$ are valid in $\rightarrow$, then the conclusion is also valid in $\rightarrow$. (In terms of logic: the rules of $P$, interpreted as implications, are true in $\rightarrow$ ).

Definition 3.3. Let $P=(\Sigma, A, R)$ be a TSS and let $\rightarrow \subseteq \operatorname{Tr}(\Sigma, A)$ be a transition relation. $\rightarrow$ is a model of $P$ if:

$$
\psi \in \rightarrow \Leftarrow \exists r \in R \text { and } \exists \sigma: V \rightarrow T(\Sigma) \text { such that: }\left\{\begin{array}{l}
\rightarrow \vDash \operatorname{prem}(\sigma(r)) \text { and } \\
\operatorname{conc}(\sigma(r))=\psi .
\end{array}\right.
$$

On the other hand, a transition $\psi$ should not be incorporated in the transition relation $\rightarrow$ of a TSS $P$ unless there is a good reason to do so, namely a rule in $P$ with valid premises in $\rightarrow$ concluding $\psi$.

Definition 3.4. Let $P=(\Sigma, A, R)$ be a TSS. Let $\rightarrow \subseteq \operatorname{Tr}(\Sigma, A)$ be a transition relation. $\rightarrow$ is supported by $P$ if:

$$
\psi \in \rightarrow \Rightarrow \exists r \in R \text { and } \exists \sigma: V \rightarrow T(\Sigma) \text { such that: }\left\{\begin{array}{l}
\rightarrow \vDash \operatorname{prem}(\sigma(r)) \text { and } \\
\operatorname{conc}(\sigma(r))=\psi .
\end{array}\right.
$$

Combining the previous definitions, we get:

Definition 3.5. Let $P=(\Sigma, A, R)$ be a TSS. Let $\rightarrow \subseteq \operatorname{Tr}(\Sigma, A)$ be a transition relation. $\rightarrow$ is a supported model of $P$ if $\rightarrow$ is supported by $P$ and $\rightarrow$ is a model of $P$.

The notion of $\rightarrow$ being a supported model of $P$ was introduced in Bloom et al. [1995] as ' $\rightarrow$ agrees with $P$ '. Although the transition relation associated with a TSS should certainly be a supported model of it, the notion of supportedness is 
generally not sufficient to exclude all superfluous transitions from the transition relation. This is shown by the following example:

Example 3.6. Suppose we have a TSS $P$ with one constant $f$, one label $a$ and the following rules:

$$
\frac{f \stackrel{a}{\rightarrow} f}{f \stackrel{a}{\rightarrow} f} .
$$

We would like $P$ to define the transition relation $\rightarrow_{P}=\emptyset$. We feel that there is not enough reason to add $f \stackrel{a}{\rightarrow} f$ to $\rightarrow_{P}$ as it can only be 'derived' by assuming that it is already in $\rightarrow_{P}$.

However, both $\emptyset$ and $\{f \stackrel{a}{\rightarrow} f\}$ are supported models of $P$.

For positive TSSs this shortcoming is easily remedied by associating with a TSS $P$ the least transition relation (with respect to set inclusion) that is a model of $P$. The existence of this least model follows from the model intersection property stated below.

Lemma 3.7 (Model Intersection Property). Let $P$ be a positive TSS and let $\mathscr{C}$ be a collection of models of $P$. Then $\cap \mathscr{C}$ is a model of $P$.

PROof. Let $r$ be a ground instance of a rule of $P$. If $\cap \mathscr{\ell} \vDash \operatorname{prem}(r)$, then for every $\rightarrow \in \mathscr{C}: \rightarrow \vDash \operatorname{prem}(r)$, thus for every $\rightarrow \in \mathscr{b}: \rightarrow \vDash \operatorname{conc}(r)$, as $\mathscr{b}$ is a collection of models. Thus $\cap \mathscr{C} \vDash \operatorname{conc}(r)$.

Thus, we have the following definition:

Definition 3.8. The transition relation $\rightarrow_{P}$ associated with a positive TSS $P$ is the least model of $P$ with respect to set inclusion.

Traditionally [Groote and Vaandrager 1992; Hennessy and Plotkin 1979; Plotkin 1981], a different definition of the transition relation associated with a positive TSS was given, based on the provability of transitions. We show that these two characterizations are equivalent.

Definition 3.9. Let $P=(\Sigma, A, R)$ be a positive TSS. A proof of a positive literal $\psi$ from $P$ is a well-founded, upwardly branching tree of which the nodes are labeled by literals $t \stackrel{a}{\rightarrow} t^{\prime}$ with $t, t^{\prime} \in \mathbb{T}(\Sigma)$ and $a \in A$, such that:

-the root is labeled with $\psi$,

-if $\chi$ is the label of a node $q$ and $\left\{\chi_{i} \mid i \in I\right\}$ is the set of labels of the nodes directly above $q$, then there is a rule $\left\{\varphi_{i} \mid i \in I\right\} / \varphi$ in $R$ and a substitution $\sigma: V$ $\rightarrow \mathbb{T}(\Sigma)$ such that $\chi=\sigma(\varphi)$ and $\chi_{i}=\sigma\left(\varphi_{i}\right)$ for $i \in I$.

A proof is closed if it only contains closed literals. A positive literal $\psi$ is provable in $P$, notation $P \vdash \psi$, if there exists a proof of $\psi$ from $P$.

THEOREM 3.10. Let $P=(\Sigma, A, R)$ be a positive $T S S, \rightarrow_{P}$ the transition relation associated with $P$ and $\psi \in \operatorname{Tr}(\Sigma, A)$. Then

$$
P \vdash \psi \Leftrightarrow \psi \in \rightarrow_{P}
$$

PROOF

$\Rightarrow$ By straightforward induction on the proof of $\psi$ from $P$. 
$\Leftarrow$ It is straightforward to show that $\{\psi \mid P \vdash \psi\}$ is a model of $P$. As $\rightarrow_{P}$ is the least model of $P$, it follows that $\rightarrow_{P} \subseteq\{\psi \mid P \vdash \psi\}$.

From this theorem it also follows that the least model of a positive TSS is supported by it.

For TSSs with negative premises it is much more difficult to find an appropriate associated transition relation as is shown by the following example:

Example 3.11. Suppose we have a TSS $P$ with one constant $f$, two labels $a$ and $b$ and the following rules:

$$
\frac{f \stackrel{a}{\rightarrow} f}{f \stackrel{a}{\rightarrow} f} \quad \frac{f \stackrel{a}{\rightarrow}}{f \stackrel{b}{\rightarrow} f} .
$$

We would like $P$ to define the transition relation $\rightarrow_{P}=\{f \stackrel{b}{\rightarrow} f\}$. However, $P$ has exactly two minimal models, $\{f \stackrel{a}{\rightarrow} f\}$ and $\{f \stackrel{b}{\rightarrow} f\}$, which are both supported.

Thus, in the presence of negative premises, there may be several minimal models, some of which may be supported. So other characterizations for associated transition relations must be sought. The notion of provability also needs a revision, as it is not a priori clear how the negative premises of a rule must be proved.

Similar problems concerning negative premises have been studied in the context of logic programming. The correspondence between TSSs and logic programs is treated in Appendix A. A first solution proposed there introduced the notion of (local) stratification. Here we follow Groote [1993], where this notion was tailored for TSSs.

A TSS $P$ is stratified if there exists a stratification of the transitions with respect to the rules of $P$. The stratification guarantees that the validity of no literal depends on the validity of its negation.

Definition 3.12. Let $P=(\Sigma, A, R)$ be a TSS. A function $S: \operatorname{Tr}(\Sigma, A) \rightarrow \alpha$, where $\alpha$ is an ordinal, is called a stratification of $P$ if for every rule $r \in R$ and every substitution $\sigma: V \rightarrow T(\Sigma)$ it holds that:

$$
\text { for all } \psi \in \operatorname{pprem}(\sigma(r)): S(\psi) \leq S(\operatorname{conc}(\sigma(r)) \text { and }
$$

for all $t \stackrel{a}{\rightarrow} \in \operatorname{nprem}(\sigma(r))$ and $t^{\prime} \in T(\Sigma): S\left(t \stackrel{a}{\rightarrow} t^{\prime}\right)<S(\operatorname{conc}(\sigma(r)))$.

If $P$ has a stratification, we say that $P$ is stratified. For all ordinals $\beta<\alpha, S_{\beta}=$ $\{\varphi \mid S(\varphi)=\beta\}$ is called a stratum.

Example 3.13. The TSS of Example 3.11 can be stratified by a stratification $S$ as follows:

$$
S(f \stackrel{a}{\rightarrow} f)=0 \text { and } S(f \stackrel{b}{\rightarrow} f)=1 .
$$

Each positive transition system specification is trivially stratified by putting all positive literals in stratum 0. In Groote [1993] it is shown that $\mathrm{BPA}_{\epsilon \delta}$ with priorities and renaming but without abstraction is stratified under some appropriate conditions. 
We now define how a transition relation $\rightarrow_{P, S}$ is constructed from a TSS $P$ with stratification $S$, rephrasing a corresponding definition in Groote [1993]. The idea of the construction is that one first considers the positive literals in stratum 0 . As each literal in stratum 0 can only fit the conclusion of a rule without negative premises, one can determine which of these literals hold and which do not hold in $\rightarrow_{P, S}$ in the same way as is done for positive transition system specifications. If a literal in stratum 1 fits the conclusion of a rule, then this instance of that rule can only have negative premises in stratum 0 . If these negative premises hold (which has already been determined), they can be discarded. If they do not hold, the rule cannot be applied. Then we can prove the literals in stratum 1 in the ordinary way and we proceed with stratum 2, etc.

Definition 3.14. Let $P=(\Sigma, A, R)$ be a TSS with a stratification $S$ : $\operatorname{Tr}(\Sigma, A) \rightarrow \alpha$ for some ordinal $\alpha$. The transition relation $\rightarrow_{P, S}$ associated with $P$ (and based on $S$ ) is defined as:

$$
\rightarrow_{p, S}=\underset{0 \leq i<\alpha}{\cup} \rightarrow_{P_{i}}
$$

where $\rightarrow_{P_{i}}$ is defined by the (positive) TSS $P_{i}=\left(\Sigma, A, R_{i}\right)$ with $R_{i}$ given by:

$$
\begin{aligned}
& R_{i}=\left\{r^{\prime} \mid \exists r \in R \text { and } \exists \sigma: V \rightarrow T(\Sigma):\right. \\
& \bigcup_{0 \leq j<i} \rightarrow_{P_{j}} \vDash \operatorname{nprem}(\sigma(r)) \cup\{\varphi \in \operatorname{pprem}(\sigma(r)) \mid S(\varphi)<i\}, \\
& S(\operatorname{conc}(\sigma(r)))=i \text { and } \\
&\left.r^{\prime}=\frac{\{\varphi \in \operatorname{pprem}(\sigma(r)) \mid S(\varphi)=i\}}{\operatorname{conc}(\sigma(r))}\right\} .
\end{aligned}
$$

Theorem 3.15 (See Lemma 2.5.4 In Groote [1993]). Let $P$ be a TSS that is stratified by stratifications $S$ and $S^{\prime}$. Then $\rightarrow_{P, S}=\rightarrow_{P, S^{\prime}}$.

This theorem allows us to write $\rightarrow_{P}$ for the transition relation associated with a stratified TSS $P$. Note that the definition of $\rightarrow_{P}$ based on the notion of 'stratification' extends the definition of $\rightarrow_{P}$ for positive TSSs.

Theorem 3.16 (See Theorem 2.5.3 in Groote [1993] And Theorem 4 In PRZYMUSINSKI [1987]). Let $P$ be a stratified TSS. Then $\rightarrow_{P}$ is a minimal and supported model of $P$.

Thus, we have the scheme of characterizations depicted in Figure 1, where $A$ $\rightarrow B$ means that characterization $A$ implies characterization $B$. For positive TSSs, the characterizations marked by a $*$ coincide.

Although the stratification technique is often applicable, there are examples of TSSs that have an intuitive meaning while not being stratified. One such example is $\mathrm{BPA}_{\delta \epsilon \tau}$ with priorities.

Example 3.17. Suppose we have an instance $P_{\theta}$ of $\mathrm{BPA}_{\delta \epsilon \tau}$ with priorities based on a set of actions $A c t$ containing at least two elements $a$ and $b$ such that $a<b$. Consider for arbitrary terms $t$ and $u$ the following instances of rules: 


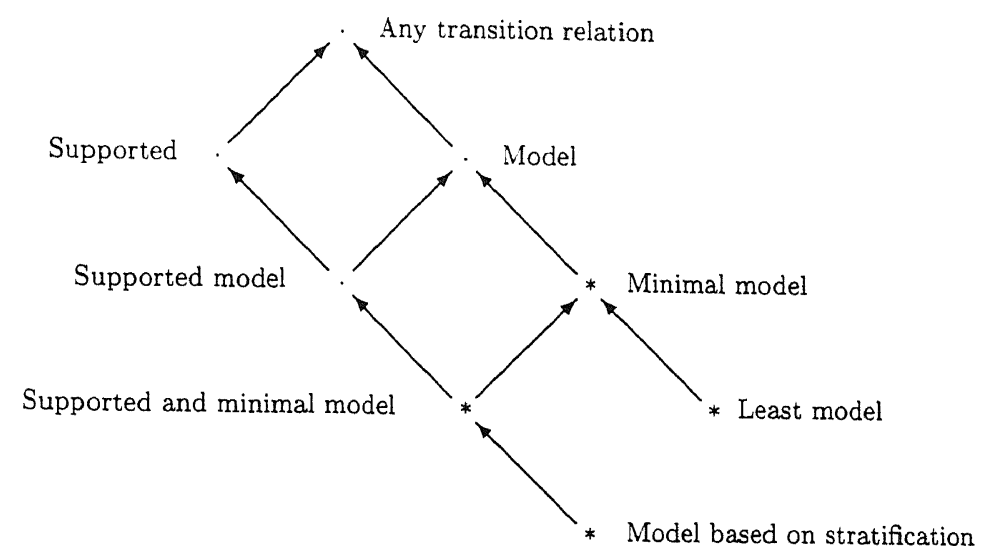

FIG. 1. Relations among several models.

$$
\begin{aligned}
& \text { R5.1: } \quad \frac{t \stackrel{a}{\rightarrow} u t \stackrel{b}{\rightarrow}}{\theta(t) \stackrel{a}{\rightarrow} \theta(u)}, \\
& \text { R7.2: } \frac{\theta(t) \stackrel{a}{\rightarrow} \theta(u)}{\tau_{\{a\}}(\theta(t)) \stackrel{\tau}{\rightarrow} \tau_{\{a\}}(\theta(u))}, \\
& \text { R9.3: } \quad \frac{t \stackrel{b}{\rightarrow} \tau_{\{a\}}(\theta(t)) \tau_{\{a\}}(\theta(t)) \stackrel{\tau}{\rightarrow} \tau_{\{a\}}(\theta(u))}{t \stackrel{b}{\rightarrow} \tau_{\{a\}}(\theta(u))} .
\end{aligned}
$$

For any stratification $S$ of $P_{\theta}$ it should thus hold that

$$
\begin{aligned}
& S\left(t \stackrel{b}{\rightarrow} \tau_{\{a\}}(\theta(u))\right)<\quad(\mathrm{R} 5.1) \\
& S(\theta(t) \stackrel{a}{\rightarrow} \theta(u)) \leq \\
& S\left(\tau_{\{a\}}(\theta(t)) \stackrel{\tau}{\rightarrow} \tau_{\{a\}}(\theta(u))\right) \leq(\mathrm{R} 7.2) \\
& S\left(t \stackrel{b}{\rightarrow} \tau_{\{a\}}(\theta(u))\right) .
\end{aligned}
$$

Of course, such a stratification cannot exist.

Again, this problem has been recognized earlier in logic programming, and several more powerful techniques were introduced there (see Apt and Bol [1994] for an overview). In the following two sections we adapt Gelfond and Lifschitz [1988] and van Gelder et al. [1988] for TSSs.

\section{TSSs and their associated transition relations}

So far no meaning has been given to TSSs that are not stratified. There are, however, TSSs, like BPA ${ }_{\delta \epsilon \tau}$ with priorities, that seem to be perfectly meaningful while not being stratified. This brings us back to the fundamental question of what transition relation should be associated with a TSS. Our answer is essentially that the transition relation must be the unique stable model in the sense of logic programming [Gelfond and Lifschitz 1988]. We strongly believe that any 
TSS that has no unique stable transition relation does not have a proper meaning.

The definition of a stable transition relation is intuitively as follows. Our first observation is that positive and negative premises in a rule of a TSS $P$ have a different status. In order to prove the conclusion of a rule, the positive premises of the rule must be proved from $P$. However, as $P$ contains only rules defining which literals hold, but not which literals do not hold, negative premises must be treated differently.

Conceptually, $t^{a} \rightarrow$ holds by default, that is, if for no $t^{\prime}: t \stackrel{a}{\rightarrow} t^{\prime}$ can be proved. But we are still trying to determine which literals can be proved. So instead of an immediate characterization of the set of provable literals $\rightarrow$, we have an equation with this set both on the left and on the right side, namely,

$\rightarrow$ equals the set of literals that are provable by those rules of the TSS of which the negative premises hold in $\rightarrow$.

This equation does not give us a means to compute the transition relation $\rightarrow$, but we can easily check whether a given transition relation satisfies our criterion.

We now formalize these ideas. In Sections 4,5 , and 6 we use only ground TSSs, that is, we identify a set of rules $R$ with the set of ground instances of $R$.

Definition 4.1. Let $P=(\Sigma, A, R)$ be a TSS. Let $\rightarrow \subseteq \operatorname{Tr}(\Sigma, A)$.

$$
\operatorname{Strip}(P, \rightarrow)=(\Sigma, A, \operatorname{Strip}(R, \rightarrow))
$$

where

$$
\operatorname{Strip}(R, \rightarrow)=\left\{r^{\prime} \mid \exists r \in R: \rightarrow \vDash n \operatorname{npem}(r) \text { and } r^{\prime}=\frac{\operatorname{pprem}(r)}{\operatorname{conc}(r)}\right\}
$$

Given a transition relation $\rightarrow$, the function Strip removes all rules in $R$ that have negative premises that do not hold in $\rightarrow$. Furthermore, it drops the negative premises from the remaining rules. The following lemma is therefore obvious.

LEMmA 4.2. Let $P=(\Sigma, A, R)$ be a TSS and let $\rightarrow \subseteq \operatorname{Tr}(\Sigma, A)$ be a transition relation. Then $\operatorname{Strip}(P, \rightarrow)$ is a positive TSS.

Using the fact that the notion of provability is already captured in the definition of the transition relation associated with a positive TSS, we can now easily formalize the previously stated equation.

Definition 4.3 (Stable transition relation). Let $P=(\Sigma, A, R)$ be a TSS. A transition relation $\rightarrow \subseteq \operatorname{Tr}(\Sigma, A)$ is stable for $P$ if $\rightarrow=\rightarrow_{\operatorname{Strip}(P, \rightarrow)}$.

Remark 4.4. In general, for a TSS $P$ there may be 0,1 or more transition relations that are stable for $P$, for example: 


$$
\begin{aligned}
& 0: \frac{f \stackrel{a}{\rightarrow}}{f \stackrel{a}{\rightarrow} f} \\
& \text { 1: } \frac{f \stackrel{a}{\rightarrow} f \stackrel{b}{\rightarrow} f}{f \stackrel{a}{\rightarrow} f} \quad[\rightarrow=\emptyset] \\
& \text { 2: } \frac{f \stackrel{a}{\rightarrow}}{f \stackrel{b}{\rightarrow} f} \frac{f \stackrel{b}{\rightarrow}}{f \stackrel{a}{\rightarrow} f} \quad[\rightarrow=\{f \stackrel{a}{\rightarrow} f\} \text { or } \rightarrow=\{f \stackrel{b}{\rightarrow} f\}]
\end{aligned}
$$

We do not have any idea as to which transition relations should be associated with the first TSS, nor do we know which one of the two transition relations of the third TSS should be preferred. In fact we think that there are no satisfying answers to those questions. Thus, we propound the following definition:

Definition 4.5. Let $P$ be a TSS. If there is a unique transition relation $\rightarrow$ stable for $P$, then $\rightarrow$ is the transition relation associated with $P$.

In order to avoid confusion, we do not again introduce the notation $\rightarrow_{P}$ : until Section 7 this notation remains reserved for stratified TSSs.

Remark 4.6. If $P$ is positive, then for every transition relation $\rightarrow, \operatorname{Strip}(P$, $\rightarrow)=P$, thus $\rightarrow_{P}$ is the unique transition relation that is stable for $P$. Hence, this definition of 'associated with' coincides with the previously given definition for positive TSSs. In Section 6, we show that our choice also extends the definition of 'associated with' for stratified TSSs.

The following lemma will be used implicitly in almost every proof to follow. Moreover, it shows that our choice that a transition relation must be stable for a TSS is also a refinement of the requirement that a transition relation must be a supported and minimal model of it.

Lemma 4.7. Let $P=(\Sigma, A, R)$ be a TSS and let $\rightarrow \subseteq \operatorname{Tr}(\Sigma, A)$ be a transition relation. If $\rightarrow$ is stable for $P$, then

(1) $\rightarrow$ is a model of $P$,

(2) $\rightarrow$ is supported by $P$,

(3) $\rightarrow$ is a minimal model of $P$ (cf. [Gelfond and Lifschitz 1988; Theorem 1]).

Proof. Let $\rightarrow$ be a transition relation that is stable for $P$.

(1) Suppose $r \in R$ and $\rightarrow \vDash \operatorname{prem}(r)$. Hence

$$
\frac{\operatorname{pprem}(r)}{\operatorname{conc}(r)} \in \operatorname{Strip}(R, \rightarrow) \text {. }
$$

As $\rightarrow=\rightarrow_{\operatorname{strip}(P, \rightarrow)}$ is a model of $\operatorname{Strip}(P, \rightarrow)$ and $\rightarrow \vDash \operatorname{pprem}(r), \rightarrow \vDash$ $\operatorname{conc}(r)$.

(2) Suppose $\varphi \in \rightarrow$. Hence, $\rightarrow \vDash \varphi$ and thus $\rightarrow_{\operatorname{Strip}(P, \rightarrow)} \vDash \varphi$. This means that there is a proof for $\varphi$ using the rules in $\operatorname{Strip}(R, \rightarrow)$. Assume rule $r$ is the last rule used. So $\operatorname{conc}(r)=\varphi$. Hence, $\operatorname{Strip}(P, \rightarrow) \vdash \operatorname{prem}(r)$ and thus $\rightarrow$ $\vDash \operatorname{prem}(r)$. As $r \in \operatorname{Strip}(R, \rightarrow)$ there is a rule 


$$
r^{\prime}=\frac{\operatorname{prem}(r) \cup \text { nprem }}{\operatorname{conc}(r)} \in R
$$

where nprem is a set of negative premises such that $\rightarrow \vDash n$ prem. Hence, for this rule $r^{\prime} \in R$ it holds that $\varphi=\operatorname{conc}\left(r^{\prime}\right)$ and $\rightarrow \vDash \operatorname{prem}\left(r^{\prime}\right)$. Hence, $\rightarrow$ is supported by $P$.

(3) We must show that $\rightarrow$ is minimal among the models of $P$. Suppose $\rightarrow^{*} \subseteq \rightarrow$ is also a model of $P$. We show that $\rightarrow^{*}$ is a model of $\operatorname{Strip}(P, \rightarrow)$. Let $r$ be a rule of $\operatorname{Strip}(P, \rightarrow)$. This means that there is some

$$
r^{\prime}=\frac{\operatorname{prem}(r) \cup \text { nprem }}{\operatorname{conc}(r)} \in R
$$

for some set nprem of negative premises. As $\rightarrow^{*} \subseteq \rightarrow$ and $\rightarrow \vDash n$ prem,$\rightarrow^{*}$ $\vDash$ nprem. As $\rightarrow^{*}$ is a model of $P$, we have

$$
\text { if } \rightarrow^{*} \vDash \operatorname{prem}(r) \cup \text { nprem, then } \rightarrow^{*} \vDash \operatorname{conc}(r) \text {. }
$$

Knowing that $\rightarrow^{*} \vDash$ nprem, this reduces to

$$
\text { if } \rightarrow^{*} \vDash \operatorname{prem}(r) \text {, then } \rightarrow^{*} \vDash \operatorname{conc}(r) \text {. }
$$

Thus $\rightarrow^{*}$ is a model for every rule $r$ in $\operatorname{Strip}(P, \rightarrow)$. As $\rightarrow$ is the least model of $\operatorname{Strip}(P, \rightarrow)$, it follows that $\rightarrow^{*}=\rightarrow$.

We show how the notion is stable for can be applied to our running example. What we in fact show is that in general there is no stable transition relation for $\mathrm{BPA}_{\delta \epsilon \tau}$ with priorities instantiated with a set of process declarations where the abstraction operator $\tau_{I}$ is allowed in process terms.

Example 4.8. Consider $P_{\theta}$ with at least two actions $a$ and $b$ such that $a>b$ and a process name $X$ with the recursive definition

$$
X \Leftarrow \theta\left(\tau_{\{b\}}(X) \cdot a+b\right) \in E .
$$

Now assume that there is a relation $\rightarrow$ that is stable for $P_{\theta}$. We show that this assumption leads to a contradiction. For a more convenient notation, we use $t \stackrel{a}{\rightarrow}$ as an abbreviation of $\exists u \in T\left(\Sigma_{\theta}\right): \rightarrow \vDash t \stackrel{a}{\rightarrow} u\left(t \in T\left(\Sigma_{\theta}\right), a \in A_{\theta}\right)$. We distinguish three cases but we do not present them in full detail. In particular, not all possible applications of R9.2 and R9.3 are considered explicitly.

$-\tau_{\{b\}}(X) \stackrel{\vee}{\rightarrow}$. As $\rightarrow$ is a model of $P_{\theta}$, we have that $\tau_{\{b\}}(X) \cdot a \stackrel{a}{\rightarrow}$ (by rule $\mathrm{R} 4.2$ ) and hence $\tau_{\{b\}}(X) \cdot a+b \stackrel{a}{\rightarrow}$ (by rule R3.1). Thus $\theta\left(\tau_{\{b\}}(X) \cdot a+b\right) \stackrel{a}{\rightarrow}$ and $\rightarrow \vDash \theta\left(\tau_{\{b\}}(X) \cdot a+b\right) \stackrel{b}{\rightarrow}$ (by rule R5.1). So $\rightarrow \vDash X \stackrel{b}{\rightarrow}$. As obviously $\rightarrow \vDash X \stackrel{\vee}{\rightarrow}$ ( $X$ must perform at least an $a$ or $b$ action), it follows that $\rightarrow \vDash$ $\tau\{b\}(X) \forall$. Contradiction.

$-\rightarrow \vDash \tau_{\{b\}}(X) \stackrel{\vee}{\rightarrow}$ and $\rightarrow \vDash \tau_{\{b\}}(X) \stackrel{a}{\ngtr} \rightarrow$. Then obviously $\rightarrow \vDash \tau_{\{b\}}(X) \cdot a+b \stackrel{a}{\Rightarrow}$, so $\rightarrow \models \theta\left(\tau_{\{b\}}(X) \cdot a+b\right) \stackrel{b}{\rightarrow} \theta(\epsilon) \quad$ (using R2, R3.2 and R5.1). Hence, $\rightarrow \vDash X \stackrel{b}{\rightarrow} \theta(\epsilon)$, so $\rightarrow \vDash \tau_{\{b\}}(X) \stackrel{\vee}{\rightarrow} \tau_{\{b\}}(\theta(\delta)$ ) (using R8, R7.2, R1, R5.2, R7.2 and R9.2). Contradiction. 
$-\rightarrow \vDash \tau_{\{b\}}(X) \stackrel{\vee}{\rightarrow \rightarrow}$ and $\tau_{\{b\}}(X) \stackrel{a}{\rightarrow} t$ for some $t \in T\left(\Sigma_{\theta}\right)$. This can also not be the case as there is no proof for $\operatorname{Strip}\left(P_{\theta}, \rightarrow\right) \vdash \tau_{\{b\}}(X) \stackrel{a}{\rightarrow} t$. In order to prove $\tau_{\{b\}}(X) \stackrel{a}{\rightarrow} t$, we must show that $X \stackrel{a}{\rightarrow}$, and in order to show this we need $\tau_{\{b\}}(X) \cdot a \stackrel{a}{\rightarrow}$. In combination with the assumption that $\tau_{\{b\}}(X) \nsucc$, this requires $\tau_{\{b\}}(X) \stackrel{a}{\rightarrow}$ again. Thus the most 'reasonable' attempt to construct the required proof loops. All other attempts to prove $\tau_{\{b\}}(X) \stackrel{a}{\rightarrow} t$, for example, via R9.3:

$$
\frac{\tau_{\{b\}}(X) \stackrel{a}{\rightarrow} u \stackrel{\sim}{\rightarrow} t}{\tau_{\{b\}}(X) \stackrel{a}{\rightarrow} t},
$$

also loop.

\section{Reducing TSSs}

We now present a technique that can be useful for proving that a certain TSS has a unique stable transition relation. This technique is inspired by the well-founded models that are introduced in van Gelder et al. [1988]. First we construct a 3-valued 'interpretation' for a TSS $P$, partitioning the set of transitions in three groups: those that are certainly true, those of which the truth is unknown and those that are certainly not true. We apply this information to reduce $P$ to another TSS with exactly the same stable transition relations as $P$. In this new TSS, the truth or falsity of more literals may become certain. Repeated reduction may lead to complete information: the unique stable transition relation.

If in the next definition $\rightarrow_{\text {true }}$ contains transitions that certainly hold and $\rightarrow_{\text {pos }}$ contains all transitions that possibly hold, then rules with certainly wrong premises are removed and in the remaining rules all premises that certainly hold are dropped.

Definition 5.1. Let $P=(\Sigma, A, R)$ be a TSS. Let $\rightarrow_{\text {true }}, \rightarrow_{p o s} \subseteq \operatorname{Tr}(\Sigma, A)$ be transition relations.

$$
\operatorname{Reduce}\left(P, \rightarrow_{\text {true }}, \rightarrow_{\text {pos }}\right)=\left(\Sigma, A, \operatorname{Reduce}\left(R, \rightarrow_{\text {true }}, \rightarrow_{\text {pos }}\right)\right),
$$

where

$$
\begin{gathered}
\operatorname{Reduce}\left(R, \rightarrow_{\text {true }}, \rightarrow_{\text {pos }}\right)=\left\{r^{\prime} \mid \exists r \in R: \rightarrow_{\text {true }} \vDash \operatorname{nprem}(r), \rightarrow_{\text {pos }} \vDash \operatorname{pprem}(r)\right. \text { and } \\
\left.r^{\prime}=\frac{\left\{\psi \in \operatorname{pprem}(r)\left|\rightarrow_{\text {true }}\right| \neq \psi\right\} \cup\left\{\psi \in \operatorname{nprem}(r) \mid \rightarrow_{\text {pos }} \not \psi \psi\right\}}{\operatorname{conc}(r)}\right\} .
\end{gathered}
$$

Thus, the reduction of a rule consists of two phases. First, it is checked that the premises are possibly true. For positive premises, this is straightforward: $t \stackrel{a}{\rightarrow} t^{\prime}$ is possibly true if $t \stackrel{a}{\rightarrow} t^{\prime} \in \rightarrow_{p o s}$. Hence, the condition $\rightarrow_{p o s} \vDash \operatorname{pprem}(r)$. A negative premise $t \stackrel{a}{t}$ is possibly true if it is not certain that $t$ can perform an $a$-step, that is, for no $t^{\prime}$ it is certain that $t \stackrel{a}{\rightarrow} t^{\prime}$ holds. Thus, $t \stackrel{a}{\rightarrow}$ is possibly true if for no $t^{\prime}, t \stackrel{a}{\rightarrow} t^{\prime} \in \rightarrow_{\text {true }}$. Hence the condition $\rightarrow_{\text {true }} \vDash n$ prem $(r)$.

If indeed the premises of the rule are possibly true, then the premises that are certainly true are removed. A positive premise $t \stackrel{a}{\rightarrow} t^{\prime}$ is certainly true if $t \stackrel{a}{\rightarrow} t^{\prime} \in$ $\rightarrow_{\text {true }}$. A negative premise $t \stackrel{a}{\rightarrow}$ is certainly true if $t$ cannot possibly perform an 
$a$-step, that is, for no $t^{\prime}: t \stackrel{a}{\rightarrow} t^{\prime}$ is possible. Thus $t \stackrel{a}{\rightarrow}$ is certainly true if $\rightarrow_{\text {pos }} \vDash$ $t^{a} \rightarrow$. We shall always use Definition 5.1 with $\rightarrow_{\text {true }} \subseteq \rightarrow_{\text {pos }}$ (cf. Lemma 5.5).

Remark 5.2. Note that $\operatorname{Reduce}(R, \rightarrow, \rightarrow)$ differs from $\operatorname{Strip}(R, \rightarrow)$. In $\operatorname{Strip}(R, \rightarrow)$ only negative premises are checked, yielding a positive TSS; in $\operatorname{Reduce}(R, \rightarrow, \rightarrow)$ all premises are checked, resulting in a TSS consisting solely of rules without premises.

The 3-valued interpretation required is obtained by means of two positive TSSs: True $(P)$ and $\operatorname{Pos}(P)$. True $(P)$ determines the transitions that are certainly true: the transitions that can be proved with positive rules only. $\operatorname{Pos}(P)$ determines the transitions that are possibly true, that is, true or unknown. These are the transitions that can be proved ignoring negative premises. Thus, $\operatorname{Pos}(P)$ is obtained from $P$ by removing all negative premises of the rules.

Definition 5.3. Let $P=(\Sigma, A, R)$ be a TSS.

$-\operatorname{True}(P)=(\Sigma, A, \operatorname{True}(R))$ where $\operatorname{True}(R)=\{r \in R \mid n \operatorname{prem}(r)=\emptyset\}$.

$-\operatorname{Pos}(P)=(\Sigma, A, \operatorname{Pos}(R))$ where

$$
\operatorname{Pos}(R)=r^{\prime} \mid \exists r \in R: r^{\prime}=\frac{\operatorname{pprem}(r)}{\operatorname{conc}(r)} .
$$

Because after the reduction of $P$ the truth or falsity of more literals may become certain, it is worthwhile to iterate the reduction process; if necessary even transfinitely many reduction steps may be considered.

Definition 5.4. Let $P=(\Sigma, A, R)$ be a TSS. For every ordinal $\alpha$, the $\alpha$-reduction of $P$, notation $\operatorname{Red}^{\alpha}(P)$, is recursively defined as follows:

- $\operatorname{Red}^{0}(P)=\left(\Sigma, A, R_{\text {ground }}\right)$ where $R_{\text {ground }}$ is the set of all ground instances of rules in $R$,

$-\operatorname{Red}^{\alpha}(P)=\operatorname{Reduce}\left(P, \cup_{\beta<\alpha} \rightarrow_{\operatorname{True}\left(\operatorname{Red}^{\beta}(P)\right)}, \cap_{\beta<\alpha} \rightarrow_{\operatorname{Pos}\left(\operatorname{Red}^{\beta}(P)\right)}\right)$.

Thus, in contrast with van Gelder et al. [1988] and general practice in logic programming, our operator maps TSSs to TSSs rather than interpretations to interpretations; for details see Appendix A. This allows us in Section 6 to combine reduction with stratification: as soon as the reduced TSS is stratified, no further reduction is needed.

The following lemma plays an important role in a number of proofs to follow. It shows that the reduction process can never make a certainly true (or false) literal become unknown. Thus reduction is monotonic in this sense.

Lemma 5.5 (Monotonicity of Reduction). Let $P=(\Sigma, A, R)$ be a TSS. For all ordinals $\beta$ and $\alpha$ such that $\beta<\alpha$ and for every $\rightarrow \subseteq \operatorname{Tr}(\Sigma, A)$ :

$$
\begin{aligned}
\rightarrow_{\text {rue }\left(\operatorname{Red} d^{\beta}(P)\right)} \subseteq & \rightarrow_{\operatorname{True}\left(\operatorname{Red}^{\alpha}(P)\right)} \subseteq \\
& \rightarrow_{\operatorname{Strip}\left(\operatorname{Red}^{\alpha}(P), \rightarrow\right)} \subseteq \\
& \rightarrow_{\operatorname{Pos}\left(\operatorname{Red}^{\alpha}(P)\right)} \subseteq \rightarrow_{\operatorname{Pos}\left(\operatorname{Red}^{\beta}(P)\right)}
\end{aligned}
$$




\section{PROOF}

(1) First we show that $\rightarrow_{\text {True }\left(\operatorname{Red}^{\alpha}(P)\right)} \subseteq \rightarrow_{\operatorname{Strip}\left(\operatorname{Red}^{\alpha}(P), \rightarrow\right)} \subseteq \rightarrow_{P o s\left(\operatorname{Red}^{\alpha}(P)\right)}$. For every TSS $P^{\prime}=\left(\Sigma, A, R^{\prime}\right): \operatorname{True}\left(R^{\prime}\right) \subseteq \operatorname{Strip}\left(R^{\prime}, \rightarrow\right) \subseteq \operatorname{Pos}\left(R^{\prime}\right)$. As these TSSs are all positive, $\rightarrow_{\text {True }\left(P^{\prime}\right)} \subseteq \rightarrow_{\operatorname{Strip}\left(P^{\prime}, \rightarrow\right)} \subseteq \rightarrow_{\operatorname{Pos}\left(P^{\prime}\right)}$. Now taking $P^{\prime}=\operatorname{Red}^{\alpha}(P)$ proves this case.

(2) Here it is shown that $\rightarrow_{\operatorname{Pos}\left(\operatorname{Red}^{\alpha}(P)\right)} \subseteq \rightarrow_{\operatorname{Pos}\left(\operatorname{Red}^{\beta}(P)\right)}$. Suppose $\operatorname{Pos}\left(\operatorname{Red}^{\alpha}(P)\right)$ $\vdash \varphi$. Then there is a rule $r^{\prime} \in \operatorname{Pos}\left(\operatorname{Red}^{\alpha}(P)\right)$ such that $\operatorname{conc}\left(r^{\prime}\right)=\varphi$ and $\operatorname{Pos}\left(\operatorname{Red}^{\alpha}(P)\right) \vdash \operatorname{prem}\left(r^{\prime}\right)$. Hence, there is a rule $r \in R$ such that $\operatorname{conc}(r)$ $=\varphi$ and

$$
\begin{gathered}
\bigcup_{\gamma<\alpha} \rightarrow_{\operatorname{True}(\operatorname{Red} \gamma(P))} \vDash \operatorname{nprem}(\boldsymbol{r}), \\
\bigcap_{\gamma<\alpha} \rightarrow_{\operatorname{Pos}\left(\operatorname{Red}^{\gamma}(P)\right)} \vDash \operatorname{pprem}(r) .
\end{gathered}
$$

Now

$$
\begin{aligned}
& \bigcup_{\gamma<\beta} \rightarrow_{\operatorname{True}(\operatorname{Red} \gamma(P))} \subseteq \bigcup_{\gamma<\alpha} \rightarrow_{\operatorname{True}(\operatorname{Red} \gamma(P))}, \\
& \bigcap_{\gamma<\beta} \rightarrow_{\operatorname{Pos}\left(\operatorname{Red}^{\gamma}(P)\right)} \supseteq \bigcap_{\gamma<\alpha} \rightarrow_{\operatorname{Pos}(\operatorname{Red} \gamma(P)) .}
\end{aligned}
$$

Hence, $\cup_{\gamma<\alpha} \rightarrow_{\text {True }\left(\operatorname{Red}^{\gamma}(P)\right)} \vDash \operatorname{nprem}(r)$ implies that $\cup_{\gamma<\beta} \rightarrow_{\operatorname{True}\left(\operatorname{Red}^{\gamma}(P)\right)}$ $\vDash$ nprem $(r)$. Conversely, $\bigcap_{\gamma<\alpha} \rightarrow_{\operatorname{Pos}\left(\operatorname{Red}^{\gamma}(P)\right)} \vDash \operatorname{pprem}(r)$ implies $\cap_{\gamma<\beta}$ $\rightarrow_{\operatorname{Pos}\left(\operatorname{Red}^{\gamma}(P)\right)} \vDash \operatorname{pprem}(r)$. Hence $\operatorname{Pos}\left(\operatorname{Red}^{\beta}(R)\right)$ contains a rule $r^{\prime \prime}$ such that $\operatorname{prem}\left(r^{\prime \prime}\right) \subseteq \operatorname{pprem}(r)$ and $\operatorname{conc}\left(r^{\prime \prime}\right)=\varphi$. As also $\cap_{\gamma<\alpha} \rightarrow_{\operatorname{Pos}\left(\operatorname{Red}^{\gamma}(P)\right)}$ $\vDash \operatorname{pprem}(r)$ implies $\rightarrow_{\operatorname{Pos}\left(\operatorname{Red} d^{\beta}(P)\right)} \vDash \operatorname{pprem}(r)$, it holds that $\rightarrow_{\operatorname{Pos}\left(\operatorname{Red} d^{\beta}(P)\right)}$ $\vDash \operatorname{prem}\left(r^{\prime \prime}\right)$. Thus, $\operatorname{Pos}\left(\operatorname{Red}^{\beta}(P)\right) \vdash \varphi$.

(3) We are left to show $\rightarrow_{\operatorname{True}\left(\operatorname{Red}^{\beta}(P)\right)} \subseteq \rightarrow_{\operatorname{True}\left(\operatorname{Red}^{\alpha}(P)\right)}$. We show this by induction on $\alpha$. By induction we may assume that:

$$
\text { for all } \zeta \leq \gamma<\alpha: \rightarrow_{\text {True }(\operatorname{Red}(P))} \subseteq \rightarrow_{\text {True }(\operatorname{Red} \gamma(P))} .
$$

Suppose $\operatorname{True}\left(\operatorname{Red}^{\beta}(P)\right) \vdash \varphi$. Then, there is an instance $r$ of a rule in $R$ such that $\operatorname{conc}(r)=\varphi$,

$$
\bigcap_{\gamma<\beta} \rightarrow_{\operatorname{Pos}(\operatorname{Red}(P))} \vDash \operatorname{nprem}(r)
$$

(all negative premises of $r$ must be removed by reduction) and

$$
\bigcup_{\gamma \leq \beta} \rightarrow_{\text {True }(\operatorname{Red} \gamma(P))} \vDash \operatorname{pprem}(r)
$$

(all positive premises of $r$ must be removed by reduction or proved in True $\left.\left(\operatorname{Red}^{\beta}(P)\right)\right)$. From this, using the induction hypothesis (i.h.) and (1) and (2) above, we can infer the following two facts:

$$
\begin{aligned}
& -\cap_{\gamma<\beta} \rightarrow_{P o s\left(\operatorname{Red}^{\gamma}(P)\right)} \vDash \operatorname{nprem}(r) \Rightarrow \\
& \cap_{\gamma \leq \beta} \rightarrow_{P o s\left(\operatorname{Red}^{\gamma}(P)\right)} \vDash \operatorname{nprem}(r) \stackrel{(2)}{\Rightarrow} \\
& \rightarrow_{P o s\left(\operatorname{Red}^{\beta}(P)\right)} \vDash \operatorname{nprem}(r) \stackrel{(2)}{\Rightarrow} \\
& \forall \gamma(\beta \leq \gamma<\alpha): \rightarrow_{\operatorname{Pos}\left(\operatorname{Red}^{\gamma}(P)\right)} \vDash \operatorname{nprem}(r) \stackrel{(1)}{\Rightarrow} \\
& \forall \gamma(\beta \leq \gamma<\alpha): \rightarrow_{\text {True }\left(\operatorname{Red}^{\gamma}(P)\right)} \vDash \operatorname{nprem}(r) \stackrel{(i . h .)}{\Rightarrow} \\
& \forall \gamma<\alpha: \rightarrow_{\text {True }\left(\operatorname{Red}^{\gamma}(P)\right)} \vDash \operatorname{nprem}(r) \Rightarrow
\end{aligned}
$$




$$
\begin{aligned}
& \cup_{\gamma<\alpha} \rightarrow_{\text {True }\left(\operatorname{Red}^{\gamma}(P)\right)} \vDash \operatorname{nprem}(r) . \\
& -U_{\leq \beta} \rightarrow_{\text {True }\left(\operatorname{Red}^{\gamma}(P)\right)} \vDash \operatorname{pprem}(r) \stackrel{(i . h .)}{\Rightarrow} \\
& \rightarrow_{\text {True }\left(\operatorname{Red}^{\beta}(P)\right)} \vDash \operatorname{pprem}(r) \stackrel{(i . h)}{\Rightarrow} \\
& \forall \gamma(\beta \leq \gamma<\alpha): \rightarrow_{\text {True }\left(\operatorname{Red}^{\gamma}(P)\right)} \vDash \operatorname{pprem}(r) \stackrel{(1)}{\Rightarrow} \\
& \forall \gamma(\beta \leq \gamma<\alpha): \rightarrow_{P o s\left(\operatorname{Red}^{\gamma}(P)\right)} \vDash \operatorname{pprem}(r) \stackrel{(2)}{\Rightarrow} \\
& \forall \gamma<\alpha: \rightarrow_{P o s\left(\operatorname{Red}^{\gamma}(P)\right)} \vDash \operatorname{pprem}(r) \Rightarrow \\
& \cap \gamma<\alpha \rightarrow_{\operatorname{Pos}\left(\operatorname{Red}^{\gamma}(P)\right)} \vDash \operatorname{pprem}(r) \text {. }
\end{aligned}
$$

$\langle 1\rangle$ and $\langle 2\rangle$ imply

$$
\exists r^{\prime}=\frac{\operatorname{prem}(r)-\cdots}{\varphi} \in \operatorname{Red}^{\alpha}(R) .
$$

Furthermore,

$$
\begin{gathered}
\cap_{\gamma<\beta} \rightarrow_{\operatorname{Pos}_{\left(\operatorname{Red}^{\gamma}(P)\right)}} \vDash \operatorname{nprem}(r) \Rightarrow \\
\cap_{\gamma<\alpha} \rightarrow_{\operatorname{Pos}_{\left(\operatorname{Red}^{\gamma}(P)\right)}} \vDash \operatorname{nprem}(r) \Rightarrow \\
n \operatorname{nprem}\left(r^{\prime}\right)=\emptyset \Rightarrow \\
r^{\prime} \in \operatorname{True}\left(\operatorname{Red}^{\alpha}(R)\right) .
\end{gathered}
$$

As for every $\psi \in \operatorname{prem}\left(r^{\prime}\right)$, the proof of $\psi$ in $\operatorname{True}\left(\operatorname{Red}^{\beta}(P)\right)$ is less deep than the proof of $\varphi$ in $\operatorname{True}\left(\operatorname{Red}^{\beta}(P)\right)$, we may conclude by induction that $\operatorname{prem}\left(r^{\prime}\right) \subseteq \rightarrow_{\text {True }\left(\operatorname{Red}^{\alpha}(P)\right)}$. Hence, $\operatorname{conc}\left(r^{\prime}\right)=\varphi \in \rightarrow_{\text {True }}\left(\operatorname{Red}^{\alpha}(P)\right)$.

In order to apply this reduction process, we also need the following lemma.

Lemma 5.6. Let $P=(\Sigma, A, R)$ be a TSS and let $\rightarrow \subseteq \operatorname{Tr}(\Sigma, A)$. For all ordinals $\alpha$ implies

$$
\bigcup_{\beta<\alpha} \rightarrow_{\text {True }(\operatorname{Red}(P))} \subseteq \rightarrow \subseteq \underset{\beta<\alpha}{\bigcap} \rightarrow_{\operatorname{Pos}\left(\operatorname{Red}^{\beta}(P)\right)}
$$

$$
\rightarrow_{\operatorname{Strip}(P, \rightarrow)}=\rightarrow_{\operatorname{Strip}\left(\operatorname{Red}^{\alpha}(P), \rightarrow\right)}
$$

PROOF

$\longrightarrow \operatorname{Strip}_{(P, \rightarrow)} \subseteq \rightarrow_{\operatorname{Strip}\left(\operatorname{Red}^{\alpha}(P), \rightarrow\right)}$. Let $\psi \in \rightarrow_{\operatorname{Strip}(P, \rightarrow)}$. Hence, $\operatorname{Strip}(P, \rightarrow) \vdash$ $\psi$. We use induction on this proof. There is a rule $r^{\prime}$ in $\operatorname{Strip}(P, \rightarrow)$ such that $\operatorname{conc}\left(r^{\prime}\right)=\psi$ and $\rightarrow_{\operatorname{Strip}(P, \rightarrow)} \vDash \operatorname{prem}\left(r^{\prime}\right)$. Hence, there is a rule $r \in R$ such that

$$
r^{\prime}=\frac{\operatorname{pprem}(r)}{\operatorname{conc}(r)}
$$

and $\rightarrow \vDash n$ prem $(r)$. By induction on $\alpha$ and Lemma 5.5 it follows that

$$
\forall \beta<\alpha: \rightarrow_{\operatorname{Strip}(P, \rightarrow)}=\rightarrow_{\operatorname{Strip}(\operatorname{Red} \beta(P), \rightarrow)} \subseteq \rightarrow_{\operatorname{Pos}(\operatorname{Red} \beta(P))}
$$

and hence, 


$$
\begin{aligned}
& \left.\begin{array}{l}
\rightarrow \vDash \operatorname{nprem}(r) \\
\cup_{\beta<\alpha} \rightarrow_{\operatorname{True}(\operatorname{Red} \beta(P))} \subseteq \rightarrow
\end{array}\right\} \Rightarrow \cup_{\beta<\alpha} \rightarrow_{\operatorname{True}(\operatorname{Red} \beta(P))} \vDash \operatorname{nprem}(r), \\
& \left.\begin{array}{l}
\rightarrow_{\operatorname{Strip}(P, \rightarrow)} \vDash \operatorname{pprem}(r) \\
\rightarrow_{\operatorname{Strip}(P, \rightarrow)} \subseteq \cap_{\beta<\alpha} \rightarrow_{\operatorname{Pos}(\operatorname{Red} \beta(P))}
\end{array}\right\} \Rightarrow \cap_{\beta<\alpha} \rightarrow_{P o s(\operatorname{Red} \beta(P))} \vDash \operatorname{pprem}(r) .
\end{aligned}
$$

So there is a rule

$r=$

$\frac{\left\{\psi^{\prime} \in \operatorname{pprem}(r) \mid \cap_{\beta<\alpha} \rightarrow_{\operatorname{True}(\operatorname{Red}(P))} \not \neq \psi^{\prime}\right\} \cup\left\{\psi^{\prime} \in \operatorname{nprem}(r) \mid \cap_{\beta<\alpha} \rightarrow_{\left.\operatorname{Pos}_{(\operatorname{Red}}(P)\right)} \not \neq \psi^{\prime}\right\}}{\operatorname{conc}(r)}$

$\in \operatorname{Red}^{\alpha}(P)$

Furthermore, $\rightarrow \vDash n$ prem $\left(r^{\prime \prime}\right) \subseteq \operatorname{nprem}(r)$. So

$$
r^{\prime \prime \prime}=\frac{\left\{\psi^{\prime} \in \operatorname{pprem}(r) \mid \cup_{\beta<\alpha} \rightarrow_{\operatorname{True}\left(\operatorname{Red}^{\beta}(P)\right)} \not \neq \psi^{\prime}\right\}}{\operatorname{conc}(r)} \in \operatorname{Strip}\left(\operatorname{Red}^{\alpha}(P), \rightarrow\right) .
$$

By induction on the depth of the proof of $\psi$ from $\operatorname{Strip}(P, \rightarrow)$ we may assume that $\operatorname{prem}\left(r^{\prime}\right) \subseteq \rightarrow_{\operatorname{Strip}\left(\operatorname{Red}^{\alpha}(P), \rightarrow\right)}$, so $\rightarrow_{\operatorname{Strip}\left(\operatorname{Red} d^{\alpha}(P), \rightarrow\right)} \vDash$ $\operatorname{prem}\left(r^{\prime}\right)=\operatorname{pprem}(r) \supseteq \operatorname{prem}\left(r^{\prime \prime \prime}\right)$. Hence, it follows that $\operatorname{Strip}\left(\operatorname{Red}^{\alpha}(P), \rightarrow\right) \vDash$ $\operatorname{conc}\left(r^{\prime \prime \prime}\right)=\psi$.

$\longrightarrow$ Strip $(P, \rightarrow) \supseteq \rightarrow_{\operatorname{Strip}\left(\operatorname{Red}^{\alpha}(P, \rightarrow)\right)}$. Let $\psi \in \rightarrow_{\operatorname{Strip}\left(\operatorname{Red}^{\alpha}(P), \rightarrow\right)}$. Hence, it must be that $\operatorname{Strip}\left(\operatorname{Red}^{\alpha}(P), \rightarrow\right) \vdash \psi$. So there is a rule $r^{\prime}$ in $\operatorname{Strip}\left(\operatorname{Red}^{\alpha}(P), \rightarrow\right)$ such that $\operatorname{conc}\left(r^{\prime}\right)=\psi$ and $\rightarrow \operatorname{Strip}\left(\operatorname{Red}^{\alpha}(P), \rightarrow\right) \vDash \operatorname{prem}\left(r^{\prime}\right)$. Then, there is a rule $r \in R$ such that

$$
r^{\prime}=\frac{\left\{\psi^{\prime} \in \operatorname{pprem}(r) \mid \cup_{\beta<\alpha} \rightarrow_{\operatorname{True}\left(\operatorname{Red} d^{\beta}(P)\right)} \not \neq \psi^{\prime}\right\}}{\operatorname{conc}(r)}
$$

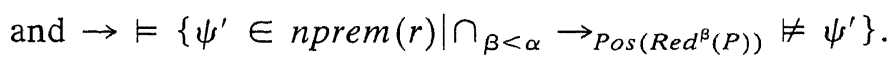

$\rightarrow \vDash n$ nprem $(r)$ : Let $\psi^{\prime} \in \operatorname{nprem}(r)$. If $\cap_{\beta<\alpha} \rightarrow_{\operatorname{Pos}\left(\operatorname{Red}^{\beta}(P)\right)} \not \psi^{\prime}$, then $\rightarrow \vDash$ $\psi^{\prime}$. If $\cap_{\beta<\alpha} \rightarrow_{\operatorname{Pos}\left(\operatorname{Red}^{\beta}(P)\right)} \vDash \psi^{\prime}$, then also $\rightarrow \vDash \psi^{\prime}$, as $\rightarrow \subseteq \cap_{\beta<\alpha}$ $\rightarrow$ Pos $\left(\operatorname{Red}^{\beta}(P)\right)$.

So in $\operatorname{Strip}(P, \rightarrow)$ we have the rule

$$
r^{\prime \prime}=\frac{\operatorname{pprem}(r)}{\operatorname{conc}(r)}
$$

By induction on the depth of the proof of $\psi$ from $\operatorname{Strip}\left(\operatorname{Red}^{\alpha}(P), \rightarrow\right)$ we may conclude that $\rightarrow_{\operatorname{strip}(P, \rightarrow)} \vDash \operatorname{prem}\left(r^{\prime}\right)=\left\{\psi^{\prime} \in \operatorname{pprem}(r) \mid \cup_{\beta<\alpha}\right.$ $\left.\rightarrow_{\text {rrue }\left(\operatorname{Red}^{\beta}(P)\right)} \not \neq \psi^{\prime}\right\}$. By the induction on $\alpha$ and Lemma 5.5, $\forall \beta<\alpha$ : $\rightarrow_{\text {rrue }\left(\operatorname{Red}^{\beta}(P)\right)} \subseteq \rightarrow_{\operatorname{Strip}\left(\operatorname{Red}^{\beta}(P), \rightarrow\right)} \subseteq \rightarrow_{\operatorname{Strip}(P, \rightarrow)}$. Hence, if $\cup_{\beta<\alpha}$ $\rightarrow_{\text {True }\left(\operatorname{Red}^{\beta}(P)\right)} \vDash \psi^{\prime}$, then $\rightarrow_{\operatorname{Strip}(P, \rightarrow)} \vDash \psi^{\prime}$. Thus $\rightarrow_{\operatorname{Strip}(P, \rightarrow)} \vDash \operatorname{prem}\left(r^{\prime \prime}\right)=$ $\operatorname{prem}\left(\boldsymbol{r}^{\prime}\right) \cup\left\{\psi^{\prime} \in \operatorname{pprem}(r) \mid \cup_{\beta<\alpha} \rightarrow_{\text {True }\left(\operatorname{Red}^{\beta}(P)\right)} \vDash \psi^{\prime}\right\}$. So $\rightarrow_{\operatorname{Strip}(P, \rightarrow)} \vDash$ $\operatorname{conc}\left(r^{\prime \prime}\right)=\psi$. 
Our hope is that after sufficiently many reductions we obtain a positive TSS. If this is the case, then our method has succeeded: the transition relation of this positive TSS is the unique transition relation that is stable for the original one. (Example 8.14 shows that the converse is not true: a TSS having a unique stable transition relation need not reduce to a positive TSS.)

Theorem 5.7 (Soundness of Reduction). Let $P=(\Sigma, A, R)$ be a TSS and let $\rightarrow \subseteq \operatorname{Tr}(\Sigma, A)$. For all ordinals $\alpha$ we have:

$$
\rightarrow \text { is stable for } P \Leftrightarrow \rightarrow \text { is stable for } \operatorname{Red}^{\alpha}(P) \text {. }
$$

PROOF

$\Rightarrow$ Let $\rightarrow=\rightarrow_{\operatorname{Strip}(P, \rightarrow)}$.

We prove by induction that for all ordinals $\alpha$ :

$$
\begin{gathered}
\rightarrow=\rightarrow_{\operatorname{Strip}\left(\operatorname{Red}^{\alpha}(P), \rightarrow\right)}, \\
\rightarrow_{\text {True }(\operatorname{Red} \alpha(P))} \subseteq \rightarrow \subseteq \rightarrow \rightarrow_{\operatorname{Pos}\left(\operatorname{Red}^{\alpha}(P)\right)} .
\end{gathered}
$$

By Lemma 5.5, always (1) $\Rightarrow(2)$, so we must prove (1).

Basis. $\rightarrow=\rightarrow_{\operatorname{Strip}(P, \rightarrow)}=\rightarrow_{\operatorname{Strip}\left(\operatorname{Red}^{0}(P), \rightarrow\right)}$ is given.

Induction. By induction, it follows from (2) that for all $\beta<\alpha$ :

$$
\begin{aligned}
& \rightarrow_{\text {True }\left(\operatorname{Red}^{\beta}(P)\right)} \subseteq \rightarrow \subseteq \rightarrow_{P o s\left(\operatorname{Red} d^{\beta}(P)\right)} \text {, so } \\
& \left.\cup_{\beta<\alpha} \rightarrow_{\text {True }(\operatorname{Red} \beta}^{\beta}(P)\right) \subseteq \rightarrow \subseteq \cap_{\beta<\alpha} \rightarrow_{P o s(\operatorname{Red}(P))} \text {, so by Lemma } 5.6 \\
& \rightarrow=\rightarrow_{\operatorname{Strip}(P, \rightarrow)}=\rightarrow_{\operatorname{Strip}\left(\operatorname{Red}^{\alpha}(P), \rightarrow\right)} .
\end{aligned}
$$

$\Leftrightarrow$ Let $\rightarrow=\rightarrow_{\operatorname{Strip}\left(\operatorname{Red}^{\alpha}(P), \rightarrow\right)}$.

Then by Lemma 5.5 for all $\beta<\alpha$ : $\rightarrow_{\text {True }\left(\operatorname{Red}^{\beta}(P)\right)} \subseteq \rightarrow \subseteq_{\operatorname{Pos}\left(\operatorname{Red}^{\beta}(P)\right)}$. So again

$$
\bigcup_{\beta<\alpha} \rightarrow_{\text {True }(\operatorname{Red}(P))} \subseteq \rightarrow \subseteq \underset{\beta<\alpha}{\bigcap} \rightarrow_{\operatorname{Pos}(\operatorname{Red}(P))}
$$

and by Lemma $5.6 \rightarrow=\rightarrow_{\operatorname{Strip}(P, \rightarrow)}=\rightarrow_{\operatorname{Strip}\left(\operatorname{Red}^{\alpha}(P), \rightarrow\right)}$.

Corollary 5.8 (CF. VAN Gelder ET Al. [1988, Corollary 6.2]). If $P$ reduces to a positive TSS, that is, $\operatorname{Red}^{\alpha}(P)$ is positive for some $\alpha$, then $\rightarrow_{\operatorname{Red} d^{\alpha}(P)}$ is associated with $P$.

\section{Reduction and stratification}

We now have two independent methods for associating a transition relation with a TSS with negative premises: reduction and stratification. Three questions arise:

- if both methods are applicable, is their result the same?

- is one method (strictly) stronger than the other?

- is it useful to combine the two methods?

In this section, we shall answer these questions affirmatively. We show that for a stratified TSS $P$, the relation $\rightarrow_{P}$ as defined in Section 3 is stable for $P$. 
Furthermore, we show that repeatedly reducing a stratified TSS yields a positive TSS. Thus, $\rightarrow_{P}$ is the unique transition relation that is stable for $P$. This is also the answer to our second question: reduction is indeed stronger than stratification (that it is strictly stronger is easily seen by the second TSS in Remark 4.4).

So it seems that there is no point in combining the two methods: the result could not be stronger than reduction alone. However, for practical purposes the combination appears to be valuable, due to the fact that the existence of a stratification is generally easier to demonstrate. Therefore, we show in this section that the methods can be used cooperatively, rather than being alternatives for each other.

Finally, we use this amalgamation to demonstrate that the TSS BPA $\delta \epsilon \tau$ with priorities has an associated transition relation under some conditions.

THEOREM 6.1. If $P$ is stratified, then $\rightarrow_{P}$ is stable for $P$.

Proof. Let $P=(\Sigma, A, R)$ and let $S: \operatorname{Tr}(\Sigma, A) \rightarrow \alpha$ be a stratification of $P$.

(1) We show that $\rightarrow_{\operatorname{Strip}\left(P, \rightarrow_{P}\right)} \subseteq \rightarrow_{P}$. Suppose $\operatorname{Strip}\left(P, \rightarrow_{P}\right) \vdash \psi$. We use induction on the structure of the proof of $\psi$. As $\operatorname{Strip}\left(P, \rightarrow_{P}\right) \vdash \psi$, there exists a rule $r^{\prime} \in \operatorname{Strip}\left(R, \rightarrow_{P}\right)$ such that prem $\left(r^{\prime}\right) \subseteq \rightarrow_{\operatorname{Strip}\left(P, \rightarrow_{P}\right)}$ and $\psi=$ $\operatorname{conc}\left(r^{\prime}\right)$. So $\exists r \in R: \operatorname{pprem}(r)=\operatorname{prem}\left(r^{\prime}\right), \operatorname{conc}(r)=\operatorname{conc}\left(r^{\prime}\right)$ and $\rightarrow_{P}$ $\vDash \operatorname{nprem}(r)$. By induction pprem $(r) \subseteq \rightarrow_{P}$. Hence, $\rightarrow_{P} \vDash \operatorname{prem}(r)$. As by Theorem 3.16, $\rightarrow_{P}$ is a model of $P, \psi=\operatorname{conc}(r) \in \rightarrow_{P}$.

(2) Here, it is shown that $\rightarrow_{P} \subseteq \rightarrow_{\operatorname{Strip}\left(P, \rightarrow_{P}\right)}$. Recall that $\rightarrow_{P}=\cup_{0 \leq i<\alpha} \rightarrow_{P_{i}}$. We show by induction that for every $i, 0 \leq i<\alpha: \rightarrow_{P_{i}} \subseteq \rightarrow_{\operatorname{Strip}\left(P, \rightarrow_{P}\right)}$. Let $\psi \in \rightarrow_{P_{i}}$; hence, $P_{i} \vdash \psi$. With induction on the proof of $\psi$ from $P_{i}$ we show that $\operatorname{Strip}\left(P, \rightarrow_{P}\right) \vdash \psi$. Suppose the last rule used to prove $\psi$ from $P_{i}$ is $r^{\prime}$. This means, according Definition 3.14, that there is a rule $r \in R$ and a substitution $\sigma: V \rightarrow T(\Sigma)$ such that

$$
\begin{gathered}
\underset{0 \leq j<i}{\cup} \rightarrow_{P_{j}} \vDash \operatorname{nprem}(\sigma(r)) \cup\{\varphi \in \operatorname{pprem}(\sigma(r)) \mid S(\varphi)<i\}, \\
r^{\prime}=\frac{\{\varphi \in \operatorname{pprem}(\sigma(r)) \mid S(\varphi)=i\}}{\operatorname{conc}(\sigma(r))}
\end{gathered}
$$

and $\operatorname{conc}(r)=\psi$. As $P$ is stratified, for all $t \stackrel{a}{\rightarrow} \in \operatorname{nprem}(\sigma(r))$ and $t^{\prime} \in T(\Sigma): S\left(t \stackrel{a}{\rightarrow} t^{\prime}\right)<S(\psi)=i$. Thus, $\cup_{0 \leq j<i} \rightarrow_{P_{j}} \vDash n$ prem $(\sigma(r))$ implies $\rightarrow_{P} \vDash \operatorname{nprem}(\sigma(r))$ and therefore there is a rule

$$
r^{\prime \prime}=\frac{\operatorname{pprem}(\sigma(r))}{\operatorname{conc}(\sigma(r))} \in \operatorname{Strip}\left(P, \rightarrow_{p}\right) .
$$

For all $\chi \in \operatorname{prem}\left(r^{\prime \prime}\right)$ with $S(\chi)<i: \rightarrow_{P_{S(x)}} \vDash \chi$, so by induction $\rightarrow{\operatorname{Strip}\left(P, \rightarrow_{P}\right)} \vDash \chi$. For all $\chi \in \operatorname{prem}\left(r^{\prime \prime}\right)$ with $S(\chi)=i$, it follows with induction on the proof tree that $\rightarrow_{\operatorname{Strip}\left(P, \rightarrow_{P}\right)} \vDash \chi$. Hence, $\operatorname{Strip}\left(P, \rightarrow_{P}\right)$ $\vdash \operatorname{prem}\left(r^{\prime \prime}\right)$ and hence, $\operatorname{Strip}\left(P, \rightarrow_{P}\right) \vdash \operatorname{conc}\left(r^{\prime \prime}\right)=\psi$.

THEOREM 6.2. Let $P=(\Sigma, A, R)$ be a TSS with stratification $S: \operatorname{Tr}(\Sigma, A) \rightarrow \alpha$. Then $\operatorname{Red}^{\alpha}(P)$ is a positive TSS. 
PROOF. We show that $\cup_{\beta<\alpha} \rightarrow_{\text {True }\left(\operatorname{Red}^{\beta}(P)\right)^{*}}=\cap_{\beta<\alpha} \rightarrow_{P o s\left(\operatorname{Red}^{\beta}(P)\right)}$. According to Remark 5.2, this is sufficient.

$\subseteq$. This implication follows immediately from Lemma 5.5.

․ We claim that for any $\psi \in \operatorname{Tr}(\Sigma, A)$ :

$$
\psi \in \rightarrow_{P o s(\operatorname{Red} S(\psi)(P))} \Rightarrow \psi \in \rightarrow_{\text {True }\left(\operatorname{Red}^{S(\psi)}(P)\right)} .
$$

Using the claim, we can easily finish the proof: as $S(\psi)<\alpha$, we have

$$
\begin{aligned}
& \psi \in \cap_{\beta<\alpha} \rightarrow_{P o s(\operatorname{Red}(P))} \Rightarrow \\
& \psi \in \rightarrow_{\operatorname{Pos}(\operatorname{Red} S(\psi)(P))} \Rightarrow \\
& \psi \in \rightarrow_{\operatorname{True}\left(\operatorname{Red}^{S(\psi)}(P)\right)} \Rightarrow \\
& \psi \in \cup_{\beta<\alpha} \rightarrow_{\operatorname{True}\left(\operatorname{Red}^{S}(\psi)(P)\right)} .
\end{aligned}
$$

We prove our claim by transfinite induction on $S(\psi)$. Assume the induction hypothesis holds for all $\gamma<\beta$. Take some $\psi \in \operatorname{Tr}(\Sigma, A)$ with $S(\psi)=\beta$. Furthermore, assume $\psi \in \rightarrow_{\operatorname{Pos}\left(\operatorname{Red}^{\beta}(P)\right)}$. Hence, there is a proof of $\psi$ from $\operatorname{Pos}\left(\operatorname{Red}^{\beta}(P)\right)$. With induction on this proof, we show that $\operatorname{True}\left(\operatorname{Red}^{\beta}(P)\right) \vdash$ $\psi$. As $\operatorname{Pos}\left(\operatorname{Red}^{\beta}(P)\right) \vdash \psi$, there is a rule $r \in \operatorname{Pos}\left(\operatorname{Red}^{\beta}(R)\right)$ such that $\operatorname{conc}(r)=\psi$ and $\operatorname{Pos}\left(\operatorname{Red}^{\beta}(P)\right) \vdash \operatorname{prem}(r)$. Hence, there is some rule $r^{\prime} \in$ $\operatorname{Red}^{\beta}(R)$ such that $\operatorname{conc}\left(r^{\prime}\right)=\operatorname{conc}(r)=\psi$ and $\operatorname{pprem}\left(r^{\prime}\right)=\operatorname{prem}(r)$. We show that $\operatorname{nprem}\left(r^{\prime}\right)=\emptyset$. In order to obtain a contradiction, assume $t \stackrel{a}{\rightarrow} \in \operatorname{nprem}\left(r^{\prime}\right)$. As $r^{\prime} \in \operatorname{Red}^{\beta}(P)$, we know:

$$
\bigcup_{\zeta<\beta} \rightarrow_{\text {True }(\operatorname{Red}(P))} \vDash t \stackrel{a}{\rightarrow} .
$$

So for every $t^{\prime} \in T(\Sigma): t \stackrel{a}{\rightarrow} t^{\prime} \notin \cup_{\zeta<\beta} \rightarrow_{\text {True }\left(\operatorname{Red}^{\zeta}(P)\right)}$. In particular, as $S\left(t \stackrel{a}{\rightarrow} t^{\prime}\right)=\gamma^{\prime}<S(\psi)=\beta, t \stackrel{a}{\rightarrow} t^{\prime} \notin \stackrel{\rightarrow}{\rightarrow}_{\text {True }\left(\operatorname{Red}^{\gamma^{\prime}}(P)\right)}$. By induction, $t \stackrel{a}{\rightarrow} t^{\prime} \notin$ $\rightarrow_{P o s\left(\operatorname{Red}^{\gamma^{\prime}}(P)\right)}$ and so $t \stackrel{a}{\rightarrow} t^{\prime} \notin \cap_{\zeta<\beta} \rightarrow_{\operatorname{Pos}\left(\operatorname{Red}^{\zeta}(P)\right)}$. Therefore,

$$
\bigcap_{\zeta<\beta} \rightarrow_{P o s(\operatorname{Red} \zeta(P))} \vDash t \stackrel{a}{\rightarrow} \text {. }
$$

Hence, $t^{a} \rightarrow \notin \operatorname{nprem}\left(r^{\prime}\right)$. As nprem $\left(r^{\prime}\right)=\emptyset, r=r^{\prime} \in \operatorname{True}\left(\operatorname{Red}^{\beta}(R)\right)$. By induction (on the depth of the proof tree of $\operatorname{Pos}\left(\operatorname{Red}^{\beta}(P)\right) \vdash \psi$ ), we know that $\operatorname{True}\left(\operatorname{Red}^{\beta}(P)\right) \vdash \operatorname{prem}(r)$ and thus $\operatorname{True}\left(\operatorname{Red}^{\beta}(P)\right) \vdash \psi$. So we can conclude $\psi \in \rightarrow_{\text {True }\left(\operatorname{Red}^{\beta}(P)\right)}$.

Corollary 6.3 (CF. Gelfond AND Lifschitz [1988, Corollary 1] AND VAN Gelder et Al. [1988, TheOREM 6.3]). Let $P=(\Sigma, A, R)$ be a TSS with stratification $S: \operatorname{Tr}(\Sigma, A) \rightarrow \alpha$. Then $\rightarrow_{P}=\rightarrow_{\operatorname{Red}^{\alpha}(P)}$ is associated with $P$.

Proof. Directly using Theorem 6.1, Theorem 6.2, and Corollary 5.8.

Lemma 6.4. Let $P$ be a TSS.

$$
\operatorname{Red}^{\alpha}\left(\operatorname{Red}^{\beta}(P)\right)=\operatorname{Red}^{\alpha+\beta}(P) .
$$

Proof. Straightforward with induction on $\alpha$, using Lemma 5.5. 
Corollary 6.5 (COMBIning Reduction And Stratification). Let $P=$ $(\Sigma, A, R)$ be a TSS and suppose that for some ordinals $\alpha$ and $\beta, S: \operatorname{Tr}(\Sigma, A) \rightarrow \alpha$ is a stratification of $\operatorname{Red}^{\beta}(P)$. Then $\operatorname{Red}^{\alpha+\beta}(P)$ is a positive TSS and $\rightarrow_{\operatorname{Red}^{\alpha+\beta}(P)}=$ $\rightarrow_{\operatorname{Red}^{\beta}(P)}$ is associated with $P$.

Proof. By Theorem 6.2 and Lemma 6.4 it follows that $\operatorname{Red}^{\alpha}\left(\operatorname{Red}^{\beta}(P)\right)=$ $\operatorname{Re}^{\alpha+\beta}(P)$ is a positive TSS. Using Corollary 6.3 and Lemma 6.4 we have that

$$
\rightarrow_{\operatorname{Red} \beta(P)}=\rightarrow_{\operatorname{Red}^{\alpha}(\operatorname{Red} \beta(P))}=\rightarrow_{\operatorname{Red}^{\alpha+\beta}(P)}
$$

is the transition relation associated with $\operatorname{Red}^{\beta}(P)$. Now by Theorem $5.7 \rightarrow \operatorname{Red}^{\beta}(P)$ is associated with $P$.

In the remainder of this section, we apply this corollary to show that a transition relation is associated with an instance $P_{\theta}$ of $\mathrm{BPA}_{\delta \epsilon \tau}$ with priorities, provided that two conditions hold:

(1) The abstraction operator $\tau_{I}$ does not occur in process terms. The reason for this condition was already shown in Example 4.8. This is standard practice in process algebra.

(2) There is no $a \in A c t$ such that $\tau<a$. The motivation for this second condition is threefold (cf. Vaandrager [1990] where it is argued that $\tau>a$ for all actions $a$ seems the most 'intuitive' choice).

- It is essential that $\tau$-actions are not observable. Thus between two observable actions, any number of $\tau$-actions can take place, and must be possible in any process specification. Indeed, the $\tau$-rules R9.1-R9.3 ensure that (in $\mathrm{BPA}_{\delta \epsilon \tau}$ ) every specification satisfies this property. However, allowing $\tau<a$ would destroy this property, as in this case, that is, $\theta(a \cdot a)$ specifies a process performing two $a$-actions, with no $\tau$-actions in between (assuming there is no $b>a$ ):

$$
\operatorname{True}\left(P_{\theta}\right) \vdash \theta(a \cdot a) \stackrel{a}{\rightarrow} \theta(\epsilon \cdot a) \stackrel{a}{\rightarrow} \theta(\epsilon),
$$

but for no $t, t^{\prime} \in T\left(\Sigma_{\theta}\right)$ :

$$
\operatorname{Pos}\left(\operatorname{Red}^{1}\left(P_{\theta}\right)\right) \vdash \theta(a \cdot a) \stackrel{a}{\rightarrow} t \stackrel{\tau}{\rightarrow} t^{\prime} .
$$

(If $\theta(a \cdot a) \stackrel{a}{\rightarrow} t \in \rightarrow_{P o s\left(\operatorname{Red}^{1}\left(P_{\theta}\right)\right)}$, then $t \equiv \theta(u)$ for some $u$ such that $\operatorname{True}\left(P_{\theta}\right) \vdash u \stackrel{a}{\rightarrow} \epsilon$; every rule in $P_{\theta}$ with a conclusion of the form $\theta(u) \stackrel{\tau}{\rightarrow} t^{\prime}$ has a premise $\theta(u) \stackrel{\tau}{\rightarrow} u^{\prime}\left(\mathrm{R} 9.2\right.$ and R9.3) or $u^{a} \rightarrow$ (R5.1)).

- As a consequence, the axiom $\theta(a \cdot x)=a \cdot \theta(x)$, which is part of the complete axiomatization of $\mathrm{BPA}_{\epsilon \delta}$ with priorities (without $\tau$, Cf. Baeten et al. [1986] axiom TH1 and TH2), is no longer valid: when $\tau<a, \theta(a \cdot a)$ cannot perform $\tau$ after $a$ although $a \cdot \theta(a)$ can.

- We conjecture that there is only one transition relation stable for $P_{\theta}$, even for instances with $\tau<a$. However, we have no proof for this. In particular, we do not know whether such an instance of $\mathrm{BPA}_{\delta \epsilon \tau}$ with priorities always reduces to a stratified TSS. The problem is caused by the fact that we do not reduce one TSS (with $(A c t,<)$ and $(\Xi, E)$ fixed), but 
try to reduce the whole class of instances of $\mathrm{BPA}_{\delta \epsilon \tau}$ with priorities (satisfying condition 1) at once.

THEOREM 6.6. If for all $\left(X \Leftarrow t_{X}\right) \in E: \tau_{I}(\cdot)$ does not occur in $t_{X}$ and for all $a \in$ Act it does not hold that $\tau<a$, then there is a transition relation associated with $P_{\theta}$.

Proof. We show that $P_{\theta}$ is stratified after one reduction step. To this end we formulate a useful property of $\operatorname{Red}^{1}\left(P_{\theta}\right)$. Define $N: T\left(\Sigma_{\theta}\right) \rightarrow \mathbf{N}$ as the depth of nestings of $\tau_{I}(\cdot) \mathrm{s}$ by:

$$
\begin{aligned}
& N(a)=N(\epsilon)=N(\delta)=N(\tau)=N(X)=0 \quad(X \in \Xi \text { and } a \in A c t), \\
& N(x+y)=N(x \cdot y)=N(x \triangleleft y)=\max (N(x), N(y)), \\
& N(\theta(x))=N(x), \\
& N\left(\tau_{I}(x)\right)=N(x)+1 .
\end{aligned}
$$

We show that the nesting of $\tau_{I}$ s does not increase by performing an action.

FACT 6.7. For all $a \in A_{\theta}$ we have:

$$
t \stackrel{a}{\rightarrow} u \in \rightarrow_{\operatorname{Pos}\left(P_{\theta}\right)} \Rightarrow N(t) \geq N(u) .
$$

PROOF OF FACT 6.7. It can be shown for every ground instance $r$ of a rule in $P_{\theta}$ that if for every literal $t \stackrel{a}{\rightarrow} u \in \operatorname{pprem}(r) N(t) \geq N(u)$ holds, then $N\left(t^{\prime}\right) \geq$ holds, where $\operatorname{conc}(r)=t^{\prime} \stackrel{b}{\rightarrow} u^{\prime}$. Instead of giving a detailed treatment of each rule, we only prove the most important ones here:

R4.1 $N(x \cdot y)=\max (N(x), N(y)) \geq N(x) \geq N\left(x^{\prime}\right)$ and $\max (N(x), N(y)) \geq$ $N(y)$. This implies that $\max (N(x), N(y)) \geq \max \left(N\left(x^{\prime}\right), N(y)\right)=$ $N\left(x^{\prime} \cdot y\right)$.

R8 $N(X)=0$. So we must prove $N(y)=0$. Indeed $N(y) \leq N\left(t_{X}\right)=0$ as by assumption $t_{X}$ does not contain $\tau_{I^{-}}$-operators.

For example, the literal $t \stackrel{b}{\rightarrow} \tau_{\{a\}}(\theta(t))$ used in Example 3.17 to make $t \stackrel{b}{\rightarrow} \tau_{\{a\}}(\theta(u))$ depend negatively on itself is not possible. Based on this definition of $N$ we define the preorder $\leq$ on pairs of literals by:

$$
(t \stackrel{a}{\rightarrow} u) \leq\left(t^{\prime} \stackrel{b}{\rightarrow} u^{\prime}\right) \quad \text { iff }\left\{\begin{array}{l}
N(t)<N\left(t^{\prime}\right) \text { or } \\
N(t)=N\left(t^{\prime}\right) \text { and }(a=\tau, \sqrt{ }), a>b, \text { or } a=b .
\end{array}\right.
$$

For some ordinal $\alpha$ we can now define a function $S: \operatorname{Tr}\left(\Sigma_{\theta}, A_{\theta}\right) \rightarrow \alpha$ obtained by transforming the preorder $\leq$ into a complete well-founded ordering, which is guaranteed as $<$ on natural numbers is well founded and $>$ on actions is backwardly well founded (see Example 2.4):

$$
\begin{aligned}
& \varphi \approx \psi \text { iff } \varphi \leq \psi \text { and } \psi \leq \varphi, \\
& \varphi \approx \psi \Rightarrow S(\varphi)=S(\psi), \\
& \varphi \leq \psi \text { and not } \varphi \approx \psi \Rightarrow S(\psi)<S(\psi) .
\end{aligned}
$$


(We do not need a more precise definition of $S$; as such a definition necessarily depends on the size of the set $A c t$, we omit it).

FACT 6.8. $S$ is a stratification of Reduce $\left(P_{\theta}, \rightarrow_{\text {True }\left(P_{\theta}\right)}, \rightarrow_{\operatorname{Pos}\left(P_{\theta}\right)}\right)$.

PROOF OF FACT 6.8. Let $r$ be a ground instance of a rule in Reduce $\left(P_{\theta}\right.$, $\left.\rightarrow_{\text {True }\left(P_{\theta}\right)}, \rightarrow_{\text {Pos }\left(P_{\theta}\right)}\right)$. We must show that for every $\psi \in \operatorname{pprem}(r): S(\psi) \leq$ $S(\operatorname{conc}(r))$. Furthermore, it must hold that for every $\psi=t^{a} \nrightarrow \in \tan \rightarrow(r)$ and for every $t^{\prime} \in T(\Sigma): S\left(t \stackrel{a}{\rightarrow} t^{\prime}\right)<S(\operatorname{conc}(r))$. For most rules this is trivial, as the unreduced instances of the rule already satisfy the requirement. We only consider the most interesting cases:

R5.1 $N(\theta(x))=N(x)$ implies that $S\left(\theta(x) \stackrel{a}{\rightarrow} \theta\left(x^{\prime}\right)\right)=S\left(x \stackrel{a}{\rightarrow} x^{\prime}\right)$. For each $b>a$ it holds that $S\left(x \stackrel{b}{\rightarrow} t^{\prime}\right)<S\left(x \stackrel{a}{\rightarrow} x^{\prime} ;\right.$ for any $t^{\prime} \in$ $T\left(\Sigma_{\theta}\right)$.

R6.1 $N(x) \leq N(x \triangleleft y)$, so $S\left(x \stackrel{a}{\rightarrow} x^{\prime}\right) \leq S\left(x \triangleleft y \stackrel{a}{\rightarrow} x^{\prime}\right)$. Also $N(y) \leq$ $N(x \triangleleft y)$, so for each $b>a$ and $t^{\prime} \in T\left(\Sigma_{\theta}\right): S\left(y \stackrel{b}{\rightarrow} t^{\prime}\right)$ $<S\left(x \triangleleft y \stackrel{a}{\rightarrow} x^{\prime}\right)$.

R7.2 $N\left(\tau_{I}(x)\right)=N(x)+1>N(x)$. So $S\left(x \stackrel{a}{\rightarrow} x^{\prime}\right)<S\left(\tau_{I}(x) \stackrel{\tau}{\rightarrow} \tau_{I}\left(x^{\prime}\right)\right)$.

R9.2 and R9.3 By the first fact $\operatorname{Reduce}\left(P_{\theta}, \rightarrow_{\operatorname{True}\left(P_{\theta}\right)}, \rightarrow_{\operatorname{Pos}\left(P_{\theta}\right)}\right)$ contains only those instances of these rules for which $N(x) \geq N(y) \geq N(z)$. We need $N(x) \geq N(y)$ to prove, for example, $(y \stackrel{\tau}{\rightarrow} z)$ $\leq(x \stackrel{a}{\rightarrow} z)$, hence $S(y \stackrel{\tau}{\rightarrow} z) \leq S(x \stackrel{a}{\rightarrow} z)$.

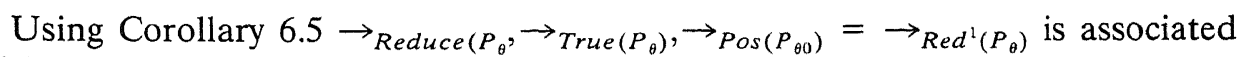
with $P_{\theta}$.

\section{Bisimulation relations}

We have defined the meaning of a TSS as its associated transition relation and shown how to arrive at this transition relation. Now we switch to the study of properties of transition relations as consequences of properties of their defining TSSs.

An important question (for example, in process verification) is whether two terms denote the 'same' process. Many process equivalences based on transition relations have been proposed [van Glabbeek 1990; 1993b], of which strong bisimulation equivalence is often used [Milner 1980; Park 1981]. In this and the subsequent sections, some relations between TSSs and strong bisimulation equivalence are studied.

Definition 7.1. Let $P$ be a TSS with associated transition relation $\rightarrow_{P}$. A relation $R$ is a strong bisimulation relation based on $P$ if it satisfies:

- whenever $t R u$ and $t \stackrel{a}{\rightarrow}{ }_{p} t^{\prime}$, then, for some $u^{\prime} \in T(\Sigma)$, we have $u \stackrel{a}{\rightarrow}{ }_{P} u^{\prime}$ and $t^{\prime} R u^{\prime}$,

- whenever $t R u$ and $u \stackrel{a}{\rightarrow}{ }_{P} u^{\prime}$, then, for some $t^{\prime} \in T(\Sigma)$, we have $t \stackrel{a}{\rightarrow}_{P} t^{\prime}$ and $t^{\prime} R u^{\prime}$.

Two terms $t, u \in T(\Sigma)$ are $(P-)$ bisimilar, notation $t \leftrightarrow \leftrightarrow_{P} u$, if there is a strong bisimulation relation $R$ based on $P$ such that $t R u$. Note that $\leftrightarrow_{P}$, the strong bisimulation equivalence induced by $P$, is an equivalence relation. 
In the next section, we prove that under specific conditions on $P, \leftrightarrow_{P}$ is a congruence relation. To this end we shall approximate $\rightarrow_{P}$ by other transition relations $\rightarrow_{Q}$, and use the notion of $P \Rightarrow Q$-bisimulation, meaning that if $t$ can do some step in $\rightarrow_{P}, u$ can do a 'similar' step in $\rightarrow_{Q}$ (and vice versa, i.e. if $u$ can do a step in $\rightarrow_{P}, t$ can do a 'similar' step in $\rightarrow_{Q}$ ). In the end, the approximation $\rightarrow_{Q}$ will be equal to $\rightarrow_{p}$. It may be readily checked that in this case, $P \Rightarrow Q$ bisimulation is exactly $P$-bisimulation. Thus showing that for every approximation $\rightarrow_{Q} P \Rightarrow Q$-bisimulation is a congruence is sufficient to show that $P$ bisimulation is a congruence.

Formally, we have the following definition.

Definition 7.2. Let $P=\left(\Sigma, A, R_{P}\right)$ and $Q=\left(\Sigma, A, R_{Q}\right)$ be TSSs with associated transition relations $\rightarrow_{P}$ and $\rightarrow_{Q}$. A relation $R$ is a strong $P \Rightarrow Q$ bisimulation relation if it satisfies:

- whenever $t R u$ and $t \stackrel{a}{\rightarrow}_{P} t^{\prime}$, then, for some $u^{\prime} \in T(\Sigma)$, we have $u \stackrel{a}{\rightarrow} Q u^{\prime}$ and $t^{\prime} R u^{\prime}$,

- whenever $t R u$ and $u \stackrel{a}{\rightarrow}_{P} u^{\prime}$, then, for some $t^{\prime} \in T(\Sigma)$, we have $t \stackrel{a}{\rightarrow}_{Q} t^{\prime}$ and $t^{\prime} R u^{\prime}$.

We say that two terms $t, u \in T(\Sigma)$ are $P \Rightarrow Q$-bisimilar, notation $t \leftrightarrow_{P \Rightarrow Q} u$, if there is a strong $P \Rightarrow Q$-bisimulation relation $R$ such that $t R u$. Note that like $\leftrightarrow_{P}$, $\leftrightarrow_{P \Rightarrow Q}$ is symmetric. In contrast with $\leftrightarrow_{P}, \leftrightarrow_{P \Rightarrow Q}$ need not be transitive or reflexive.

\section{The ntyft/ntyxt-format and the Congruence Theorem}

A desirable property for TSSs is that the induced strong bisimulation equivalence is a congruence. In Groote and Vaandrager [1992], this led to the observation that if a (positive) TSS is in the so-called tyft/tyxt-format then this is the case. In Groote [1993], this result was extended to stratified TSSs. In order to express the fact that negative premises are allowed, $n$ s were added to the name of the format, obtaining the ntyft/ntyxt-format. In this section we show that even for TSSs that are positive after reduction, bisimulation is a congruence if the TSS is in ntyft/ntyxt-format. In the end of this section we show that positive after reduction' is a necessary requirement for the Congruence Theorem: we give a TSS in ntyft/ntyxt-format with a unique stable transition relation for which strong bisimulation is not a congruence.

Definition 8.1. Let $\Sigma=(F$, rank $)$ be a signature. Let $P=(\Sigma, A, R)$ be a TSS. A rule $r \in R$ is in ntyft-format if it has the form:

$$
\frac{\left\{t_{k} \stackrel{a_{k}}{\rightarrow} y_{k} \mid k \in K\right\} \cup\left\{t_{l}^{b_{l}} \rightarrow|l| l \in L\right\}}{f\left(x_{1}, \ldots, x_{\operatorname{rank}(f)}\right) \stackrel{a}{\rightarrow} t}
$$

with $K$ and $L$ (possibly infinite) index sets, $y_{k}, x_{i}(1 \leq i \leq \operatorname{rank}(f))$ all different variables, $a_{k}, b_{l}, a \in A, f \in F$ and $t_{k}, t_{l}, t \in \mathbb{T}(\Sigma)$. A rule $r \in R$ is in ntyxt-format if it fits: 


$$
\frac{\left\{t_{k} \stackrel{a_{k}}{\rightarrow} y_{k} \mid k \in K\right\} \cup\left\{t_{l}^{b_{l}} \rightarrow \mid l \in L\right\}}{x \stackrel{a}{\rightarrow} t}
$$

with $K, L$ (possibly infinite) index sets, $y_{k}, x$ all different variables, $a_{k}, b_{l}, a \in$ $A, t_{k}, t_{l}$ and $t \in \mathbb{T}(\Sigma) . P$ is in ntyft-format if all its rules are in $n t y f t$-format and $P$ is in $n t y f t / n t y x t$-format if all its rules are either in $n t y f t$ - or in $n t y x t$-format.

It may be useful to point out why this format is called the ntyft/ntyxt-format. As stated above, the ' $n$ ' was added to indicate the possibility of negative premises. The letters tyft can be found if one reads first the (positive) premises and then the conclusion from left to right: $t$ represents a term in the left hand side of a premise, $y$ the variable in the right hand side; $f$ is the function name in the left hand side of the conclusion and $t$ the term in the right-hand side. Similarly, the other format is called ntyxt.

As in Groote [1993] and Groote and Vaandrager [1992], we use a wellfoundedness condition in order to prove the Congruence Theorem. However, this restriction is not essential, as it is proven in Fokkink and van Glabbeek [1995] that every transition system in ntyft/ntyxt-format that is positive after reduction can be transformed into an equivalent well-founded transition system in the same format which is also positive after reduction.

Definition 8.2 (Well-foundedness). Let $P=(\Sigma, A, R)$ be a TSS. Let $S=\left\{t_{k} \stackrel{a_{k}}{\rightarrow} t_{k}^{\prime} \mid k \in K\right\} \subseteq \mathbb{T}(\Sigma) \times A \times \mathbb{T}(\Sigma)$ be a set of positive literals over $\Sigma$ and $A$. The variable dependency graph of $S$ is a directed (unlabeled) graph $V D G$ with:

-Nodes: $\cup_{k \in K} \operatorname{Var}\left(t_{k} \stackrel{a_{k}}{\rightarrow} t_{k}^{\prime}\right)$,

-Edges: $\left\{\langle x, y\rangle \mid x \in \operatorname{Var}\left(t_{k}\right), y \in \operatorname{Var}\left(t_{k}^{\prime}\right)\right.$ for some $\left.k \in K\right\}$.

$S$ is called well founded if any backward chain of edges in the variable dependency graph is finite. A rule is called well founded if its set of positive premises is well founded. A TSS is called well founded if all its rules are well founded.

Definition 8.3. Let $P=(\Sigma, A, R)$ be a TSS. Let $r \in R$ be a rule. A variable $x$ is called free in $r$ if it occurs in $r$ but not in the source of the conclusion or in the target of a positive premise. The rule $r$ is called pure if it is well founded and does not contain free variables. $P$ is called pure if all rules in $R$ are pure.

In what follows, we state a number of technicalities needed for the proof of Theorem 8.13. At first reading it is advised to skip the remainder of this section except for this theorem.

Definition 8.4. Let $S$ be a set of positive literals which is well founded and let $V D G$ be the variable dependency graph of $S$. Let $\operatorname{Var}(S)$ be the set of variables occurring in literals in $S$. Define for each $x \in \operatorname{Var}(S): n_{V D G}(x)=$ $\sup \left(\left\{n_{V D G}(y)+1 \mid\langle y, x\rangle\right.\right.$ is an edge of $\left.\left.V D G\right\}\right)(\sup (\emptyset)=0)$.

Remark 8.5. If $S$ is a set of positive premises of a rule in $n t y f t / n t y x t$-format then $n_{V D G}(x) \in \mathbf{N}$ for each $x \in \operatorname{Var}(S)$ : Every variable $y_{k}$ occurs only once in the right hand side of a positive literal in the premises. As the term $t_{k}$ is finite, it contains only a finite number of variables $x$. Therefore, the set $U=\left\{n_{V D G}(x)+\right.$ 
$1 \mid\left\langle x, y_{k}\right\rangle$ is an edge of $\left.V D G\right\}$ is finite. Hence, $n_{V D G}\left(y_{k}\right)=\sup (U)$ is a natural number.

The following lemma states that any TSS in ntyft/ntyxt-format is 'equivalent' to a pure TSS in $n t y f t$-format. This allows us to only study $n t y f t$-rules.

LEMMA 8.6. Let $P$ be a well founded TSS in ntyft/ntyxt-format and let $\rightarrow$ be the transition relation associated with $P$. Then there is a pure TSS $P^{\prime}$ in ntyft-format such that $\rightarrow$ is also associated with $P^{\prime}$. Moreover, $P^{\prime}$ is positive after reduction iff $P$ is positive after reduction.

Proof. Assume $P=(\Sigma, A, R)$ and $\Sigma=(F$, rank $)$. First we construct a TSS $P^{\prime \prime}=\left(\Sigma, A, R^{\prime \prime}\right)$ which is pure and in $n t y f t / n t y x t$-format. $R^{\prime \prime}$ contains a rule $\sigma(r)$ iff $r$ is a rule in $R$ and $\sigma: V \rightarrow \mathbb{T}(\Sigma)$ is a substitution such that for each variable that is free in $r: \sigma(x) \in T(\Sigma)$ and for each variable $x$ that is not free in $r \sigma(x)$ $=x$. From $P^{\prime \prime}$, we construct $P^{\prime}$ as follows: $P^{\prime}=\left(\Sigma, A, R^{\prime}\right)$ where for each $f \in$ $F$, a rule $\sigma_{f}(r) \in R^{\prime}$ iff $r$ is a rule in $R^{\prime \prime}$ and $\sigma_{f}: V \rightarrow \mathbb{T}(\Sigma)$ is a substitution satisfying:

$$
\begin{aligned}
& \text { if } r \text { is in } n t y f t \text {-format, then } \sigma_{f}(z)=z \quad \text { for all } z \in V \text {, } \\
& \text { if } r \text { is in } n t y x t \text {-format, then } \sigma_{f}(z)=z \\
& \quad \text { for all } z \in V\{x\} \text { and } \sigma_{f}(x)=f\left(z_{1}, \ldots, z_{\operatorname{rank}(f)}\right) \text {. }
\end{aligned}
$$

Here, $z_{i}(1 \leq i \leq \operatorname{rank}(f))$ are variables that do not occur in $r$. It is easy to see that $P^{\prime}$ is a pure TSS in $n t y f t$-format. Observe that the ground instances of the rules in $R, R^{\prime}$, and $R^{\prime \prime}$ are the same. Also note that 'stable for' and 'positive after reduction' are defined with respect to these ground instances. Therefore, $\rightarrow$ is also the unique transition relation stable for $P^{\prime}$ and $P^{\prime \prime}$. Furthermore, $P^{\prime}$ and $P^{\prime \prime}$ are positive after reduction iff $P$ is positive after reduction.

The relation $R_{P}$ that is defined now forms the backbone of all remaining proofs in this section.

Definition 8.7. Let $\Sigma=(F$, rank $)$ be a signature and let $P=(\Sigma, A, R)$ be a TSS with an associated transition relation. The relation $R_{P} \subseteq T(\Sigma) \times T(\Sigma)$ is the minimal relation satisfying:

$-\leftrightarrow_{P} \subseteq R_{P}$

-for all function names $f \in F$ :

$$
\forall 1 \leq k \leq \operatorname{rank}(f): u_{k} R_{P} v_{k} \Rightarrow f\left(u_{1}, \ldots, u_{\operatorname{rank}(f)}\right) R_{P} f\left(v_{1}, \ldots, v_{\operatorname{rank}(f)}\right) .
$$

Note that this definition is in fact saying that $R_{P}$ is the minimal congruence relation that includes $\leftrightarrow_{P}$. This explains the following lemma, which is a standard fact about congruence relations.

LEMMA 8.8. Let $P=(\Sigma, A, R)$ be a TSS with an associated transition relation. Let $t \in T(\Sigma)$ and let $\sigma, \sigma^{\prime}: V \rightarrow T(\Sigma)$ be substitutions such that for all $x$ in $\operatorname{Var}(t)$ $\sigma(x) R_{P} \sigma^{\prime}(x)$. Then, $\sigma(t) R_{p} \sigma^{\prime}(t)$.

Proof. Straightforward with induction on the structure of $t$.

LEMMA 8.9. Let $P$ be a pure TSS in ntyft-format. Suppose that $\rightarrow_{P}$ is the transition relation that is associated with $P$. Then for all ordinals $\alpha \geq 0: R_{P}$ is a 
(1) $P \Rightarrow \operatorname{Pos}\left(\operatorname{Red}^{\alpha}(P)\right)$-bisimulation relation.

(2) $\operatorname{True}\left(\operatorname{Red}^{\alpha}(P)\right) \Rightarrow P$-bisimulation relation.

Proof. Assume $P=(\Sigma, A, R)$ and $\Sigma=(F$, rank $)$. We show the two statements in the lemma by mutual transfinite induction on $\alpha$.

(1) For reasons of symmetry it is enough to show that:

$$
\text { if } u R_{P} v \text { and } \rightarrow_{P} \vDash u \stackrel{a}{\rightarrow} u^{\prime},
$$

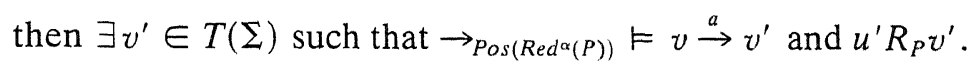

We prove this by induction on the proof of $u \stackrel{a}{\rightarrow} u^{\prime}$ from $\operatorname{Strip}\left(P, \rightarrow_{P}\right)$. As $u R_{P} v$, two cases arise:

$-u \leftrightarrow_{P} v$. Then $\rightarrow_{P} \vDash u \stackrel{a}{\rightarrow} u^{\prime}$ implies $\exists v^{\prime} \in T(\Sigma): \rightarrow_{P} \vDash v \stackrel{a}{\rightarrow} v^{\prime}$ and $u^{\prime} \overleftrightarrow{\leftrightarrow}_{P} v^{\prime}$.

By Lemma 5.5 and Theorem $5.7 \rightarrow_{P} \subseteq \rightarrow_{P o s\left(\operatorname{Red}^{\alpha}(P)\right)}$. So $\rightarrow_{P o s\left(\operatorname{Red}^{\alpha}(P)\right)} \vDash$ $v \stackrel{a}{\rightarrow} v^{\prime}$. Furthermore, $u^{\prime} \overleftrightarrow{\leftrightarrow}_{P} v^{\prime}$ implies $u^{\prime} R_{P} v^{\prime}$.

-For some function name $f \in F, u=f\left(u_{1}, \ldots, u_{\operatorname{rank}(f)}\right), v=f\left(v_{1}, \ldots\right.$, $\left.v_{\operatorname{rank}(f)}\right)$ and $u_{i} R_{P} v_{i}$ for $1 \leq i \leq \operatorname{rank}(f)$. Then there is a rule:

$$
r=\frac{\left\{t_{k} \stackrel{a_{k}}{\rightarrow} y_{k} \mid k \in K\right\} \cup\left\{t_{l}{ }^{a_{l}} f|l| l \mid L\right\}}{f\left(x_{1}, \ldots, x_{\operatorname{rank}(f)}\right) \stackrel{a}{\rightarrow} t} \in R
$$

and a substitution $\sigma$ such that $\sigma\left(x_{i}\right)=u_{i}(1 \leq i \leq \operatorname{rank}(f)), \sigma(t)=u^{\prime}$, $\rightarrow_{P} \vDash \operatorname{prem}(\sigma(r))$ and

$$
\frac{\operatorname{pprem}(\sigma(r))}{\operatorname{conc}(\sigma(r))}
$$

is the last rule of the proof of $u \stackrel{a}{\rightarrow} u^{\prime}$ from $\operatorname{Strip}\left(P, \rightarrow_{P}\right)$. Thus the proof of $\sigma\left(t_{k} \stackrel{a_{k}}{\rightarrow} y_{k}\right)(k \in K)$ from $\operatorname{Strip}\left(P, \rightarrow_{P}\right)$ is less deep. As $P$ is pure, $\left\{x_{1}, \ldots, x_{\operatorname{rank}(f)}\right\} \cup\left\{y_{k} \mid k \in K\right\}=\operatorname{Var}(r)$.

CLAIM 8.10. There is a closed substitution $\sigma^{\prime}$ such that for all $x \in$ $\operatorname{Var}(r)$ :

(a) $\sigma(x) R_{P} \sigma^{\prime}(x)$,

(b) if $x=x_{i}$, then $\sigma^{\prime}(x)=v_{i}$,

(c) if $x=y_{k}(k \in K)$, then $\sigma^{\prime}\left(t_{k} \stackrel{a_{k}}{\rightarrow} y_{k}\right) \in \rightarrow_{\operatorname{Pos}\left(\operatorname{Red}^{\alpha}(P)\right)}$,

(d) for all $l \in L$ and for all $\beta<\alpha: \rightarrow_{\text {True }\left(\operatorname{Red}^{\beta}(P)\right)} \vDash \sigma^{\prime}\left(t_{l}\right)^{a_{l}} \rightarrow$.

PROOF OF CLAIM 8.10. We prove the first three points of the claim by inductively constructing $\sigma^{\prime}(x)$ for every $x \in \operatorname{Var}(r)$, using induction on the degree of $x$ in the VDG of pprem $(r)$.

For $x \in\left\{x_{1}, \ldots, x_{\operatorname{rank}(f)}\right\}, \sigma^{\prime}\left(x_{i}\right)=v_{i}$ is prescribed. Also $\sigma\left(x_{i}\right)=$ $u_{i} R_{P} v_{i}=\sigma^{\prime}\left(x_{i}\right)$ is satisfied.

For $x=y_{k}(k \in K)$, we have $t_{k} \stackrel{a_{k}}{\rightarrow} y_{k} \in \operatorname{pprem}(r)$. For all $y \in \operatorname{Var}\left(t_{k}\right)$, $n_{V D G}(y)<n_{V D G}(x)$, so by induction $\sigma(y) R_{P} \sigma^{\prime}(y)$. As $R_{P}$ is a congruence, $\sigma\left(t_{k}\right) R_{P} \sigma^{\prime}\left(t_{k}\right)$. As the proof of $\sigma\left(t_{k} \stackrel{a_{k}}{\rightarrow} y_{k}\right)$ is less deep than the proof of $u \stackrel{a}{\rightarrow} u^{\prime}$ from $\operatorname{Strip}\left(P, \rightarrow_{P}\right)$, by induction $\exists w \in T(\Sigma): \sigma^{\prime}\left(t_{k}\right) \stackrel{a_{k}}{\rightarrow} w \in$ 
$\rightarrow_{P o s\left(\operatorname{Red}^{\alpha}(P)\right)}$ and $\sigma\left(y_{k}\right) R_{P} w$. Thus, we take $\sigma^{\prime}\left(y_{k}\right)=w$. Note that the first three points of Claim 8.10 are satisfied which finishes the first part of the proof.

It remains to be shown that $\forall \beta<\alpha: \rightarrow_{\operatorname{True}\left(\operatorname{Red}^{\beta}(P)\right)} \vDash$ $\sigma^{\prime}\left(t_{l}\right) \stackrel{a_{l}}{\rightarrow}(l \in L)$. Again $\sigma\left(t_{l}\right) R_{P} \sigma^{\prime}\left(t_{l}\right)$. Assume to generate a contradiction that $\left.\exists \beta<\alpha: \rightarrow_{\text {True }} \operatorname{Red}^{\beta}(P)\right) \vDash \sigma^{\prime}\left(t_{l}\right) \stackrel{a_{l}}{\rightarrow} s$ for some $s$. By simultaneous induction $\forall \beta<\alpha: \sigma\left(t_{l}\right) \overleftrightarrow{\leftrightarrow} \operatorname{True}\left(\operatorname{Red}^{\beta}(P)\right) \Rightarrow_{P} \sigma^{\prime}\left(t_{l}\right)$. So $\rightarrow_{P} \vDash \sigma\left(t_{l}\right) \stackrel{a_{l}}{\rightarrow} s^{\prime}$ for some $s^{\prime}$. This contradicts the fact that $\rightarrow_{P} \vDash \operatorname{prem}(\sigma(r))$. Hence, for all $l \in L$ and for all $\beta<\alpha: \rightarrow_{\operatorname{True}\left(\operatorname{Red}^{\beta}(P)\right)} \vDash \sigma^{\prime}\left(t_{l}\right) \stackrel{a_{l}}{\rightarrow}$.

According to Claim 8.10 , there is a substitution $\sigma^{\prime}$ with the properties (a), (b), (c), and (d).

Consider

$$
\begin{aligned}
& V=\operatorname{Pos}\left(\operatorname{Red}^{\alpha}\left(\left\{\sigma^{\prime}(r)\right\}\right)\right. \\
& =\operatorname{Pos}\left(\operatorname{Reduce}\left(\left\{\sigma^{\prime}(r)\right\}, \underset{\beta<\alpha}{\cup} \rightarrow_{\operatorname{True}(\operatorname{Red} \beta(P))}, \underset{\beta<\alpha}{\bigcap} \rightarrow_{\operatorname{Pos}\left(\operatorname{Red} d^{\beta}(P)\right)}\right)\right) .
\end{aligned}
$$

First we show that $\exists r^{\prime} \in V$. It follows immediately from clause (d) in the claim that

$$
\bigcup_{\beta<\alpha} \rightarrow_{\text {True }(\operatorname{Red} \beta(P))} \vDash n \text { prem }\left(\sigma^{\prime}(r)\right) .
$$

Furthermore, by clause (c) and Lemma 5.5:

$$
\bigcap_{\beta<\alpha} \rightarrow_{P o s(\operatorname{Red}(P))} \vDash \operatorname{pprem}\left(\sigma^{\prime}(r)\right) .
$$

Hence, there is some $r^{\prime} \in V$. It follows from clause (c) in Claim 8.10 that $\rightarrow_{P o s\left(\operatorname{Red}^{\alpha}(P)\right)} \vDash \operatorname{pprem}\left(\sigma^{\prime}(r)\right)$ and therefore, we have that $\rightarrow_{P o s\left(\operatorname{Red}^{\alpha}(P)\right)}$ $\vDash \operatorname{pprem}\left(r^{\prime}\right)=\operatorname{prem}\left(r^{\prime}\right)$. Thus $\rightarrow_{\operatorname{Pos}\left(\operatorname{Red}^{\alpha}(P)\right)} \vDash \operatorname{conc}\left(r^{\prime}\right)=$ $\operatorname{conc}\left(\sigma^{\prime}(r)\right)=v \stackrel{a}{\rightarrow} \sigma^{\prime}(t)$ and $u^{\prime}=\sigma(t) R_{P} \sigma^{\prime}(t)=v^{\prime}$.

(2) For reasons of symmetry it is enough to show that:

$$
\begin{aligned}
& \text { If } u R_{P} v \text { and } \rightarrow_{\text {True }\left(\operatorname{Red}^{\alpha}(P)\right)} \vDash u \stackrel{a}{\rightarrow} u^{\prime}, \\
& \text { then } \exists v^{\prime} \in T(\Sigma) \text { such that } \rightarrow_{P} \vDash v \stackrel{a}{\rightarrow} v^{\prime} \text { and } u^{\prime} R_{P} v^{\prime} .
\end{aligned}
$$

As $\rightarrow_{\text {True }\left(\operatorname{Red}^{\alpha}(P)\right)} \vDash u \stackrel{a}{\rightarrow} u^{\prime}$, there is a proof tree of $u \stackrel{a}{\rightarrow} u^{\prime}$ from True $\left(\operatorname{Red}^{\alpha}(P)\right)$. We use induction on the depth of this proof. As $u R_{P} v$, we can distinguish two cases:

$-u \leftrightarrow_{a} v$. As by Lemma 5.5 and Theorem $5.7 \rightarrow_{\text {True }\left(\operatorname{Red}^{\alpha}(P)\right)} \subseteq \rightarrow_{P}, P \vDash$ $u \stackrel{a}{\rightarrow} u^{\prime}$. So, $\exists v^{\prime} \in T(\Sigma)$ such that $\rightarrow_{p} \vDash v \stackrel{a}{\rightarrow} v^{\prime}$ and $u^{\prime} \stackrel{\leftrightarrow}{\leftrightarrow} v^{\prime}$. Hence, $u^{\prime} R_{p} v^{\prime}$.

-For some function name $f \in F, u=f\left(u_{1}, \ldots, u_{\operatorname{rank}(f)}\right), v=f\left(v_{1}, \ldots\right.$, $\left.v_{\operatorname{rank}(f)}\right)$ and $u_{i} R_{P} v_{i}$ for $1 \leq i \leq \operatorname{rank}(f)$. In this case the final (ground) rule $r \in \operatorname{True}\left(\operatorname{Red}^{\alpha}(R)\right)$ of the proof of $u \stackrel{a}{\rightarrow} u^{\prime}$ from $\operatorname{True}\left(\operatorname{Red}^{\alpha}(P)\right)$ is also present in $\operatorname{Red}^{\alpha}(R)$ and has no negative premises. 


$$
\begin{aligned}
& \operatorname{Red}^{\alpha}(R) \\
& =\left\{\begin{array}{lll}
\operatorname{Reduce}\left(R_{\text {ground }}, \cup_{\beta<\alpha} \rightarrow_{\text {True }\left(\operatorname{Red} d^{\beta}(P)\right)}, \cap_{\beta<\alpha} \rightarrow_{\operatorname{Pos}(\operatorname{Red}(P))}\right) & \text { if } & \alpha>0, \\
R_{\text {ground }} & \text { if } & \alpha=0 .
\end{array}\right.
\end{aligned}
$$

Thus, there is a rule $r^{\prime} \in R$ and a substitution $\sigma: V \rightarrow T(\Sigma)$ such that $\sigma\left(r^{\prime}\right)$ is reduced to $r$. This means that $\operatorname{conc}(r)=\operatorname{conc}\left(\sigma\left(r^{\prime}\right)\right)$ and $\operatorname{prem}(r) \subseteq \operatorname{pprem}\left(\sigma\left(r^{\prime}\right)\right)$. Moreover, all negative premises of $\sigma\left(r^{\prime}\right)$ and all premises in $\operatorname{pprem}\left(\sigma\left(r^{\prime}\right)\right)$ - $\operatorname{pprem}(r)$, which are removed, are redundant:

$$
\begin{aligned}
& \cup_{\beta<\alpha} \rightarrow_{\text {True }(\operatorname{Red}(\mathcal{\beta}))} \vDash \operatorname{pprem}\left(\sigma\left(r^{\prime}\right)\right)-\operatorname{pprem}(r), \\
& \cap_{\beta<\alpha} \rightarrow_{\operatorname{Pos}(\operatorname{Red} \beta(P))} \vDash \operatorname{nprem}\left(\sigma\left(r^{\prime}\right)\right) .
\end{aligned}
$$

As $P$ is in ntyft-format, $r^{\prime}$ is of the form

$$
\frac{\left\{t_{k} \stackrel{a_{k}}{\rightarrow} y_{k} \mid k \in K\right\} \cup\left\{t_{l}^{a_{l}}+f \mid l \in L\right\}}{f\left(x_{1}, \ldots, x_{\operatorname{rank}(f)}\right) \stackrel{a}{\rightarrow} t}
$$

and $\sigma\left(x_{i}\right)=u_{i}(1 \leq i \leq \operatorname{rank}(f))$, hence $\sigma\left(f\left(x_{1}, \ldots, x_{\operatorname{rank}(f)}\right)\right)=u$, and $\sigma(t)=u^{\prime}$. As $P$ is pure, $\left\{x_{1}, \ldots, x_{\operatorname{rank}(f)}\right\} \cup\left\{y_{k} \mid k \in K\right\}=$ $\operatorname{Var}\left(r^{\prime}\right)$.

Claim 8.11. There is a closed substitution $\sigma^{\prime}$ such that for all $x \in \operatorname{Var}\left(r^{\prime}\right)$ :

(a) $\sigma(x) R_{P} \sigma^{\prime}(x)$

(b) if $x=x_{i}$, then $\sigma^{\prime}(x)=v_{i}$,

(c) if $x=y_{k}(k \in K)$, then $\sigma^{\prime}\left(t_{k}\right) \stackrel{a_{k}}{\rightarrow} \sigma^{\prime}\left(y_{k}\right) \in \rightarrow_{P}$,

(d) for all $l \in L: \rightarrow_{P} \vDash \sigma^{\prime}\left(t_{l}\right)^{a_{l}} \rightarrow$.

PROOF OF CLAIM 8.11. We prove the first three points of the claim by giving a construction of $\sigma^{\prime}(x)$ for every $x \in \operatorname{Var}\left(r^{\prime}\right)$, using induction on the degree of $x$ in the VDG of pprem $\left(r^{\prime}\right)$.

For $x \in\left\{x_{1}, \ldots, x_{\operatorname{rank}(f)}\right\}, \sigma^{\prime}\left(x_{i}\right)=v_{i}$ is prescribed. Also $\sigma\left(x_{i}\right)=$ $u_{i} R_{P} v_{i}=\sigma^{\prime}\left(x_{i}\right)$ is satisfied.

For $x=y_{k}(k \in K)$, we have $t_{k} \stackrel{a_{k}}{\rightarrow} y_{k} \in \operatorname{pprem}\left(r^{\prime}\right)$. For all $y \in \operatorname{Var}\left(t_{k}\right)$, $n_{V D G}(y)<n_{V D G}(x)$, so by induction $\sigma(y) R_{P} \sigma^{\prime}(y)$. As $R_{P}$ is a congruence, $\sigma\left(t_{k}\right) R_{P} \sigma^{\prime}\left(t_{k}\right)$. Two cases arise.

(i) $\sigma\left(t_{k} \stackrel{a_{k}}{\rightarrow} y_{k}\right) \in \operatorname{pprem}(r)$. Then there is a proof of $\sigma\left(t_{k} \stackrel{a_{k}}{\rightarrow} y_{k}\right)$ from True $\left(\operatorname{Red}^{\alpha}(P)\right)$ that is less deep than the proof of $u \stackrel{a}{\rightarrow} u^{\prime}$. As $\sigma\left(t_{k} \stackrel{a_{k}}{\rightarrow} y_{k}\right) \in \rightarrow_{\text {True }\left(\operatorname{Red}^{\alpha}(P)\right)}$ and $\sigma\left(t_{k}\right) R_{P} \sigma^{\prime}\left(t_{k}\right)$, it follows by induction that $\exists w \in T(\Sigma): \sigma^{\prime}\left(t_{k}\right) \stackrel{a_{k}}{\rightarrow} w \in \rightarrow_{P}$ and $\sigma\left(y_{k}\right) R_{P} w$.

(ii) $\sigma\left(t_{k} \stackrel{a_{k}}{\rightarrow} y_{k}\right) \notin \operatorname{pprem}(r)$. Hence $\exists \beta<\alpha: \rightarrow_{\text {True }\left(\operatorname{Red}^{\beta}(P)\right)} \vDash \sigma\left(t_{k} \stackrel{a_{k}}{\rightarrow} y_{k}\right)$. As also $\sigma\left(t_{k}\right) R_{P} \sigma^{\prime}\left(t_{k}\right)$, it follows by induction that $\exists w \in T(\Sigma)$ : $\sigma^{\prime}\left(t_{k}\right) \stackrel{a_{k}}{\rightarrow} w \in \rightarrow_{P}$ and $\sigma\left(y_{k}\right) R_{P} w$.

In both cases, we take $\sigma^{\prime}\left(y_{k}\right)=w$. Note that the first three points of Claim 8.11 are satisfied, which finishes the first part of this proof. 
We are left to show that $\rightarrow_{P} \vDash \sigma^{\prime}\left(t_{l}\right)^{a_{l}} \not \rightarrow(l \in L)$. As $\sigma\left(t_{l}\right) R_{P} \sigma^{\prime}\left(t_{l}\right)$, it follows from point (1) of this lemma that $\sigma\left(t_{l}\right) \leftrightarrow_{P \Rightarrow P o s\left(\operatorname{Red}^{\alpha}(P)\right)} \sigma^{\prime}\left(t_{l}\right)$. In order to obtain a contradiction, assume that $\rightarrow_{P} \vDash \sigma^{\prime}\left(t_{l}\right) \stackrel{a_{l}}{\rightarrow} s^{\prime}$ for some $s^{\prime}$. Then $\rightarrow_{P o s\left(\operatorname{Red}^{\alpha}(P)\right)} \vDash \sigma\left(t_{l}\right) \stackrel{a_{l}}{\rightarrow}$ s for some $s$. So by Lemma 5.5 for all $\beta<$ $\alpha: \rightarrow_{P o s}\left(\operatorname{Red}^{\beta}(P)\right) \vDash \sigma\left(t_{l}\right) \stackrel{a_{l}}{\rightarrow} s$. This cannot be the case, as $\cap_{\beta<\alpha}$ $\rightarrow_{\operatorname{Pos}\left(\operatorname{Red}^{\beta}(P)\right)} \vDash \sigma\left(t_{l}\right)^{a_{l}} \rightarrow$.

From Claim 8.11, it follows that there is a substitution $\sigma^{\prime}$ such that $\rightarrow_{P} \vDash$ $\operatorname{prem}\left(\sigma^{\prime}\left(r^{\prime}\right)\right)$. Hence, $\rightarrow_{P} \vDash \operatorname{conc}\left(\sigma^{\prime}\left(r^{\prime}\right)\right)=v \stackrel{a}{\rightarrow} \sigma^{\prime}(t)$. Finally, as for all $x \in \operatorname{Var}\left(r^{\prime}\right): \sigma(x) R_{P} \sigma^{\prime}(x), u^{\prime}=\sigma(t) R_{P} \sigma^{\prime}(t)=v^{\prime}$.

LEMMA 8.12. Let $P$ be a pure TSS in ntyft-format that is positive after reduction. Then $R_{P}=\leftrightarrow_{P}$

Proof. As $P$ is positive after reduction for some ordinal $\alpha, \operatorname{Red}^{\alpha}(P)$ is positive. It follows using Corollary 5.8 that:

$$
\rightarrow_{P}=\rightarrow_{\operatorname{Red}^{\alpha}(P)}=\rightarrow_{\operatorname{Pos}_{\left(\operatorname{Red}^{\alpha}(P)\right)} .}
$$

Now, it follows using Lemma 8.9, the introduction of Definition 7.2 and the definition of $R_{P}$ that:

$$
R_{P} \subseteq \leftrightarrow_{P \Rightarrow P o s\left(\operatorname{Red}^{\alpha}(P)\right)}=\leftrightarrow_{P} \subseteq R_{P}
$$

Theorem 8.13 (CONGRUence TheOREM). Let $P$ be a TSS in ntyft/ntyxt-format that is positive after reduction. Then $\leftrightarrow_{P}$ is a congruence.

Proof. Assume $P=(\Sigma, A, R)$. According to Section 5.1 of Fokkink and van Glabbeek [1996] and Lemma 8.6 there is a pure TSS $P^{\prime}=\left(\Sigma, A, R^{\prime}\right)$ in $n$ tyft -format that is positive after reduction such that $\rightarrow_{P}=\rightarrow_{P^{\prime}}$. Hence, $\leftrightarrow_{P}=$ $\leftrightarrow_{P^{\prime}}$. By Lemma $8.12 \leftrightarrow_{P^{\prime}}=R_{P^{\prime}}$. As $R_{P^{\prime}}$ is a congruence with respect to $\Sigma$, $\leftrightarrow_{P}$ is also a congruence with respect to $\Sigma$.

The next example shows that the requirement in the Congruence Theorem 8.13 is that the TSS $P$ must be positive after reduction is really needed. We give a TSS in ntyft/ntyxt-format that has a unique stable transition relation but that is not positive after reduction and for which bisimulation is not a congruence.

Example 8.14. Let $P=(\Sigma, A, R)$ be a TSS where $\Sigma$ contains constants $c_{1}$ and $c_{2}$ and a unary function $f$. The actions in $A$ are $a, b_{1}, b_{2}$ and the rules are the following:

$$
\begin{aligned}
& \mathrm{E} 1: \quad c_{1} \stackrel{a}{\rightarrow} c_{1} \\
& \mathrm{E} 2: \quad c_{2} \stackrel{a}{\rightarrow} c_{2} \\
& \text { E3: } \frac{x \stackrel{a}{\rightarrow} y f(x) \stackrel{b_{1}}{\rightarrow} f\left(c_{1}\right)^{b_{2}} \nrightarrow}{f(x) \stackrel{b_{2}}{\rightarrow} c_{2}} \\
& \mathrm{E} 4: \frac{x \stackrel{a}{\rightarrow} y f(x) \stackrel{b_{2}}{\rightarrow} f\left(c_{2}\right)^{b_{1}} \rightarrow}{f} .
\end{aligned}
$$

Note that $P$ is pure and in $n t y f t$-format. $\operatorname{Red}^{1}(P)$ is a TSS with the following rules:

$$
\mathrm{E} 1^{\prime}: c_{1} \stackrel{a}{\rightarrow} c_{1} \quad \mathrm{E} 2^{\prime}: \quad c_{2} \stackrel{a}{\rightarrow} c_{2}
$$




$$
\begin{aligned}
& \mathrm{E}^{\prime}: \frac{f\left(c_{1}\right)^{b_{1}} \nrightarrow f\left(c_{1}\right)^{b_{2}} \nrightarrow}{f\left(c_{1}\right) \stackrel{b_{2}}{\rightarrow} c_{2}} \quad \mathrm{E}^{\prime \prime}: \frac{f\left(c_{2}\right)^{b_{1}} \nrightarrow}{f\left(c_{2}\right)^{\stackrel{b_{2}}{\rightarrow}} f\left(c_{1}\right)^{b_{2}} \nrightarrow} \\
& \mathrm{E} 4^{\prime}: \frac{f\left(c_{1}\right)^{b_{2}} \stackrel{\leftrightarrow}{\rightarrow} f\left(c_{2}\right)^{b_{1}} \nrightarrow}{f\left(c_{1}\right) \stackrel{b_{1}}{\rightarrow} c_{1}} \quad \mathrm{E} 4^{\prime \prime}: \frac{f\left(c_{2}\right)^{b_{2}} \nrightarrow f\left(c_{2}\right)^{b_{1}} \nrightarrow}{f\left(c_{2}\right) \stackrel{b_{1}}{\rightarrow} c_{1}} \text {. }
\end{aligned}
$$

Further reduction of $P$ is not possible. However, we observe that both in E3' and E4" the conclusion denies the second premise. Therefore, a transition relation that is stable for $P$ must deny the first premise of E3' and of E4", that is, it must contain $f\left(c_{1}\right) \stackrel{b_{1}}{\rightarrow} t_{1}$ and $f\left(c_{2}\right) \stackrel{b_{2}}{\rightarrow} t_{2}$ for some $t_{1}$ and $t_{2}$. The only candidates that might be provable are $f\left(c_{1}\right) \stackrel{b_{1}}{\rightarrow} c_{1}$ and $f\left(c_{2}\right) \stackrel{b_{2}}{\rightarrow} c_{2}$. Indeed they are provable from E3" and E4' (as blocking E3' and E4" implies $f\left(c_{1}\right)^{b_{2}} \nrightarrow$ and $f\left(c_{2}\right)^{b_{1}} \nrightarrow$ ), so $\left\{c_{1} \stackrel{a}{\rightarrow} c_{1}, c_{2} \stackrel{a}{\rightarrow} c_{2}, f\left(c_{1}\right) \stackrel{b_{1}}{\rightarrow} c_{1}, f\left(c_{2}\right) \stackrel{b_{2}}{\rightarrow} c_{2}\right\}$ is the unique transition relation that is stable for $P$. Now it is obvious that $c_{1} \leftrightarrow_{P} c_{2}$, but not $f\left(c_{1}\right) \leftrightarrow_{P} f\left(c_{2}\right)$, so $\leftrightarrow_{P}$ is not a congruence.

\section{Conservative extensions of TSSs}

It can be useful to enrich a given language with additional language constructs (as in our running example, where $\mathrm{BPA}_{\delta \epsilon \tau}$ is enriched with the priority and unless operator). For these new constructs, operational rules are devised which are added to the operational semantics of the basic language. In this section, we study how an operational semantics can be extended and especially how we can guarantee that transitions between terms in the basic language are not effected by the extension.

In this section, we assume that the operational semantics of the basic language is given by a TSS $P_{0}$. All extensions, that is, the added signature, label set and operational rules are given in a TSS $P_{1}$. The extension of $P_{0}$ with $P_{1}$ is written as $P_{0} \oplus P_{1}$ [Groote and Vaandrager 1992]. Due to the symmetric nature-we could as well extend $P_{1}$ with $P_{0}$-this is called the sum of $P_{0}$ and $P_{1}$.

Definition 9.1. Let $\Sigma_{i}=\left(F_{i}, \operatorname{rank}_{i}\right)(i=0,1)$ be two signatures such that for all $f \in F_{0} \cap F_{1}: \operatorname{rank}_{0}(f)=\operatorname{rank}_{1}(f)$. The sum of $\Sigma_{0}$ and $\Sigma_{1}$, notation $\Sigma_{0}$ $\oplus \Sigma_{1}$, is the signature:

$$
\Sigma_{0} \oplus \Sigma_{1}=\left(F_{0} \cup F_{1}, \lambda f \text {. if } f \in F_{0} \text {, then } \operatorname{rank}_{0}(f) \text {, else } \operatorname{rank}_{1}(f)\right) .
$$

Definition 9.2. Let $P_{i}=\left(\Sigma_{i}, A_{i}, R_{i}\right)(i=0,1)$ be two TSSs with $\Sigma_{0} \oplus \Sigma_{1}$ defined. The sum of $P_{0}$ and $P_{1}$, notation $P_{0} \oplus P_{1}$, is the TSS:

$$
P_{0} \oplus P_{1}=\left(\Sigma_{0} \oplus \Sigma_{1}, A_{0} \cup A_{1}, R_{0} \cup R_{1}\right) \text {. }
$$

If $P_{0}$ is extended with $P_{1}$ such that 'the properties' of $P_{0}$ are maintained, $P_{0} \oplus P_{1}$ is said to be a conservative extension of $P_{0}$. By 'properties' of $P_{0}$ we mean transitions that can be performed by terms over the signature of $P_{0}$. To be more precise: 
Definition 9.3. Let $P_{i}=\left(\Sigma_{i}, A_{i}, R_{i}\right)(i=0,1)$ be two TSSs such that $P_{0}$ has associated transition relation $\rightarrow P_{0}$. Let $P_{0} \oplus P_{1}$ with associated transition relation $\rightarrow{ }_{P_{0} \oplus P_{1}}$ be defined. We say that $P_{0} \oplus P_{1}$ is a conservative extension of $P_{0}$ and that $P_{1}$ can be added conservatively to $P_{0}$ if

$$
\rightarrow_{P_{0} \oplus P_{1}} \cap\left(T\left(\Sigma_{0}\right) \times\left(A_{0} \cup A_{1}\right) \times T\left(\Sigma_{0} \oplus \Sigma_{1}\right)\right)=\rightarrow_{P_{0}} .
$$

An alternative formulation of the same definition has been given in Groote and Vaandrager [1992]. Adapting that definition to our terminology, it says that $P=P_{0} \oplus P_{1}=(\Sigma, A, R)$ with associated transition relation $\rightarrow_{P}$ is a conservative extension of $P_{0}=\left(\Sigma_{0}, A_{0}, R_{0}\right)$ if for all $t \in T\left(\Sigma_{0}\right), a \in A$ and $t^{\prime} \in T(\Sigma):$

$$
\rightarrow_{P} \vDash t \stackrel{a}{\rightarrow} t^{\prime} \Leftrightarrow \rightarrow_{P_{0}} \vDash t \stackrel{a}{\rightarrow} t^{\prime},
$$

where $\rightarrow_{P_{0}}$ is associated with $P_{0}$.

We now head for a theorem that gives conditions under which $P_{1}$ can be added conservatively to $P_{0}$. It turns out that this is the case if $P_{0}$ is pure and each rule in $P_{1}$ contains a function name in the source of its conclusion that does not appear in the signature of $P_{0}$. This theorem considerably extends the results in Groote [1993] in which a comparable theorem was proved for TSSs in $n t y f t / n t y x t$-format. If our result is restricted to this format, both results coincide, except that here, we deal with TSSs that are positive after reduction while in Groote [1993] only stratified TSSs were considered.

LEMMA 9.4. Let $\Sigma_{0}=\left(F_{0}\right.$, rank $\left.k_{0}\right)$ be a signature. Let $P_{0}=\left(\Sigma_{0}, A_{0}, R_{0}\right)$ be a pure TSS and let $P_{1}=\left(\Sigma_{1}, A_{1}, R_{1}\right)$ be a TSS such that $P_{0} \oplus P_{1}$ is defined and for each rule $r \in R_{1}$ : source $(\operatorname{conc}(r)) \notin \mathbb{T}\left(\Sigma_{0}\right)$. Then, for each ordinal $\alpha$ :

$$
\begin{aligned}
& \rightarrow_{\operatorname{Pos}\left(\operatorname{Red}^{\alpha}\left(P_{0} \oplus P_{1}\right)\right)} \cap\left(T\left(\Sigma_{0}\right) \times\left(A_{0} \cup A_{1}\right) \times T\left(\Sigma_{0} \oplus \Sigma_{1}\right)\right)=\rightarrow_{\operatorname{Pos}\left(\operatorname{Red}^{\alpha}\left(P_{0}\right)\right)} \\
& \rightarrow_{\operatorname{True}\left(\operatorname{Red} \alpha\left(P_{0} \oplus P_{1}\right)\right)} \cap\left(T\left(\Sigma_{0}\right) \times\left(A_{0} \cup A_{1}\right) \times T\left(\Sigma_{0} \oplus \Sigma_{1}\right)\right)=\rightarrow_{\operatorname{True}\left(\operatorname{Red} \alpha\left(P_{0}\right)\right)}
\end{aligned}
$$

Proof. We prove clauses (1) and (2) by simultaneous induction on $\alpha$.

(1) $\subseteq$ For this case it is sufficient to show the following:

$$
\begin{gathered}
\operatorname{Pos}\left(\operatorname{Red}^{\alpha}\left(P_{0} \oplus P_{1}\right)\right) \vdash t \stackrel{a}{\rightarrow} t^{\prime} \text { and } t \in T\left(\Sigma_{0}\right) \text { implies } \\
\operatorname{Pos}\left(\operatorname{Red}^{\alpha}\left(P_{0}\right)\right) \vdash t \stackrel{a}{\rightarrow} t^{\prime}, a \in A_{0} \text { and } t^{\prime} \in T\left(\Sigma_{0}\right) .
\end{gathered}
$$

So assume that $\operatorname{Pos}\left(\operatorname{Red}^{\alpha}\left(P_{0} \oplus P_{1}\right)\right) \vdash t \stackrel{a}{\rightarrow} t^{\prime}$ and $t \in T\left(\Sigma_{0}\right)$. We use induction on the depth of this proof. Let the last rule of this proof be $r \in$ $\operatorname{Pos}\left(\operatorname{Red}^{\alpha}\left(R_{0} \oplus R_{1}\right)\right)$. Then $\operatorname{conc}(r)=t \stackrel{a}{\rightarrow} t^{\prime}$. Hence, as $t \in T\left(\Sigma_{0}\right)$ and all rules in $R_{1}$ contain a function name $f \notin \Sigma_{0}$ in the source of their conclusions, $r$ is derived from a rule $\sigma\left(r^{\prime}\right)$ with $r^{\prime} \in R_{0}$. So $a \in A_{0}$.

ClaIM 9.5. For all $x \in \operatorname{Var}\left(r^{\prime}\right): \sigma(x) \in T\left(\Sigma_{0}\right)$.

Proof of Claim 9.5. As $r^{\prime}$ is pure, it is well-founded, so pprem $\left(r^{\prime}\right)$ has a variable dependency graph VDG. We prove the claim by induction on $n_{V D G}(x)$. Consider some $x$ with $n_{V D G}(x)=\gamma$ and assume the claim holds for all $x^{\prime}$ such that $n_{V D G}\left(x^{\prime}\right)<\gamma$. As $r^{\prime}$ does not contain free variables, one of the following two cases must hold: 
(1) $x \in \operatorname{Var}\left(\operatorname{source}\left(\operatorname{conc}\left(r^{\prime}\right)\right)\right.$. As $\sigma\left(\operatorname{source}\left(\operatorname{conc}\left(r^{\prime}\right)\right) \in T\left(\Sigma_{0}\right), \sigma(x)\right.$ $\in T\left(\Sigma_{0}\right)$.

(2) $x \in \operatorname{Var}\left(u^{\prime}\right)$ and $u \stackrel{a}{\rightarrow} u^{\prime} \in \operatorname{pprem}\left(r^{\prime}\right)$. For all $x^{\prime} \in \operatorname{Var}(u): n_{V D G}\left(x^{\prime}\right)$ $<n_{V D G}(x)$ and therefore $\sigma\left(x^{\prime}\right) \in T\left(\Sigma_{0}\right)$. Hence, $\sigma(u) \in T\left(\Sigma_{0}\right)$. Distinguish the following two cases:

(a)

$$
\bigcup_{\beta<\alpha} \rightarrow_{\operatorname{True}\left(\operatorname{Red}\left(P_{0} \oplus P_{1}\right)\right)} \vDash \sigma(u) \stackrel{a}{\rightarrow} \sigma\left(u^{\prime}\right) .
$$

Then by (2),

$$
\bigcup_{\beta<\alpha} \rightarrow_{\text {True }\left(\operatorname{Red}\left(P_{0}\right)\right)} \vDash \sigma(u) \stackrel{a}{\rightarrow} \sigma\left(u^{\prime}\right)
$$

and this means that $\sigma\left(u^{\prime}\right) \in T\left(\Sigma_{0}\right)$. Therefore, as $x \in \operatorname{Var}\left(u^{\prime}\right)$, $\sigma(x) \in T\left(\Sigma_{0}\right)$.

(b)

$$
\bigcup_{\beta<\alpha} \rightarrow_{\text {True }\left(\operatorname{Red}^{\beta}\left(P_{0} \oplus P_{1}\right)\right)} \not \neq \sigma(u) \stackrel{a}{\rightarrow} \sigma\left(u^{\prime}\right) .
$$

Then, $\sigma(u) \stackrel{a}{\rightarrow} \sigma\left(u^{\prime}\right) \in \operatorname{pprem}(r)$ and therefore,

$$
\operatorname{Pos}\left(\operatorname{Red}^{\alpha}\left(P_{0} \oplus P_{1}\right)\right) \vdash \sigma(u) \stackrel{a}{\rightarrow} \sigma\left(u^{\prime}\right) .
$$

By induction (on the proof tree), it follows that:

$$
\operatorname{Pos}\left(\operatorname{Red}^{\alpha}\left(P_{0}\right)\right) \vdash \sigma(u) \stackrel{a}{\rightarrow} \sigma\left(u^{\prime}\right)
$$

and $\sigma\left(u^{\prime}\right) \in T\left(\Sigma_{0}\right)$. Hence, $\sigma(x) \in T\left(\Sigma_{0}\right)$.

As $r$ is derived from reducing $\sigma\left(r^{\prime}\right)$, we have the following:

$$
\begin{aligned}
& \cap_{\beta<\alpha} \rightarrow_{\operatorname{Pos}\left(\operatorname{Red} \beta\left(P_{0} \oplus P_{1}\right)\right)} \vDash \operatorname{pprem}\left(\sigma\left(r^{\prime}\right)\right), \\
& \cup_{\beta<\alpha} \rightarrow_{\text {True }\left(\operatorname{Red}^{\beta}\left(P_{0} \oplus P_{1}\right)\right)} \vDash \operatorname{nprem}\left(\sigma\left(r^{\prime}\right)\right), \\
& \cup_{\beta<\alpha} \rightarrow_{\text {True }\left(\operatorname{Red}^{\beta}\left(P_{0} \oplus P_{1}\right)\right)} \vDash \operatorname{pprem}\left(\sigma\left(r^{\prime}\right)\right)-\operatorname{prem}(r) .
\end{aligned}
$$

As by Claim $9.5 \sigma: \operatorname{Var}\left(r^{\prime}\right) \rightarrow T\left(\Sigma_{0}\right)$, it follows using the outermost induction hypothesis that:

$$
\begin{aligned}
& \cap_{\beta<\alpha} \rightarrow_{\text {Pos }\left(\operatorname{Red}\left(P_{0}\right)\right)} \vDash \operatorname{pprem}\left(\sigma\left(r^{\prime}\right)\right), \\
& \cup_{\beta<\alpha} \rightarrow_{\text {True }\left(\operatorname{Red} \beta\left(P_{0}\right)\right)} \vDash \operatorname{nprem}\left(\sigma\left(r^{\prime}\right)\right), \\
& \cup_{\beta<\alpha} \rightarrow_{\text {True }\left(\operatorname{Red} d^{\beta}\left(P_{0}\right)\right)} \vDash \operatorname{pprem}\left(\sigma\left(r^{\prime}\right)\right)-\operatorname{prem}(r) .
\end{aligned}
$$

Or in other words $r \in \operatorname{Pos}\left(\operatorname{Red}^{\alpha}\left(R_{0}\right)\right)$. By induction on the proof tree and Claim 9.5, it follows that $\operatorname{Pos}\left(\operatorname{Red}^{\alpha}\left(P_{0}\right)\right) \vdash \operatorname{prem}(r)$ and therefore $\operatorname{Pos}\left(\operatorname{Red}^{\alpha}\left(P_{0}\right)\right) \vdash t \stackrel{a}{\rightarrow} t^{\prime}=\sigma\left(\operatorname{conc}\left(r^{\prime}\right)\right) \in \operatorname{Tr}\left(\Sigma_{0}, A_{0}\right)$.

(1) $\supseteq$ For this case it is sufficient to prove (using induction on the proof tree for $\left.\operatorname{Pos}\left(\operatorname{Red}^{\alpha}\left(P_{0}\right)\right) \vdash t \stackrel{a}{\rightarrow} t^{\prime}\right)$ that:

$$
\operatorname{Pos}\left(\operatorname{Red}^{\alpha}\left(P_{0}\right)\right) \vdash t \stackrel{a}{\rightarrow} t^{\prime} \Rightarrow \operatorname{Pos}\left(\operatorname{Red}^{\alpha}\left(P_{0} \oplus P_{1}\right)\right) \vdash t \stackrel{a}{\rightarrow} t^{\prime} .
$$


So assume $r \in \operatorname{Pos}\left(\operatorname{Red}^{\alpha}\left(R_{0}\right)\right)$ is the last rule used in the proof for $t \stackrel{a}{\rightarrow} t^{\prime}$. Hence, there is a rule $r^{\prime} \in R_{0}$ and a substitution $\sigma: \operatorname{Var}\left(r^{\prime}\right) \rightarrow$ $T\left(\Sigma_{0}\right)$ with $\operatorname{conc}\left(\sigma\left(r^{\prime}\right)\right)=\operatorname{conc}(r), \operatorname{prem}(r) \subseteq \operatorname{pprem}\left(\sigma\left(r^{\prime}\right)\right)$. Moreover:

$$
\begin{aligned}
& \cap_{\beta<\alpha} \rightarrow{ }_{P o s\left(\operatorname{Red} d^{\beta}\left(P_{0}\right)\right)} \vDash \operatorname{pprem}\left(\sigma\left(r^{\prime}\right)\right), \\
& \cup_{\beta<\alpha} \rightarrow_{\operatorname{True}\left(\operatorname{Red}^{\beta}\left(P_{0}\right)\right)} \vDash n \operatorname{prem}\left(\sigma\left(r^{\prime}\right)\right), \\
& \cup_{\beta<\alpha} \rightarrow{ }_{\operatorname{True}\left(\operatorname{Red}^{\beta}\left(P_{0}\right)\right)} \vDash \operatorname{pprem}\left(\sigma\left(r^{\prime}\right)\right)-\operatorname{prem}(r) .
\end{aligned}
$$

As for each premise $\psi \in \operatorname{prem}\left(\sigma\left(r^{\prime}\right)\right)$, source $(\psi) \in T\left(\Sigma_{0}\right)$, we have by induction:

$$
\begin{aligned}
& \cap_{\beta<\alpha} \rightarrow{ }_{P o s\left(\operatorname{Red}^{\beta}\left(P_{0} \oplus P_{1}\right)\right)} \vDash \operatorname{pprem}\left(\sigma\left(r^{\prime}\right)\right), \\
& \cup_{\beta<\alpha} \rightarrow{ }_{\operatorname{True}\left(\operatorname{Red}^{\beta}\left(P_{0} \oplus P_{1}\right)\right)} \vDash \operatorname{nprem}\left(\sigma\left(r^{\prime}\right)\right), \\
& \cup_{\beta<\alpha} \rightarrow{ }_{\operatorname{True}\left(\operatorname{Red}^{\beta}\left(P_{0} \oplus P_{1}\right)\right)} \vDash \operatorname{pprem}\left(\sigma\left(r^{\prime}\right)\right)-\operatorname{prem}(r) .
\end{aligned}
$$

Hence, $r \in \operatorname{Pos}\left(\operatorname{Red}^{\alpha}\left(R_{0} \oplus R_{1}\right)\right)$. As $\operatorname{Pos}\left(\operatorname{Red}^{\alpha}\left(P_{0}\right)\right) \quad \vdash \quad t \stackrel{a}{\rightarrow} t^{\prime}$, $\operatorname{Pos}\left(\operatorname{Red}^{\alpha}\left(P_{0}\right)\right) \vdash \psi$ for each $\psi \in \operatorname{prem}(r)$. By induction $\operatorname{Pos}\left(\operatorname{Red}^{\alpha}\right.$ $\left.\left(P_{0} \oplus P_{1}\right)\right) \vdash \psi$ and hence, $\operatorname{Pos}\left(\operatorname{Red}^{\alpha}\left(P_{0} \oplus P_{1}\right)\right) \vdash t \stackrel{a}{\rightarrow} t^{\prime}$.

(2) This case can be shown in the same way as (1).

Theorem 9.6 (Conservativity). Let $\Sigma_{0}=\left(F_{0}\right.$, rank $\left.k_{0}\right)$ be a signature. Let $P_{0}=\left(\Sigma_{0}, A_{0}, R_{0}\right)$ be a pure TSS and let $P_{1}=\left(\Sigma_{1}, A_{1}, R_{1}\right)$ be a TSS such that each rule $r \in R_{1}$ contains at least one function name $f \notin F_{0}$ in the source of its conclusion. Furthermore, assume that $P_{0} \oplus P_{1}$ exists and is positive after reduction. Then $P_{0} \oplus P_{1}$ is a conservative extension of $P_{0}$.

Proof. As $P_{0} \oplus P_{1}$ is positive after reduction, there is some ordinal $\alpha$ such that $\operatorname{Red}^{\alpha}\left(P_{0} \oplus P_{1}\right)$ is a positive TSS. Hence, $P_{0} \oplus P_{1}$ has an associated transition relation $\rightarrow_{P_{0} \oplus P_{1}}$. Let $A=A_{0} \cup A_{1}$ and $\Sigma=\Sigma_{0} \oplus \Sigma_{1}$. By Lemma 9.4, we have:

$$
\begin{aligned}
& \rightarrow_{\operatorname{Pos}\left(\operatorname{Red}^{\alpha}\left(P_{0}\right)\right)}= \\
& \rightarrow_{P o s\left(\operatorname{Red}^{\alpha}\left(P_{0} \oplus P_{1}\right)\right)} \cap\left(T\left(\Sigma_{0}\right) \times A \times T(\Sigma)\right)= \\
& \rightarrow_{\text {True }\left(\operatorname{Red}^{\alpha}\left(P_{0} \oplus P_{1}\right)\right)} \cap\left(T\left(\Sigma_{0}\right) \times A \times T(\Sigma)\right)= \\
& \rightarrow_{\text {True }\left(\operatorname{Red}^{\alpha}\left(P_{0}\right)\right) .}
\end{aligned}
$$

Hence, by Remark 5.2, $\operatorname{Red}^{\alpha+1}\left(P_{0}\right)$ is a positive TSS. Therefore, $P_{0}$ also has an associated transition relation $\rightarrow_{P_{0}}$. Moreover, using Corollary 5.8 and Lemma 9.4, we have:

$$
\begin{aligned}
& \rightarrow_{P_{0}}= \\
& \rightarrow_{\text {True }\left(\operatorname{Red}^{\alpha+1}\left(P_{0}\right)\right)}= \\
& \rightarrow_{\text {True }\left(\operatorname{Red}^{\alpha+1}\left(P_{0} \oplus P_{1}\right)\right)} \cap\left(T\left(\Sigma_{0}\right) \times A \times T(\Sigma)\right)=
\end{aligned}
$$


TABLE II. The Axiom Set BPA ${ }_{\delta \epsilon \tau}^{\theta}\left(a, b \in A c t_{\tau}\right.$ AND $\left.c \in A c t\right)$.

\begin{tabular}{|ll|ll|}
\hline$x+(y+z)=(x+y)+z$ & $\mathrm{~A} 1$ & $a \tau=a$ & $\mathrm{~T} 1$ \\
$x+y=y+x$ & $\mathrm{~A} 2$ & $\tau x+x=\tau x$ & $\mathrm{~T} 2$ \\
$x+x=x$ & $\mathrm{~A} 3$ & $a(\tau x+y)=a(\tau x+y)+a x$ & $\mathrm{~T} 3$ \\
$(x+y) z=x z+y z$ & $\mathrm{~A} 4$ & & \\
$(x y) z=x(y z)$ & $\mathrm{A} 5$ & & $\mathrm{THE}$ \\
$x+\delta=x$ & $\mathrm{~A} 6$ & $\theta(\epsilon)=\epsilon$ & $\mathrm{THD}$ \\
$\delta x=\delta$ & $\mathrm{A} 7$ & $\theta(\delta)=\delta$ & $\mathrm{TH} 1$ \\
$\epsilon x=x$ & $\mathrm{~A} 8$ & $\theta(a x)=a \theta(x)$ & $\mathrm{TH} 2$ \\
$x \epsilon=x$ & $\mathrm{~A} 9$ & $\theta(x+y)=\theta(x) \triangleleft y+\theta(y) \triangleleft x$ & $\mathrm{TIE}$ \\
& $\mathrm{PE} 1$ & $\tau_{I}(\epsilon)=\epsilon$ & $\mathrm{TID}$ \\
$\epsilon \triangleleft x=\epsilon$ & $\mathrm{PE} 2$ & $\tau_{I}(\delta)=\delta$ & $\mathrm{TI} 1$ \\
$x \triangleleft \epsilon=x$ & $\mathrm{PD} 1$ & $\tau_{I}(a)=a$ if $a \notin I$ & $\mathrm{TI} 2$ \\
$\delta \triangleleft x=\delta$ & $\mathrm{PD} 2$ & $\tau_{I}(a)=\tau$ if $a \in I$ & $\mathrm{TI} 3$ \\
$x \triangleleft \delta=x$ & $\mathrm{P} 1$ & $\tau_{I}(x+y)=\tau_{I}(x)+\tau_{I}(y)$ & $\mathrm{TI} 4$ \\
$a x \triangleleft b y=\delta$ if $(a<b)$ & $\mathrm{P} 2$ & $\tau_{I}(x y)=\tau_{I}(x) \tau_{I}(y)$ & \\
$a x \triangleleft c y=a x$ if $\neg(a<c)$ & $\mathrm{P} 3$ & & \\
$a x \triangleleft \tau y=a x \triangleleft y$ if $\neg(a<\tau)$ & $\mathrm{P} 4$ & & \\
$x \triangleleft(y+z)=(x \triangleleft y) \triangleleft z$ & $\mathrm{P} 5$ & & \\
$(x+y) \triangleleft z=x \triangleleft z+y \triangleleft z$ & $\mathrm{P}$ & \\
\end{tabular}

Remark 9.7. From the alternative definition of conservativity it is immediately obvious that if $P_{0} \oplus P_{1}$ is a conservative extension of $P_{0}=\left(\Sigma_{0}, A_{0}, R_{0}\right)$ then for all $t, u \in T\left(\Sigma_{0}\right): t \leftrightarrow_{P_{0}} u \Leftrightarrow t \leftrightarrow_{P_{0} \oplus P_{1}} u$.

Example 9.8. We can apply the Conservativity Theorem to show that the priority operator and the unless operator form a conservative extension of $\mathrm{BPA}_{\delta \epsilon \tau}$. We can also conservatively add the parallel operator which is characterized by the following rules:

$$
10.1 \frac{x \stackrel{a}{\rightarrow} x^{\prime}}{x\left\|y \stackrel{a}{\rightarrow} x^{\prime}\right\| y} \quad 10.2 \quad \frac{y \stackrel{a}{\rightarrow} y^{\prime}}{x\|y \stackrel{a}{\rightarrow} x\| y^{\prime}}
$$

to $\mathrm{BPA}_{\delta \epsilon \tau}$ with priorities. In fact in almost all cases the addition of new operators to an existing TSS turns out to be conservative.

\section{An axiomatization of priorities with abstraction}

This last section is devoted to our running example. We consider an instance $P_{\theta}=\left(\Sigma_{\theta}, A_{\theta}, R_{\theta}\right)$ of $\mathrm{BPA}_{\delta \epsilon \tau}$ with priorities such that for all $\left(X \Leftarrow t_{X}\right) \in$ $E: \tau_{I}(\cdot)$ does not occur in $t_{X}$ and for all $a \in A c t$ it does not hold that $\tau<a$. By Theorem $6.6 P_{\theta}$ has an associated transition relation $\rightarrow_{P_{\theta}}$.

In Table II, we list the axiom set $\mathrm{BPA}_{\delta \epsilon \tau}^{\theta}$ for strong bisimulation equivalence induced by $P_{\theta}$. This axiom system consists of a straightforward assembly of 
existing axioms [Baeten et al. 1986; Milner 1980], adding only the axiom P3 showing the interaction between $\triangleleft$ and $\tau$. Nevertheless, as far as we know, this straightforward compilation has not been justified in bisimulation semantics. Only in Vaandrager [1990] $\tau$ and $\theta$ have been combined using an isomorphic embedding.

This section is added to show how an axiom system can be proved sound and complete with respect to an operational semantics, even if this semantics is defined using negative premises. We give all essential lemmas and theorems but only some insightful parts of the proofs. Most proofs apply induction on proof trees (standard for positive TSSs) of the 'stripped' TSS. This leads to a more general observation: induction on proof trees derived from a 'stripped' TSS is a powerful proof tool for TSSs with negative premises.

Definition 10.1. Let $\Sigma=(F$, rank $)$ be a signature and let $E q$ be a set of axioms over $\Sigma$. Let $R_{E q} \subseteq T(\Sigma) \times T(\Sigma)$ be the smallest congruence relation satisfying that $t R_{E q} u$ if $t=u$ is a ground instance of an axiom in $E q$. For terms $t, u \in T(\Sigma)$, we say that $E q$ proves $t=u$, notation $E q \vdash t=u$, if $t R_{E q} u$.

The following lemma says how behavior of a complex term can be explained in terms of necessary behavior of its components. This lemma is first used in Vaandrager [1990] to prove the soundness of the axioms. Due to rules R9.2 and $\mathrm{R} 9.3$, the proof of this lemma is lengthy.

Lemma 10.2 (Structuring Lemma). Let $t, u, v \in T\left(\Sigma_{\theta}\right)$ and $a \in A_{\theta}$. If $t+u \stackrel{a}{\rightarrow} v$, then one of the following must hold:

(1) $t \stackrel{a}{\rightarrow} v$,

(2) $u \stackrel{a}{\rightarrow} v$.

If $t \cdot u \stackrel{a}{\rightarrow} v$, then one of the following must hold:

(1) $t \stackrel{a}{\rightarrow} t^{\prime}, v \equiv t^{\prime} \cdot u$ and $a \not \equiv \vee$ for some $t^{\prime} \in T\left(\Sigma_{\theta}\right)$,

(2) $t \stackrel{\vee}{\rightarrow} t^{\prime}$ and $u \stackrel{a}{\rightarrow} v$ for some $t^{\prime} \in T\left(\Sigma_{\theta}\right)$,

(3) $t \stackrel{a}{\rightarrow} t^{\prime}, t^{\prime} \stackrel{\vee}{\rightarrow} t^{\prime \prime}, u \stackrel{\tau}{\rightarrow} v$ and $a \not \equiv \vee$ for some $t^{\prime}, t^{\prime \prime} \in T\left(\Sigma_{\theta}\right)$.

If $\theta(t) \stackrel{a}{\rightarrow} u$, then one of the following must hold:

(1) $t \stackrel{a}{\rightarrow} t^{\prime}, u \equiv \theta\left(t^{\prime}\right), a \not \equiv \vee$ and $\forall b>a t^{b} \nrightarrow$ for some $t^{\prime} \in T\left(\Sigma_{\theta}\right)$,

(2) $t \stackrel{\tau}{\rightarrow} t^{\prime}, t^{\prime} \stackrel{a}{\rightarrow} t^{\prime \prime}, u \equiv \theta\left(t^{\prime \prime}\right), a \neq \equiv$ and $\forall b>a t^{\prime} \stackrel{b}{\rightarrow}$ for some $t^{\prime}, t^{\prime \prime} \in T\left(\Sigma_{\theta}\right)$,

(3) $t \stackrel{\vee}{\rightarrow} t^{\prime}, u \equiv \theta\left(t^{\prime}\right)$ and $a \not \equiv \vee$ for some $t^{\prime} \in T\left(\Sigma_{\theta}\right)$.

If $t \triangleleft u \stackrel{a}{\rightarrow} v$, then one of the following must hold:

(1) $t \stackrel{a}{\rightarrow} v, a \neq \vee$ and $\forall b>a u \stackrel{b}{\rightarrow}$,

(2) $t \stackrel{\tau}{\rightarrow} t^{\prime}, t^{\prime} \stackrel{a}{\rightarrow} v$ and $a \not \equiv \vee$ for some $t^{\prime} \in T\left(\Sigma_{\theta}\right)$,

(3) $t \stackrel{\vee}{\rightarrow} v$ and $a \equiv V$.

If $\tau_{I}(t) \stackrel{a}{\rightarrow} u$, then one of the following must hold:

(1) $t \stackrel{a_{1}}{\rightarrow} t_{1} \stackrel{a_{2}}{\rightarrow} \cdots \stackrel{a_{n}}{\rightarrow} t_{n} \stackrel{a}{\rightarrow} t_{n+1} \stackrel{a_{n+2}}{\rightarrow} \cdots \stackrel{a_{m}}{\rightarrow} t_{m}, a \notin I$ and $u \equiv \tau_{I}\left(t_{m}\right)$ for some $a_{1}, \ldots$, $a_{n}, a_{n+2}, \ldots, a_{m} \in I, t_{1}, \ldots, t_{m} \in T\left(\Sigma_{\theta}\right), n \geq 0$ and $m \geq 1$. 
(2) $t \stackrel{a_{1}}{\rightarrow} t_{1} \stackrel{a_{2}}{\rightarrow} \ldots \stackrel{a_{n}}{\rightarrow} t_{n}, a \equiv \tau$ and $u \equiv \tau_{I}$ for some $a_{1}, \ldots, a_{n} \in I, t_{1}, \ldots, t_{n} \in$ $T\left(\Sigma_{\theta}\right)$ and $n \geq 1$.

Proof. As an illustration, we give the proof for $\theta(t) \stackrel{a}{\rightarrow} u$ in case $a \not \equiv \vee V$. All other proofs can be given in the same way.

If $\theta(t) \stackrel{a}{\rightarrow} u$, then this is equivalent to saying that $\operatorname{Strip}\left(P_{\theta}, \rightarrow_{P_{\theta}}\right) \vdash \theta(t) \stackrel{a}{\rightarrow} u$. We show with induction on the proof tree that $\operatorname{Strip}\left(P_{\theta}, \rightarrow_{P_{\theta}}\right) \vdash \theta(t) \stackrel{a}{\rightarrow} u$ implies that one of the following holds:

(1) $t \stackrel{a}{\rightarrow} t^{\prime}, u \equiv \theta\left(t^{\prime}\right)$ and $\forall b>a t^{b} \nrightarrow$ for some $t^{\prime} \in T\left(\Sigma_{\theta}\right)$,

(2) $t \stackrel{\tau}{\rightarrow} t^{\prime}, t^{\prime} \stackrel{a}{\rightarrow} t^{\prime \prime}, u \equiv \theta\left(t^{\prime \prime}\right)$ and $\forall b>a t^{\prime} \stackrel{b}{\rightarrow}$ for some $t^{\prime}, t^{\prime \prime} \in T\left(\Sigma_{\theta}\right)$.

Suppose $\operatorname{Strip}\left(P_{\theta}, \rightarrow_{P_{\theta}}\right) \vdash \theta(t) \stackrel{a}{\rightarrow} u$. The last rule that is used in this proof must either be R9.2, R9.3 or a stripped version of R5.1. Suppose a simplified version of rule R5.1 has been used. In this case the premises of R5.1, $t \stackrel{a}{\rightarrow} t^{\prime}$ and $\forall b>a t^{b} \nrightarrow$, hold in $\rightarrow_{P_{\theta}}$. Furthermore, $u \equiv \theta\left(t^{\prime}\right)$. So case (1) of $\theta$ in the Structuring Lemma must hold.

If rule R9.2 has been used, we know that $\operatorname{Strip}\left(P_{\theta}, \rightarrow_{P_{\theta}}\right) \vdash \theta(t) \stackrel{\tau}{\rightarrow} u^{\prime}$ and $\operatorname{Strip}\left(P_{\theta}, \rightarrow_{P_{\theta}}\right) \vdash u^{\prime} \stackrel{a}{\rightarrow} u$. By induction one of the following four cases must hold:

(1) $t \stackrel{\tau}{\rightarrow} t^{\prime}, t^{\prime} \stackrel{a}{\rightarrow} t^{\prime \prime}, \forall b>a t^{\prime} \stackrel{b}{\rightarrow}$ and $u \equiv \theta\left(t^{\prime \prime}\right)$,

(2) $t \stackrel{\tau}{\rightarrow} t^{\prime}, t^{\prime} \stackrel{\tau}{\rightarrow} t^{\prime \prime}, t^{\prime \prime} \stackrel{a}{\rightarrow} t^{\prime \prime \prime}, \forall b>a t^{\prime \prime} \stackrel{b}{\rightarrow}$ and $u \equiv \theta\left(t^{\prime \prime \prime}\right)$,

(3) $t \stackrel{\tau}{\rightarrow} t^{\prime}, t^{\prime} \stackrel{\tau}{\rightarrow} t^{\prime \prime}, t^{\prime \prime} \stackrel{a}{\rightarrow} t^{\prime \prime \prime}, \forall b>a t^{\prime \prime} \stackrel{b}{\rightarrow}$ and $u \equiv \theta\left(t^{\prime \prime \prime}\right)$,

(4) $t \stackrel{\tau}{\rightarrow} t^{\prime}, t^{\prime} \stackrel{\tau}{\rightarrow} t^{\prime \prime}, t^{\prime \prime} \stackrel{\tau}{\rightarrow} t^{\prime \prime \prime}, t^{\prime \prime \prime} \stackrel{a}{\rightarrow} t^{\prime \prime \prime \prime}, \forall b>a t^{\prime \prime \prime} \stackrel{b}{\rightarrow}$ and $u \equiv \theta\left(t^{\prime \prime \prime \prime}\right)$.

In all cases, it must hold that for some $v$ and $v^{\prime}$ :

$$
t \stackrel{\tau}{\rightarrow} v, v \stackrel{a}{\rightarrow} v^{\prime}, \forall b>a v \stackrel{b}{\rightarrow} \text { and } u \equiv \theta\left(v^{\prime}\right) .
$$

Suppose rule $\mathrm{R} 9.3$ has been used as last step in the proof. As the premises of R9.3 are derivable, we have:

$$
\operatorname{Strip}\left(P_{\theta}, \rightarrow_{P_{\theta}}\right) \vdash \theta(t) \stackrel{a}{\rightarrow} u^{\prime}, \operatorname{Strip}\left(P_{\theta}, \rightarrow_{P_{\theta}}\right) \vdash u^{\prime} \stackrel{\tau}{\rightarrow} u .
$$

By induction one of the following four cases must hold:

(1) $t \stackrel{a}{\rightarrow} t^{\prime}, t^{\prime} \stackrel{\tau}{\rightarrow} t^{\prime \prime}, \forall b>a t \stackrel{b}{\rightarrow}$ and $u \equiv \theta\left(t^{\prime \prime}\right)$,

(2) $t \stackrel{a}{\rightarrow} t^{\prime}, t^{\prime} \stackrel{\tau}{\rightarrow} t^{\prime \prime}, t^{\prime \prime} \stackrel{\tau}{\rightarrow} t^{\prime \prime \prime}, \forall b>a t^{b} \nrightarrow$ and $u \equiv \theta\left(t^{\prime \prime \prime}\right)$,

(3) $t \stackrel{\tau}{\rightarrow} t^{\prime}, t^{\prime} \stackrel{a}{\rightarrow} t^{\prime \prime}, t^{\prime \prime} \stackrel{\tau}{\rightarrow} t^{\prime \prime \prime}, \forall b>a t^{\prime b} \nrightarrow$ and $u \equiv \theta\left(t^{\prime \prime \prime}\right)$,

(4) $t \stackrel{\tau}{\rightarrow} t^{\prime}, t^{\prime} \stackrel{a}{\rightarrow} t^{\prime \prime}, t^{\prime \prime} \stackrel{\tau}{\rightarrow} t^{\prime \prime \prime}, t^{\prime \prime \prime} \stackrel{\tau}{\rightarrow} t^{\prime \prime \prime \prime}, \forall b>a t^{\prime b} \nrightarrow$ and $u \equiv \theta\left(t^{\prime \prime \prime \prime}\right)$.

From cases (1) or (2), it follows that (for appropriate $v \in T\left(\Sigma_{\theta}\right)$ ):

$$
t \stackrel{a}{\rightarrow} v, \forall b>a t^{b} \nrightarrow \text { and } u \equiv \theta(v)
$$

which is case (1) for $\theta$ in the Structuring Lemma. From cases (3) or (4), it follows that (for appropriate $v, v^{\prime} \in T\left(\Sigma_{\theta}\right)$ ):

$$
t \stackrel{\tau}{\rightarrow} v \stackrel{a}{\rightarrow} v^{\prime}, \forall b>a v^{b} \nrightarrow, \text { and } u \equiv \theta\left(v^{\prime}\right)
$$

which is case (2) for $\theta$ in the Structuring Lemma. 
With the Structuring Lemma it is rather straightforward, but unpleasantly lengthy, to prove the soundness of the axioms.

THEOREM 10.3 (SOUNDNESS OF BPA $\left.{ }_{\delta \epsilon \tau}^{\theta}\right)$. Let $t, u \in T\left(\Sigma_{\theta}\right)$ :

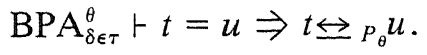

Proof. We must show that $R_{\mathrm{BPA}_{\delta \epsilon \tau}^{\theta}} \subseteq \leftrightarrow_{P_{\theta}}$ where $R_{\mathrm{BPA}_{\delta \epsilon \tau}^{\theta}}$ is the smallest congruence relation containing $(t, u)$ if $t=u$ is an instance of an axiom in $\mathrm{BPA}_{\delta \epsilon \tau}^{\theta}$. As by Theorem $8.13 \leftrightarrow_{P_{\theta}}$ is also a congruence relation, it is sufficient to show that

$$
t=u \text { is an instance of an axiom in } \mathrm{BPA}_{\delta \epsilon \tau}^{\theta} \Rightarrow t \leftrightarrow_{P_{\theta}} u .
$$

Suppose $t=u$ is an instance of an axiom. We will only consider axiom P3. All other axioms can be dealt with in the same way. Hence, $t \equiv a t^{\prime} \triangleleft \tau u^{\prime}, u \equiv$ $a t^{\prime} \triangleleft u^{\prime}\left(t^{\prime}, u^{\prime} \in T\left(\Sigma_{\theta}\right)\right)$ and $\neg(a<\tau)$. In order to show that $a t^{\prime} \triangleleft \tau u^{\prime} \leftrightarrow P_{\theta}$ $a t^{\prime} \triangleleft u^{\prime}$, it suffices to show that if $a t^{\prime} \triangleleft \tau u^{\prime} \stackrel{b}{\rightarrow} v,\left(b \in A_{\theta}\right)$ then $a t^{\prime} \triangleleft u^{\prime} \stackrel{\vec{b}}{\rightarrow} v$ and vice versa, $a t^{\prime} \triangleleft u^{\prime} \stackrel{b}{\rightarrow} v$ implies $a t^{\prime} \triangleleft \tau u^{\prime} \stackrel{b}{\rightarrow} v$. So suppose $a t^{\prime} \triangleleft \tau u^{\prime} \stackrel{b}{\rightarrow} v$. By the Structuring Lemma, one of the following cases must hold:

(1) $a t^{\prime} \stackrel{b}{\rightarrow} v, b \not \equiv \vee$ and $\forall c>b \tau u^{\prime} \stackrel{c}{\rightarrow}$,

(2) $a t^{\prime} \stackrel{\tau}{\rightarrow} t^{\prime \prime}, t^{\prime \prime} \stackrel{b}{\rightarrow} v$ and $b \not \equiv \vee$ for some $t^{\prime \prime} \in T\left(\Sigma_{\theta}\right)$,

(3) $a t^{\prime} \stackrel{\vee}{\rightarrow} v$ and $b \equiv \vee$.

Note that case (3) is impossible. So either case (1) or case (2) must hold. If case (2) holds, it is immediately clear that $a t^{\prime} \triangleleft u^{\prime} \stackrel{\tau}{\rightarrow} t^{\prime \prime}$ and $t^{\prime \prime} \stackrel{b}{\rightarrow} v$. Therefore, $a t^{\prime} \triangleleft u^{\prime} \stackrel{b}{\rightarrow} v$. If case (1) holds, then $\forall c>b u^{\prime}{ }^{c} \rightarrow$. If this were not the case, that is, $\exists c>b u^{\prime} \stackrel{c}{\rightarrow} u^{\prime \prime}$, then $\tau u^{\prime} \stackrel{c}{\rightarrow} u^{\prime \prime}$ contradicting that $\forall c>b \tau u^{\prime} \nrightarrow$ †. Hence, $a t^{\prime}$ $\triangleleft u^{\prime} \stackrel{b}{\rightarrow} v$.

The other implication can be proved likewise.

We now show completeness of the axioms. This is done in three stages. First the class of basic terms is introduced. This class is a subset of all closed $\Sigma_{\theta}$-terms, but it is still powerful enough to denote all recursion free processes. This is in fact shown in Lemma 10.6 .

Then operational characteristics are linked to the syntactic forms of terms using the operational soundness and completeness lemmas. In the last lemma, all results are gathered together and completeness is shown.

Definition 10.4. The set of basic terms is the smallest subset of $T\left(\Sigma_{\theta}\right)$ satisfying:

- $\delta$ and $\epsilon$ are basic terms,

- if $t$ is a basic term, then at ( $\left.a \in A c t_{\tau}\right)$ is a basic term,

- if $t, t^{\prime}$ are basic terms, then $t+t^{\prime}$ is a basic term.

Note that $a \epsilon$ and $a \epsilon+b \delta$ are basic terms but $a$ and $(a+b) c$ are not.

LEMMA 10.5. Let $t, t^{\prime}$ be basic terms. Then there is a basic term $u$ such that:

(1) $\mathrm{BPA}_{\delta \epsilon \tau}^{\theta} \vdash t \square t^{\prime}=u(\square=+, \cdot, \triangleleft)$, 
(2) $\mathrm{BPA}_{\delta \epsilon \tau}^{\theta} \vdash \square(t)=u\left(\square=\tau_{I}, \theta\right)$.

Proof. As an example we show the proof for $\triangleleft$. For a basic term $t$ define \#t as the number of function names in $t$. Define the depth of a term $t \triangleleft t^{\prime}$ with $t, t^{\prime}$ basic terms by ( $\omega$ is the first infinite ordinal):

$$
D\left(t \triangleleft t^{\prime}\right)=\omega \cdot \# t^{\prime}+\# t .
$$

We prove this case with induction on $D\left(t \triangleleft t^{\prime}\right)$. Distinguish the following cases:

$$
\begin{aligned}
& t=\epsilon, \delta \text { Apply PE1 or PD1. } \\
& t^{\prime}=\epsilon, \delta \text { Apply PE2 or PD2. } \\
& t=a u_{1}, t^{\prime}=b u_{2} \text {, Apply P1 or P2. } \\
& (b \not \equiv \tau) \\
& t=a u_{1}, \quad \text { Apply P1 if } a<\tau \text {. If } \neg(a<\tau) \text {, then BPA }{ }_{\delta \in \tau}^{\theta} \vdash a u_{1} \triangleleft \\
& t^{\prime}=\tau u_{2} \quad \tau u_{2} \stackrel{P 3}{=} a u_{1} \triangleleft u_{2} \text {. As } D\left(a u_{1} \triangleleft u_{2}\right)<D\left(a u_{1} \triangleleft \tau u_{2}\right) \text {, it } \\
& \text { follows with induction that } \mathrm{BPA}_{\delta \epsilon \tau}^{\theta} \vdash a u_{1} \triangleleft u_{2}=v \text { for } \\
& \text { some basic term } v \text {. } \\
& t^{\prime}=u_{1}+u_{2} \quad \text { We have that } t \triangleleft t^{\prime} \equiv t \triangleleft\left(u_{1}+u_{2}\right) \stackrel{P 4}{=}\left(t \triangleleft u_{1}\right) \triangleleft u_{2} \text {. } \\
& \text { As } D\left(t \triangleleft u_{1}\right)<D\left(t \triangleleft\left(u_{1}+u_{2}\right)\right) \text {, it follows that } \\
& \mathrm{BPA}_{\delta \epsilon \tau}^{\theta}+t \triangleleft u_{1}=v \text { for some basic term } v \text {. As } D(v \triangleleft \\
& \left.u_{2}\right)<D\left(t \triangleleft\left(u_{1}+u_{2}\right)\right) \text {, it follows that } \mathrm{BPA}_{\delta \in \tau}^{\theta} \vdash(t \triangleleft \\
& \left.u_{1}\right) \triangleleft u_{2}=v \triangleleft u_{2}=v^{\prime} \text { for some basic term } v^{\prime} \text {. } \\
& t=u_{1}+u_{2} \quad \text { It follows that } t \triangleleft t^{\prime} \equiv\left(u_{1}+u_{2}\right) \triangleleft t^{\prime} \stackrel{P 5}{=} u_{1} \triangleleft t^{\prime}+u_{2} \\
& \triangleleft t^{\prime} \text {. As } D\left(u_{1} \triangleleft t^{\prime}\right)<D\left(\left(u_{1}+u_{2}\right) \triangleleft t^{\prime}\right) \text { and } D\left(u_{2} \triangleleft\right. \\
& \left.t^{\prime}\right)<D\left(\left(u_{1}+u_{2}\right) \triangleleft t^{\prime}\right) \text {, there are basic terms } v, v^{\prime} \\
& \text { such that } \mathrm{BPA}_{\delta \epsilon \tau}^{\theta} \vdash u_{1} \triangleleft t^{\prime}=v \text { and } \mathrm{BPA}_{\delta \epsilon \tau}^{\theta} \vdash u_{2} \triangleleft t^{\prime}= \\
& v^{\prime} \text {. Hence, } \mathrm{BPA}_{\delta \epsilon \tau}^{\theta} \vdash t \triangleleft t^{\prime}=v+v^{\prime} \text {. }
\end{aligned}
$$

LEMMA 10.6. Let $t \in T\left(\Sigma_{\theta}\right)$ be a recursion free term. Then there is a basic term u such that:

$$
\mathrm{BPA}_{\delta \epsilon \tau}^{\theta} \vdash t=u .
$$

Proof. Apply induction on the structure of $t$. If $t \equiv \epsilon, \delta, a\left(\in A c t_{\tau}\right)$ then the basic terms are respectively: $\epsilon, \delta$ and $a \epsilon$. If $t \equiv t_{1} \square t_{2}(\square \equiv+, \cdot, \triangleleft)$, it follows with induction that $t_{1}$ and $t_{2}$ are provably equal to basic terms $u_{1}, u_{2}$. Then Lemma 10.5 yields $\mathrm{BPA}_{\delta \epsilon \tau}^{\theta} \vdash u_{1} \square u_{2}=u$ with $u$ a basic term. For the unary operators $\theta$ and $\tau_{I}$, a similar argument can be applied.

The following notation is an abbreviation that turns out to be useful.

Notation 10.7 (Summand Inclusion). We write $t \subseteq t^{\prime}$ for $t+t^{\prime}=t^{\prime}$.

The following lemmas relate summand inclusion to the operational rules in Table I. They state that if a process $t$ can perform an $a$-step $\left(t \stackrel{a}{\rightarrow} t^{\prime}\right)$ then it is provable that $a t^{\prime}$ is a summand of $t$. A weak variant of the converse also holds.

LEMma 10.8 (OPERATIONAL SOUNDNESS). Let $t, t^{\prime} \in T\left(\Sigma_{\theta}\right)$ be recursion free terms and let $a \in A c t_{\tau}$ : 


$$
\begin{gathered}
\mathrm{BPA}_{\delta \epsilon \tau}^{\theta} \vdash a \cdot t^{\prime} \subseteq t \Rightarrow \exists t^{\prime \prime}: t \stackrel{a}{\rightarrow} t^{\prime \prime} \text { and } t^{\prime \prime} \overleftrightarrow{\leftrightarrow}_{P_{\theta}} t^{\prime}, \\
\mathrm{BPA}_{\delta \epsilon \tau}^{\theta} \vdash \epsilon \subseteq t \Rightarrow \exists t^{\prime}: t \stackrel{\vee}{\rightarrow} t^{\prime} .
\end{gathered}
$$

ProOF. Directly using the Soundness Theorem 10.3.

Lemma 10.9 (Operational COMPLeteness). Let $t, t^{\prime} \in T\left(\Sigma_{\theta}\right)$ be recursionfree and $\theta, \triangleleft$-free terms and let $a \in A c t_{\tau}$ :

$$
\begin{aligned}
& t \stackrel{a}{\rightarrow} t^{\prime} \Rightarrow \mathrm{BPA}_{\delta \epsilon \tau}^{\theta} \vdash a t^{\prime} \subseteq t, \\
& t \stackrel{\vee}{\rightarrow} t^{\prime} \Rightarrow \mathrm{BPA}_{\delta \epsilon \tau}^{\theta} \vdash \epsilon \subseteq t .
\end{aligned}
$$

Proof. Straightforward induction on the proof of $t \stackrel{a}{\rightarrow} t^{\prime}$ and $t \stackrel{\vee}{\rightarrow} t^{\prime}$ from $\operatorname{Strip}\left(P_{\theta}, \rightarrow_{P_{\theta}}\right)$.

LEMMA 10.10. Let $t$ be a basic term. If $t \stackrel{a}{\rightarrow} t^{\prime}(a \in A c t)$, then $t^{\prime} \equiv \epsilon \cdot u$ or $t^{\prime} \equiv$ $\tau \cdot u$ for some basic term $u$. Moreover, $t^{\prime}$ contains at most as many function names as $t$.

Proof. Use induction on the proof of $t \stackrel{a}{\rightarrow} t^{\prime}$ from $\operatorname{Strip}\left(P_{\theta}, \rightarrow_{P_{\theta}}\right)$.

Notation 10.11. Let $t, u \in T\left(\Sigma_{\theta}\right)$ be recursion free. $t \rightrightarrows_{P_{\theta}} u$ stands for: $t \stackrel{a}{\rightarrow} t^{\prime}$ implies $\exists u^{\prime} u \stackrel{a}{\rightarrow} u^{\prime}$ and $t^{\prime} \overleftrightarrow{\leftrightarrow}_{P_{\theta}} u^{\prime}$. Note that this condition resembles clause 1 in the definition of bisimulation.

LEMMA 10.12. Let $t$ and $u$ be basic terms over $B P A_{\delta \epsilon \tau}^{\theta}$. Then:

(1) If $t \rightarrow_{P_{\theta}} u$, then $\mathrm{BPA}_{\delta \epsilon \tau}^{\theta} \vdash t \subseteq u$,

(2) If $t \leftrightarrow_{P_{\theta}} u$, then $\mathrm{BPA}_{\delta \epsilon \tau}^{\theta} \vdash t=u$.

Proof. We use induction on the number of function names in $t$ and $u$, that is, \#t $+\# u$. The proof employs the operational soundness and completeness lemmas.

Basis. First (1) is proved. Suppose that $t \equiv \epsilon$ and $u \in T\left(\Sigma_{\theta}\right) . \epsilon \rightarrow_{P_{\theta}} u \Rightarrow$ $u \stackrel{\vee}{\rightarrow} u^{\prime} \Rightarrow \mathrm{BPA}_{\delta \epsilon \tau}^{\theta} \vdash \epsilon \subseteq u$. Suppose $t \equiv \delta$. This case is trivial using axiom A6. In case (2) $t \leftrightarrow_{P_{\theta}} u$ implies $t \rightrightarrows_{P_{\theta}} u$ and $u \rightarrow_{P_{\theta}} t$, so it follows by (1) that $\mathrm{BPA}_{\delta \epsilon \tau}^{\theta} \vdash t \subseteq$ $u$ and $\mathrm{BPA}_{\delta \epsilon \tau}^{\theta} \vdash u \subseteq t$. Hence, $\mathrm{BPA}_{\delta \epsilon \tau}^{\theta} \vdash t=t+u=u+t=u$.

Induction. First consider (1). Suppose $t \equiv\left(t_{1}+t_{2}\right) \underset{\Rightarrow}{\rightarrow} p_{\theta} u$. This implies that $t_{1} \rightarrow_{P_{\theta}} u$ and $t_{2} \rightrightarrows_{P_{\theta}} u$. Using (1) inductively yields: $\mathrm{BPA}_{\delta \epsilon \tau}^{\theta} \vdash t_{1} \subseteq u$ and $\mathrm{BPA}_{\delta \epsilon \tau}^{\theta}$ $\vdash t_{2} \subseteq u$. Now using axiom A1 leads to $\mathrm{BPA}_{\delta \epsilon \tau}^{\theta} \vdash t_{1}+t_{2} \subseteq u$.

Now suppose that $t \equiv a t_{1} \rightarrow_{P_{\theta}} u$. Note that \#t $t_{1}<\# t$. There is a $t_{2}$ (e.g., $\epsilon t_{1}$ )

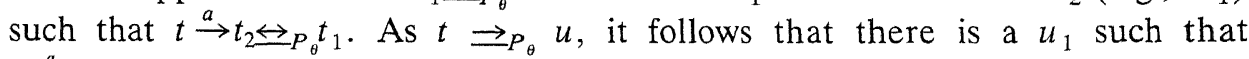
$u \stackrel{a}{\rightarrow} u_{1}, t_{1} \overleftrightarrow{\leftrightarrow}_{P_{\theta}} t_{2} \leftrightarrow_{P_{\theta}} u_{1}$. By Lemma 10.10, $u_{1}$ is a basic term and $\# u_{1} \leq \# u$. With the induction hypothesis, conclude that $\mathrm{BPA}_{\delta \epsilon \tau}^{\theta} \vdash t_{1}=u_{1}$. By operational completeness, it follows that $\mathrm{BPA}_{\delta \epsilon \tau}^{\theta} \vdash a u_{1} \subseteq u$. Therefore, $\mathrm{BPA}_{\delta \epsilon \tau}^{\theta} \vdash a t_{1} \subseteq u$.

In case (2), $t \leftrightarrow_{P_{\theta}} u$ implies $t \rightrightarrows_{P_{\theta}} u$ and $u \rightrightarrows_{P_{\theta}} t$, so it follows by (1) that $\mathrm{BPA}_{\delta \epsilon \tau}^{\theta} \vdash t \subseteq u$ and $\mathrm{BPA}_{\delta \epsilon \tau}^{\theta} \vdash u \subseteq t$. Hence, $\mathrm{BPA}_{\delta \epsilon \tau}^{\theta^{\theta}} \vdash t=t+u=u+t=u$.

THEOREM 10.13 (COMPLETENESS OF BPA ${ }_{\delta \epsilon \tau}^{\theta}$ ). Let $t, u \in T\left(\Sigma_{\theta}\right)$ be recursion free. It holds that: 


$$
t \leftrightarrow_{P_{\theta}} u \Rightarrow \mathrm{BPA}_{\delta \epsilon \tau}^{\theta} \vdash t=u
$$

Proof. Suppose $t \leftrightarrow p_{\theta} u$. Then there are basic terms $t^{\prime}$ and $u^{\prime}$ that are provably equivalent to $t$ and $u$. With soundness, it follows that $t^{\prime} \leftrightarrow_{P_{\theta}} u^{\prime}$. An application of Lemma 10.12 yields $\mathrm{BPA}_{\delta \epsilon \tau}^{\theta} \vdash t^{\prime}=u^{\prime}$ and thus $\mathrm{BPA}_{\delta \epsilon \tau}^{\theta^{\theta}} \vdash$ $t=u$.

Appendix $A$. The relation between TSSs and logic programs

Throughout this paper, techniques from logic programming are applied to TSSs. This raises the question of whether TSSs can be viewed as logic programs. It appears that there indeed exists a straightforward translation from TSSs to logic programs.

Definition A.1. Let $P=(\Sigma, A, R)$ be a TSS. We define the translation $\mathscr{L}$ as:

for every positive literal $t \stackrel{a}{\rightarrow} t^{\prime}: \mathscr{L}\left(t \stackrel{a}{\rightarrow} t^{\prime}\right)=\operatorname{transition}\left(t, a, t^{\prime}\right)$,

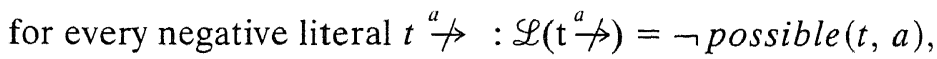

for every rule $r \in R: \mathscr{L}(r)=\mathscr{L}(\operatorname{conc}(r)) \leftarrow \mathscr{L}(\operatorname{prem}(r)){ }^{2}$

and finally

$$
\mathscr{L}(P)=\mathscr{L}(R) \cup\{\text { possible }(T, A) \leftarrow \operatorname{transition}(T, A, U)\}
$$

where $T, A$, and $U$ are variables.

For an introduction in logic programming, we refer to Lloyd [1987]; for negation in logic programming, we refer to Apt and Bol [1994]. We must point out some small differences between the two formalisms.

First of all, logic programs are usually untyped, whereas a TSS $P=(\Sigma, A, R)$ has clearly two types, namely terms (from $T(\Sigma)$ ) and labels (from $A$ ). Thus, the translation $\mathscr{L}(P)$ must also be treated as a typed program, its Herbrand base being

$$
\begin{aligned}
H B_{P}= & \left\{\text { transition }\left(t, a, t^{\prime}\right) \mid t, t^{\prime} \in T(\Sigma), a \in A\right\} \\
& \cup\{\text { possible }(t, a) \mid t \in T(\Sigma), a \in A\} .
\end{aligned}
$$

Secondly, a traditional logic program consists of a finite set of finite clauses. A TSS may have an infinite number of rules and each rule may have infinitely many premises. The main reason for this is that, in TSSs, only variables ranging over terms are used, and no variables ranging over labels. Thus, instead of one rule like

$$
\frac{x \stackrel{z}{\rightarrow} x^{\prime}}{x+y \stackrel{z}{\rightarrow} x^{\prime}},
$$

this rule must be incorporated for every action $z$ separately. Usually rule schemes with meta-variables ranging over $A$ are given, as, in this case, rule R3.1 of the

${ }^{2}$ As usual $\mathscr{L}(X)$ abbreviates $\{\mathscr{L}(x) \mid x \in X\}$. 
running example. Translating a TSS yields a possibly infinite set of possibly infinite clauses. Of course, having an infinite number of clauses is not a problem: the set of ground instances of clauses from a traditional logic program is normally infinite as well. Having infinitely many premises seems harmless too.

In order to formulate the intended correspondencies between the TSS $P$ and the logic program $\mathscr{L}(P)$, we also need a translation on the semantical level, that is between transitions relations and (well-typed) Herbrand interpretations.

Definition A.2. Let $\rightarrow$ be a transition relation.

$$
\begin{aligned}
M(\rightarrow)= & \left\{\operatorname{transition}\left(t, a, t^{\prime}\right) \mid t \stackrel{a}{\rightarrow} t^{\prime} \in \rightarrow\right\} \\
& \cup\left\{\operatorname{possible}(t, a) \mid \exists t^{\prime}: t \stackrel{a}{\rightarrow} t^{\prime} \in \rightarrow\right\} .
\end{aligned}
$$

According to this definition, only interpretations $M$ satisfying for all $t$ and $a$ :

$$
\text { possible }(t, a) \in M \text { iff } \exists t^{\prime}: \text { transition }\left(t, a, t^{\prime}\right) \in M
$$

are translations of a transition relation. The clause possible $(T, A) \leftarrow$ transi$\operatorname{tion}(T, A, U)$ is obviously incorporated in the translation of every TSS to enforce this property. As long as only supported models of the resulting logic programs are considered, the addition of this clause is indeed sufficient. (See Figure 2.) The following example shows that a weaker choice of semantics (in this case, minimal models) can produce certain anomalous models.

Example A.3. Consider the TSS $P$ with one constant $c$ and one unary function $f$, one action $a$ and the following rules:

$$
\frac{c \stackrel{a}{\rightarrow}}{c \stackrel{a}{\rightarrow} c} \quad \frac{c \stackrel{a}{\rightarrow} x}{c \stackrel{a}{\rightarrow} f(x)} .
$$

For all $n \geq 0$, the transition relation $\left\{c \stackrel{a}{\rightarrow} f^{i}(c) \mid i \geq n\right\}$ is a model of $P$; $P$ has no other models. As $n$ increases, the model decreases (with respect to $\subseteq$ ), thus $P$ has no minimal model. Now consider

$$
\begin{aligned}
\mathscr{L}(P)= & \{\operatorname{transition}(c, a, c) \leftarrow \neg \text { possible }(c, a) \\
& \operatorname{transition}(c, a, f(X)) \leftarrow \operatorname{transition}(c, a, X) \\
& \operatorname{possible}(T, A) \leftarrow \operatorname{transition}(T, A, U)\} .
\end{aligned}
$$

The corresponding models are (for all $n \geq 0$ ):

$$
\left\{\operatorname{transition}\left(c, a, f^{i}(c)\right) \mid i \geq n\right\} \cup\{\text { possible }(c, a)\} .
$$

But $\mathscr{L}(P)$ has one more model, namely just $\{$ possible $(c, a)\}$, which is the least model of $\mathscr{L}(P)$, but not supported by $\mathscr{L}(P)$.

As we concentrate on the stable and well-founded model semantics, which generate only supported models, anomalous models will no longer arise.

In the rest of this section, we establish the relationships between TSSs and their translations into logic programs. For the definitions regarding logic programming we refer to Apt and Bol [1994]. As these definitions are always similar 


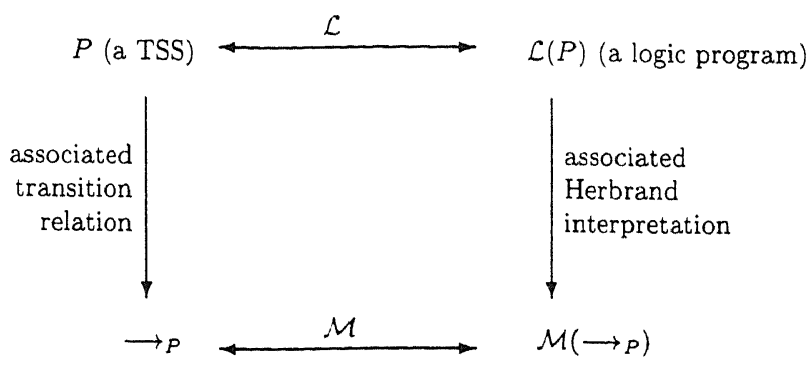

FIG. 2. The relation between TSSs and logic programs.

to the definitions regarding TSSs as presented in this paper, it is straightforward to prove the following propositions.

Proposition A.4. Let $P$ be a TSS.

$-P$ is positive iff $\mathscr{L}(P)$ is positive.

- $P$ is stratified iff $\mathscr{L}(P)$ is locally stratified (see Przymusinski [1987]).

For a positive logic program $P, M_{P}$ denotes its least Herbrand model.

Proposition A.5. Let $P$ be a TSS and $\rightarrow$ be a transition relation.

$\longrightarrow \rightarrow$ is stable for $P$ iff $\mathcal{M}(\rightarrow)$ is a stable model [Gelfond and Lifschitz 1988] of $\mathscr{L}(P)$.

In particular,

- if $P$ is positive, then $\mathcal{M}\left(\rightarrow_{P}\right)=M_{\mathscr{L}(P)}$ and

- if $P$ is stratified, then $\mathcal{M}\left(\rightarrow_{P}\right)$ is the unique perfect model [Przymusinski 1987] of $\mathscr{L}(P)$.

Many slightly different, but equivalent, definitions of well-founded models for logic programs have been given. Here we follow Przymusinski [1989].

Definition A.6 (Well-Founded Model). Let $P$ be a logic program.

-A 3-valued interpretation for $P$ is a pair $I=\langle T, F\rangle$, where $T$ and $F$ are subsets of the Herbrand base $H B_{P}$ (but not necessarily $T \cap F=\emptyset$ ).

- Let $A$ be a ground atom. Then, $\langle T, F\rangle \vDash A$ iff $A \in T$ and $\langle T, F\rangle \vDash \neg A$ iff $A \in F$.

$-T_{P}(I)=\left\{A \in H B_{P} \mid\right.$ there exists a clause $A \leftarrow L_{1}, \ldots, L_{n} \in \operatorname{ground}(P)$ such that $I \vDash L_{1}$ and $\cdots$ and $\left.I \vDash L_{n}\right\} . F_{P}(I)=\left\{A \in H B_{P} \mid\right.$ for every clause $A \leftarrow L_{1}, \ldots, L_{n} \in \operatorname{ground}(P): I \vDash \neg L_{1}$ or $\cdots$ or $\left.I \vDash \neg L_{n}\right\}$.

(If $L$ is a negative literal $\neg B$, then $\neg L$ denotes $B$.)

$T_{P}(I)$ defines the ground atoms that are immediately true given $P$ and $I, F_{P}(I)$ defines the ground atoms that are immediately false.

-Let $T, F \subseteq H B_{P}$ and let $I$ be a 3-valued interpretation for $P$.

$\mathscr{T}_{I}(T)=T_{P}(I \cup\langle T, \emptyset\rangle)$.

$\mathscr{F}_{I}(F)=F_{P}(I \cup\langle\emptyset, F\rangle)$.

$\oiint_{P}(I)=I \cup\left\langle\cup_{n<\omega} \mathscr{T}_{I}^{n}(\emptyset), \cap_{n<\omega} \mathscr{F}_{I}^{n}\left(H B_{P}\right)\right\rangle . \quad$ (Note: $\cup$ denotes pointwise union.) 
$\mathscr{I}_{P}(I)$ defines the ground atoms that are certainly true respectively false given $P$ and $I$.

-For a limit ordinal $\alpha: I_{\alpha}=\cup_{\beta<\alpha} I_{\beta}$ (in particular: $I_{0}=\langle\emptyset, \emptyset\rangle$ ).

For a successor ordinal $\alpha+1: I_{\alpha+1}=\Phi_{P}\left(I_{\alpha}\right)$.

- Let $\delta$ be the smallest countable ordinal such that $I_{\delta}=\mathscr{I}\left(I_{\delta}\right)$. Then $I_{\delta}$ is the well-founded (partial) model of $P$. If $I_{\delta}$ is 2-valued, that is, $I_{\delta}=\langle T, F\rangle$ is a partitioning of $H B_{P}$, then $I_{\delta}$ is the well-founded (complete) model of $P$.

An alternative definition of the well-founded model, based on the reduction of logic programs, can also be given.

Definition A.7. Let $P$ be a logic program and $I$ a 3-valued interpretation for $P$. Then:

$$
\operatorname{Reduce}(P, I)=\cup_{C \in \operatorname{ground}(P)} \operatorname{Reduce}(C, I),
$$

where

$$
\operatorname{Reduce}(A \leftarrow S, I)= \begin{cases}\emptyset & \text { if for some literal } L \in S: I \vDash \neg \\ \left\{A \leftarrow S^{\prime}\right\} & \text { otherwise, where } S^{\prime}=\{L \in S \mid I \not L\} .\end{cases}
$$

Furthermore:

True $(P)=\{A \leftarrow S \in P \mid S$ contains only positive literals $\}$ and

$\operatorname{Pos}(P)=\left\{A \leftarrow S^{\prime} \mid\right.$ there is a clause $A \leftarrow S \in P$ such that

$S^{\prime}$ is the set of positive literals in $S$ \}.

Lemma A.8. Let $P$ be a logic program and I a 3-valued interpretation for $P$.

$$
\begin{aligned}
\cup_{n<\omega} \mathscr{T}_{I}^{n}(\emptyset) & =M_{\text {True }(\operatorname{Reduce}(P, I))}, \\
\bigcap_{n<\omega} \mathscr{F}_{I}^{n}\left(H B_{P}\right) & =H B_{P}-M_{\operatorname{Pos}(\operatorname{Reduce}(P, I))} .
\end{aligned}
$$

Thus, an alternative definition of the well-founded (partial) model of a logic program is obtained by replacing $\cup_{n<\omega} \mathscr{T}_{I}^{n}(\emptyset)$ by $\operatorname{MTrue}(\operatorname{Reduce}(P, I))$ and $\cap_{n<\omega} \mathscr{F}_{I}^{n}\left(H B_{P}\right)$ by $H B_{P}-M_{P o s(\operatorname{Reduce}(P, I))}$ in Definition A.6. The proof of Lemma A.8 is beyond the scope of this paper.

Using this alternative definition, it is straightforward to link the reduction of a TSS $P$ and the sequence of interpretations leading to the well-founded (partial) model of $\mathscr{L}(P)$.

Lemma A.9. Let $P=(\Sigma, A, R)$ be a TSS and let $\rightarrow_{\text {true }}, \rightarrow_{\text {pos }} \subseteq \operatorname{Tr}(\Sigma, A)$. Then:

$-\mathscr{L}(\operatorname{True}(P))=\operatorname{True}(\mathscr{L}(P))$,

$-\mathscr{L}(\operatorname{Pos}(P))=\operatorname{Pos}(\mathscr{L}(P))$,

$-\mathscr{L}\left(\operatorname{Reduce}\left(P, \rightarrow_{\text {true }}, \rightarrow_{\text {pos }}\right)\right)=\operatorname{Reduce}\left(\mathscr{L}(P),\left\langle M\left(\rightarrow_{\text {true }}\right), H B_{P}-\mathcal{M}\left(\rightarrow_{\text {pos }}\right)\right\rangle\right)$.

THEOREM A.10. Let $P$ be a TSS. Let for all ordinals $\alpha, I_{\alpha}$ be defined with respect to $\mathscr{L}(P)$ as in Definition A.6. Then:

$$
\mathscr{L}\left(\operatorname{Red}^{\alpha}(P)\right)=\operatorname{Reduce}\left(\mathscr{L}(P), I_{\alpha}\right)
$$




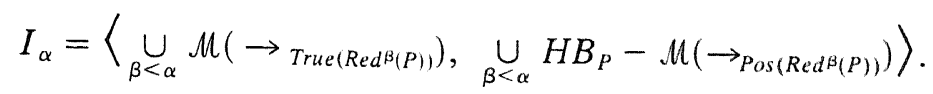

ProOF. Straightforward.

COROLLARY A.11. Let $P$ be a TSS. If $\mathscr{L}(P)$ has a well founded complete model $I_{\alpha}$, then $\operatorname{Red}^{\alpha}(P)$ is a positive TSS and $I_{\alpha}=M\left(\rightarrow_{\operatorname{Red}^{\alpha}(P)}\right)$. If $\operatorname{Red}^{\alpha}(P)$ is a positive TSS then $\mathscr{L}(P)$ has a well founded complete model $I_{\alpha+1}=\mathcal{M}\left(\rightarrow_{\operatorname{Red}^{\alpha}(P)}\right)$.

The change from $\alpha$ to $\alpha+1$ in the second implication is caused by the fact that it is possible that at the end of the iteration first the interpretation $I_{\alpha}$ becomes 2 -valued (making $\operatorname{Reduce}\left(\mathscr{L}(P), I_{\alpha}\right)$ positive), but also that a partial $I_{\alpha}$ results in a positive $\operatorname{Reduce}\left(\mathscr{L}(P), I_{\alpha}\right)$, in which case only $I_{\alpha+1}$ is 2 -valued.

Apart from its theoretical merits, the translation of TSSs into logic programs has also more practical implications. For logic programs interpreters and compilers are available. Thus, in order to find out whether a term $t$ can perform an $a$-step according to $\rightarrow_{P}$, the TSS $P$ is translated into the logic program $\mathscr{L}(P)$, and the query $\leftarrow$ transition $(t, a, X)$ is presented to it.

This poses only one problem: The depth-first strategy of most programming systems tends to result in nontermination of the program without finding all solutions. The rules R9.2 and R9.3 of the running example are typically rules leading to nontermination. More advanced query evaluation strategies, such as SLG-resolution [Chen and Warren 1993] might partly solve the problem, but as even for positive TSSs $\rightarrow_{P}$ need not be recursive, nontermination can never be ruled out completely. In the presence of negation $\rightarrow_{P}$ is in general not even recursively enumerable.

Thus, the translation into logic programming cannot be expected to produce the associated transition relation as a whole. But in our opinion the interactive use of a logic programming environment for proving that a certain transition holds (or does not hold) is an attractive alternative to generating this proof by hand, especially for larger TSSs.

On the bright side is that for pure TSSs (see Definition 8.3) the problem of floundering (the necessity to resolve a non-ground negative literal) does not occur for queries of the form $\leftarrow$ transition $(t, a, X)$ (with $t \in T(\Sigma)$ and $a \in A$ ). This can be shown by annotating the program (in the sense of Dembinski and Maluszynski [1985]) by transition ( $\downarrow, \downarrow, \uparrow)$ and possible $(\downarrow, \downarrow)$, meaning that the first and second argument of both predicates are considered to be input, and the third argument of transition is output. (Due to the fact that TSSs have no variables ranging over labels, the annotations of the second (label) arguments are inessential.)

Proposition A.12. Let $P=(\Sigma, A, R)$ be a TSS. Let $t \in T(\Sigma)$ and $a \in A$. If $P$ is pure then $\mathscr{L}(P) \cup\{\leftarrow$ transition $(t, a, X)\}$ is well formed (see Dembinski and Maluszynski [1985]) with respect to the above annotation.

The well-formedness of a logic program and a query implies that during the computation every predicate is called with ground terms on its input arguments. In particular, every call $\neg$ possible $(t, a)$ will be ground. In a more general setting, this annotation gives insight in the data-flow of the act of proving transitions from pure TSSs. 
ACKNowledgments. We thank Krzysztof Apt, Jos Baeten, Jan Bergstra, Rob van Glabbeek, Alban Ponse, Chris Verhoef and Fer-Jan de Vries for their valuable comments.

\section{REFERENCES}

ACETO, L. 1994. Deriving complete inference systems for a class of GSOS languages generating regular behaviours. In Proceedings of CONCUR '94, B. Jonsson and J. Parrow, eds. Lecture Notes in Computer Science, vol. 836. Springer-Verlag, New York, pp. 449-464.

Aceto, L., Bloom, B., and VaAndrager, F. W. 1994. Turning SOS rules into equations. Inf. Comput. 111, 1, 1-52.

ACETO, L., AND INGÓLFSDÓTTIR, A. 1994/1995. CPO models for a class of GSOS languages. In TAPSOFT '95, 6th International Joint Conference on Theory and Practice of Software Development, (Århus, Denmark), P. D. Mosses, M. Nielsen, and M. I. Schwartzbach, eds. Lecture Notes in Computer Science, vol. 915. Springer-Verlag, New York, 1995, pp. 439-453. (Full version appeared as CPO Models for GSOS Languages-Part I: Compact GSOS Languages, BRICS research report RS-94-10, Department of Mathematics and Computer Science, Aalborg University, 1994.)

APT, K. R., AND BoL, R. N. 1994. Logic programming and negation: A survey. J. Logic Program. $19-20,9-71$.

Baeten, J. C. M., and Bergstra, J. A. 1988. Processen en procesexpressies. Informatie 30, 3, 177-248 (in Dutch).

BAeten, J. C. M., Bergstra, J. A., AND Klop, J. W. 1986. Syntax and defining equations for an interrupt mechanism in process algebra. Fund. Inf. IX, 2, 127-168.

BAETEN, J. C. M., AND VeRhoef, C. 1993. A congruence theorem for structured operational semantics with predicates. In Proceedings of CONCUR '93, E. Best, ed. Lecture Notes in Computer Science, vol. 715. Springer-Verlag, New York, pp. 477-492.

Baeten, J. C. M., AND Weisland, W. P. 1990. Process Algebra. Cambridge Tracts in Theoretical Computer Science 18. Cambridge University Press, Cambridge, Mass.

Best, E., And Koutny, M. 1990. Partial order semantics of priority systems. Tech. Rep. 6/90. Univ. Hildesheim, Institut für Informatik.

BLoom, B. 1995. Structural operational semantics for weak bisimulations. Theoret. Comput. Sci. $146,25-68$.

Bloom, B., Istrail, S., AND MeYer, A. R. 1995. Bisimulation can't be traced. J. ACM 42, 1 (Jan.), 232-268.

Bolognesi, T., Lucidi, F., and Trigila, S. 1990. From timed Petri nets to timed LOTOS. In Proceedings of the 10th IFIP WG6.1 International Symposium on Protocol Specification, Testing and Verification, (Ottawa, Ont., Canada). North-Holland, Amsterdam, The Netherlands, pp. 395-408.

Camilleri, J. 1989. An operational semantics for OCCAM. Int. J. Parall. Prog. 18, 5, 149-167.

CHEN, W., AND WARREN, D. S. 1993. Query evaluation under the well founded semantics. In Proceedings of the 12th ACM SIGACT-SIGMOD-SIGART Symposium on Principles of Database Systems (Washington, D. C., May 25-28). ACM, New York, pp. 168-179.

Cleaveland, R., And Hennessy, M. 1988. Priorities in process algebra. In Proceedings of the $3 r d$ Annual Symposium on Logic in Computer Science, (Edinburgh, Scotland). IEEE Computer Society Press, New York, pp. 193-202.

D'ARgenio, P. R. 1995. A general conservative extension theorem in process algebras with inequalities. In Proceedings of ACP'95, A. Ponse, C. Verhoef, and S. F. M. van Vlijmen, eds. Computing Science Reports 95-14, Department of Mathematics and Computer Science, Eindhoven University of Technology.

DEMBINSKI, P., AND MAeUSZYNSKI, J. 1985. And-parallelism with intelligent backtracking for annotate logic programs. In Proceedings of the 7th Symposium on Logic Programming, (Boston, Mass.). pp. 29-38.

Fokkink, W. J. And van Glabbeek, R. J. 1996. Ntyft/ntyxt rules reduce to ntree rules. Inf. Comput., to appear.

FoKKINK, W. J., AND VerhoEF, C. 1995. A conservative look at term deduction systems with variable binding. Computing Science Reports 95-28, Dept. of Mathematics and Computing Science, Eindhoven University.

van Gelder, A., Ross, K. AND SCHLIPF, J. S. 1988. Unfounded sets and well-founded semantics for general logic programs. In Proceedings of the 7th ACM SIGACT-SIGMOD-SIGART Symposium on Principles of Database Systems, (Austin, Texas, March 21-23). ACM, New York, pp. 221-230. 
Gelfond, M., AND Lifschitz, V. 1988. The stable model semantics for logic programming. In Proceedings of the 5th International Conference on Logic Programming, (Cambridge, Mass.), R. Kowalski and K. Bowen, eds. MIT Press, Cambridge, Mass., pp. 1070-1080.

VAN GlabBEEK, R. J. 1987. Bounded nondeterminism and the approximation induction principle in process algebra. In Proceedings of STACS 87, F. J. Brandenburg. G. Vidal-Naquet, and $\mathrm{M}$. Wirsing, eds. Lecture Notes in Computer Science, vol. 247. Springer-Verlag, New York, pp. $336-347$.

VAN Glabbeek, R. J. 1990. The linear-branching time spectrum. In Proceedings of CONCUR90, (Amsterdam, The Netherlands), J. C. M. Baeten and J. W. Klop, eds. Lecture Notes in Computer Science, vol. 458. Springer-Verlag, New York, pp. 278-297.

vaN GlabBeEK, R. J. 1993a. Full abstraction in structural operational semantics. In Proceedings of AMAST '93, Workshops in Computing. Springer-Verlag, New York, pp. 77-84.

VAN GlabBEEK, R. J. 1993b. The linear time-branching time spectrum II (The semantics of sequential systems with silent moves). In Proceedings of CONCUR93, E. Best, ed. Lecture Notes in Computer Science, vol. 715. Springer-Verlag, New York, pp. 66-81.

VAN GlabbeeK, R. J. 1995. The meaning of negative premises in transition system specifications II. Technical Note CS-95-16, Stanford University, Available by anonymous ftp at $\mathrm{ftp}: / /$ boole.stanford.edu/pub/DVI/negative.dvi.gz.

Groote, J. F. 1993. Transition system specifications with negative premises. Theoret. Comput. Sci. $118,2,263-299$.

Groote, J. F., AND VAANDRAger, F. W. 1992. Structured operational semantics and bisimulation as a congruence. Inf. Comput. 100, 2 (Oct.), 202-260.

Hennessy, M., AND Plotkin, G. D. 1979. Full abstraction for a simple programming language. In Proceedings of the 8th Symposium on Mathematical Foundations of Computer Science, J. Bečvár, ed. Lecture Notes in Computer Science, vol. 74. Springer-Verlag, New York, pp. 108-120.

Hennessy, M., AND REGAN, T. 1990. A temporal process algebra. Report 2/90, Computer Science Department, Univ. Sussex, Sussex, England.

HOWE, D. J. 1996. Proving congruence of bisimulation in functional programming languages. Inf. Comput. to appear.

ICHIKAWA, H., YAMANAKA, K., AND KATO, J. 1990. Incremental specifications in LOTOS. In Proceedings of the 10th IFIP WG6.1 International Symposium on Protocol Specification, Testing and Verification, (Ottowa, Ont., Canada), North-Holland, Amsterdam, The Netherlands, L. Logrippo, R. L. Probert, and H. Ural, eds. pp. 183-196.

JANICKI, R. 1987. A formal semantics for concurrent systems with a priority relation. Acta. Inf. 24, 33-55.

LANGERAK, R. 1989. A testing theory for LOTOS using deadlock detection. In Proceedings of the 9th IFIP WG6.1 International Symposium on Protocol Specification, Testing, and Verification, (Enschede), E. Brinksma, G. Scollo, and C. A. Vissers, eds.

Lloyd, J. W. 1987. Foundations of Logic Programming, 2nd Ed. Springer-Verlag, New York.

Milner, R. 1980. A calculus of communicating systems. Lecture Notes in Computer Science, vol. 92. Springer-Verlag, New York.

Nicollin, X., Richier, J.-L., Sifakis, J., AND Voiron, J. 1990. ATP: An algebra for timed processes. In Proceedings of the IFIP TC2 Working Conference on Programming Concepts and Methods (Sea of Gallilea, Israel), M. Broy and C. B. Jones, eds. pp. 402-429.

PARK, D. M. R. 1981. Concurrency and automata on infinite sequences. In Proceedings of the 5th GI Conference, P. Deussen, ed. Lecture Notes in Computer Science, vol. 104. Springer-Verlag, New York, pp. 167-183.

PlotkIn, G. D. 1981. A structural approach to operational semantics. Report DAIMI FN-19. Computer Science Department, Aarhus Univ.

PNuel, A. 1985. Linear and branching structures in the semantics and logics of reactive systems. In Proceedings of the 12th ICALP (Nafplion). Lecture Notes in Computer Science, vol. 194. Springer-Verlag, New York, pp. 15-32.

PRZYMUSINSKI, T. C. 1987. On the declarative semantics of deductive databases and logic programs. In Proceedings of the Foundations of Deductive Databases and Logic Programming, Jack Minker, ed. Morgan-Kaufmann Publishers, Inc. (Los Altos, Calif.), pp. 193-219.

PRZYMUSINSKI, T. C. 1989. Every logic program has a natural stratification and an iterated least fixed point model. In Proceedings of the 8th ACM SIGACT-SIGMOD-SIGART Symposium on Principles of Database Systems (Philadelphia, Pa., Mar. 29-31). ACM, New York, pp. 11-21. 
ReIter, R. 1980. A logic for default reasoning. Artif. Int. 13, 81-132.

VAANDRAGER, F. W. 1989. Specificatie en verificatie van communicatieprotocollen met procesalgebra. Unpublished (in Dutch).

VAANDRAGER, F. W. 1990. Algebraic techniques for concurrency and their application. Ph.D. dissertation. Centrum voor Wiskunde en Informatica, Amsterdam, The Netherlands.

VERHOEF, C. 1994. A general conservative extension theorem in process algebra. In Proceedings of PRO-COMET '94. IFIP Transactions A-56. Elsevier, Amsterdam, The Netherlands.

VERHOEF, C. 1995. A congruence theorem for structured operational semantics with predicates and negative premises. Nord. J. Comput. 2, 2 274-302.

RECEIVED NOVEMBER 1991; REVISED MARCH 1995; ACCEPTED SEPTEMBER 1995 